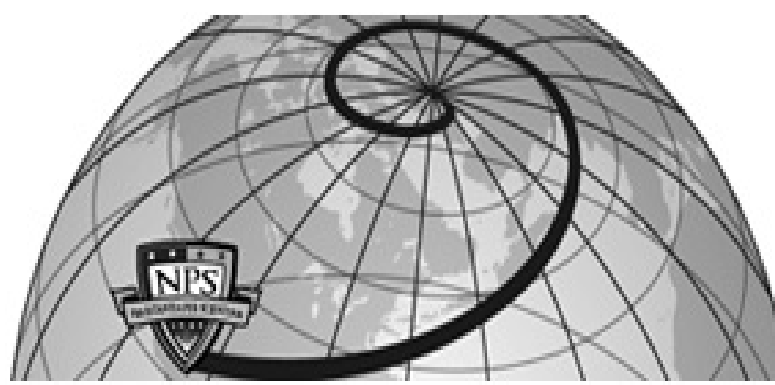

Calhoun: The NPS Institutional Archive DSpace Repository

Current issues in recrystallization: a review

Doherty, R.D.; Hughes, D.A.; Humphreys, F.J.; Jonas, J.J.; Jensen, Juul; Kassner, M.E.; King, W.E.; McNelley, T.R.; McQueen, H.J.; Rollett, A.D....

R.D. Doherty et al. : Materials Science and Engineering A238 (1997) 219274 http://hdl.handle.net/10945/40175

This publication is a work of the U.S. Government as defined in Title 17, United States Code, Section 101. Copyright protection is not available for this work in the United States.

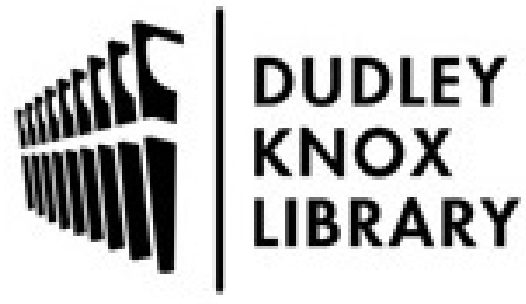

http://www.nps.edu/library
Calhoun is the Naval Postgraduate School's public access digital repository for research materials and institutional publications created by the NPS community. Calhoun is named for Professor of Mathematics Guy K. Calhoun, NPS's first appointed -- and published -- scholarly author.

Dudley Knox Library / Naval Postgraduate School 411 Dyer Road / 1 University Circle Monterey, California USA 93943 


\title{
Current issues in recrystallization: a review
}

\author{
R.D. Doherty ${ }^{a}$, D.A. Hughes ${ }^{\text {b }}$, F.J. Humphreys ${ }^{\text {c }}$, J.J. Jonas ${ }^{\text {d }}$, D. Juul Jensen ${ }^{\text {e }}$, \\ M.E. Kassner ${ }^{\text {f,* }}$, W.E. King ${ }^{\mathrm{g}}$, T.R. McNelley ${ }^{\mathrm{h}}$, H.J. McQueen ${ }^{\mathrm{i}}$, A.D. Rollett ${ }^{\mathrm{j}}$ \\ a Department of Materials Engineering, Drexel University, Philadelphia, PA 19104, USA \\ ${ }^{\mathrm{b}}$ Center for Materials and Applied Mechanics, Sandia National Laboratory, Livermore, CA 94550, USA \\ ${ }^{\mathrm{c}}$ Manchester Materials Science Center, UMIST and University of Manchester, Manchester M1 7H5, UK \\ ${ }^{\mathrm{d}}$ Department of Metallurgical Engineering, McGill University, Montreal, Quebec H3A 2A7, Canada

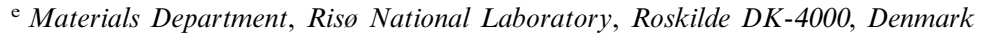 \\ ${ }^{\mathrm{f}}$ Department of Mechanical Engineering, Oregon State University, Covallis, OR 97331, USA \\ ${ }^{g}$ Chemistry and Materials Science Directorate, Lawrence Livermore National Laboratory, Livermore, CA 94550, USA \\ ${ }^{\mathrm{h}}$ Department of Mechanical Engineering, Naval Postgraduate School, Monterey, CA 93943, USA \\ ${ }^{i}$ Department of Mechanical Engineering, Concordia University, Montreal 63G 1M8, Canada \\ ${ }^{\mathrm{j}}$ Department of Materials Science and Engineering, Carnegie-Mellon University, Pittsburgh, PA 15213, USA
}

Received 11 April 1997

\begin{abstract}
The current understanding of the fundamentals of recrystallization is summarized. This includes understanding the as-deformed state. Several aspects of recrystallization are described: nucleation and growth, the development of misorientation during deformation, continuous, dynamic, and geometric dynamic recrystallization, particle effects, and texture. This article is authored by the leading experts in these areas. The subjects are discussed individually and recommendations for further study are listed in the final section. (C) 1997 Elsevier Science S.A.
\end{abstract}

Keywords: As-deformed state; Nucleation; Recrystallization

\section{Introduction}

The objectives of this article are two-fold. First, the current understanding of the fundamentals of recrystallization is summarized. This includes understanding the cold and hot-deformed state. Next, with the state of the art established, recommendations for future research are made. Several aspects of recrystallization are described. The authors of this paper are the contributors to each aspect described in a separate section. These are listed below with the authors of each section identified. Overall editing was performed by external reviewers as well as the contributors.

1. Introduction (R.D. Doherty and M.E. Kassner)

2. Theories of nucleation and growth during recrystallization (R.D. Doherty)

3. Formation of deformation induced high angle

* Corresponding author. Fax: + 1541 7372600; e-mail: kassner@engr.orst.edu boundaries and their effect on recrystallization (D.A. Hughes and D. Juul Jensen)

4. Issues in texture development and simulation of recrystallization (A.D. Rollett)

5. Second phase particles and recrystallization (F.J. Humphreys)

6. Conventional dynamic recrystallization (DRX) (J.J. Jonas)

7. Continuous reactions (T.R. McNelley)

8. Geometric Dynamic recrystallization (M.E. Kassner)

9. The hot worked state (H.J. McQueen)

10. The role of grain boundaries in recrystallization (W.E. King)

11. Recommendations for further study (all authors)

It is, of course, useful to carefully define the term 'recrystallization'. The authors have agreed that recrystallization is the formation of a new grain structure in a deformed material by the formation and migration of high angle grain boundaries driven by the stored energy 
of deformation. High angle grain boundaries are those with greater than a $10-15^{\circ}$ misorientation. Recovery can be defined as all annealing processes occurring in deformed materials that occur without the migration of a high angle grain boundary. Grain coarsening can, in turn, be defined as processes involving the migration of grain boundaries when the driving force for migration is solely the reduction of the grain boundary area itself. These definitions are consistent with some earlier definitions [1].

\section{Theories of nucleation and growth during recrystallization}

\subsection{Introduction}

The theories and recent experimental insights into the processes of nucleation and growth are reviewed with emphasis on what is not yet fully understood. In the light of these views, a range of needed new theoretical and experimental studies is proposed to improve the understanding and modelling of recrystallization mechanisms.

The process of recrystallization of plastically deformed metals and alloys is of central importance in the processing of metallic alloys for two main reasons. The first is to soften and restore the ductility of material hardened by low temperature deformation (that occurring below about $50 \%$ of the absolute melting temperature, $0.5 T_{\mathrm{m}}$ ). The second is to control the grain structure of the final product. In metals, such as iron, titanium, and cobalt that undergo a phase change on cooling, the grain structure is readily modified by control of the phase transformation. For all other metallic alloys, especially those based on copper, nickel, and aluminum, recrystallization after deformation is the only method for producing a completely new grain structure with a modified grain size, shape, and, in particular, mean orientation or texture. The subject has been recently given a long overdue review in the monograph by Humphreys and Hatherly [2] that nicely complements the much earlier multi-authored volume edited by Haessner [3]. This section aims to summarize the current status of the still rather limited scientific understanding of the two central processes of recrystallization - nucleation and growth of new grains - with the objective of focusing on what, in the authors' opinion, seems to be the necessary new studies for improved scientific understanding of the process. Although there is a great deal of empirical knowledge of the microstructures that can be produced during current industrial processing, the ability to produce more nearly ideal microstructures for different applications is very limited and it is in order to gain improved control of recrystallization processing that increased scientific understanding is needed.
During deformation energy is stored in the material mainly in the form of dislocations. This energy is released in three main processes, those of recovery, recrystallization, and grain coarsening. The usual definition of recrystallization [1] is the formation and migration of high angle grain boundaries driven by the stored energy of definition. On this definition recovery includes all processes releasing stored energy that do not require the movement of a high angle grain boundary. Typically, recovery processes involve the rearrangement of dislocations to lower their energy, for example by the formation of low-angle subgrain boundaries. Grain coarsening is the growth of the mean grain size driven by the reduction in grain boundary area [4,5]. Coarsening can take place by either 'normal' grain growth, whose main mechanism is the disappearance of the smallest grains in the distribution, or 'abnormal' grain growth. The latter process involves the growth of a few grains which become much larger than the average.

\subsection{Discussion}

\subsubsection{Nucleation and growth in recrystallization}

In all structural transformations there are two alternative types of transformation as originally recognized by Gibbs, see for example Doherty [6]. In the first of these, Gibbs I, typically called 'nucleation and growth', the transformation is extensive in the magnitude of the structural change but is, initially, spatially localized with a sharp interface between the old and new structures. The second type of transformation, Gibbs II, often described as 'continuous' or 'homogeneous' (the best known example being spinodal decomposition), the transformation is initially small in the magnitude of the structural change, but it occurs throughout the parent structure. In the range of processes seen on annealing plastically deformed materials, both dislocation recovery, that takes place before and during recrystallization and also normal grain growth are clearly Gibbs II transformations which occur uniformly throughout the sample while recrystallization and abnormal grain growth are Gibbs I transformations - at least on the observational length scales of about $1-5 \mu \mathrm{m}$ for recrystallization or about $0.1-1 \mathrm{~mm}$ for abnormal grain growth [2]. At these length scales, typically studied by optical microscopy, the new recrystallized grain or the abnormally large grains, are seen to be growing into the prior structure with a sharp interface, a grain boundary, as the 'recrystallization front' between the deformed and new grains (see Fig. 1). The usual name of 'nucleation and growth' for a Gibbs I transformation is based on the two apparently distinct steps in the process: (i) the initial formation of the new grain; and (ii) its growth. 
In the scientific study of phase transformations, one kinetic model of nucleation has been dominant. This is the thermal fluctuation model initially developed in physical chemistry [8,9] but applied very successfully to solidification and then to solid state phase transitions by Turnbull $[10,11]$ as described very fully in the review by Christian [12] and recently updated [6]. If there is a volume free energy driving pressure of $\Delta G_{\mathrm{v}}$ (in units of $\mathrm{Jm}^{-3}$ or $\mathrm{Pa}$ ), an interfacial energy of $\gamma$ (in units of $\mathrm{Jm}^{-2}$ ) between the old and new structures and, for heterogeneous nucleation of a defect interface, a contact angle $\theta$, there is an energy barrier, $\Delta G^{*}$, to the formation of a critically sized new region (usually called 'embryos') that are just stable and capable of growth. $\Delta G^{*}$ is given by Eq. (1):

$\Delta G^{*}=\left\{\alpha \gamma^{3} / \Delta G_{\mathrm{v}}^{2}\right\} f(\cos \theta)$

$\alpha$ is a number that varies with the shape of the new region, for example $\alpha$ is $16 \pi / 3$ for a spherical nucleus, and the function $f(\cos \theta)$, that is typically between 0.1

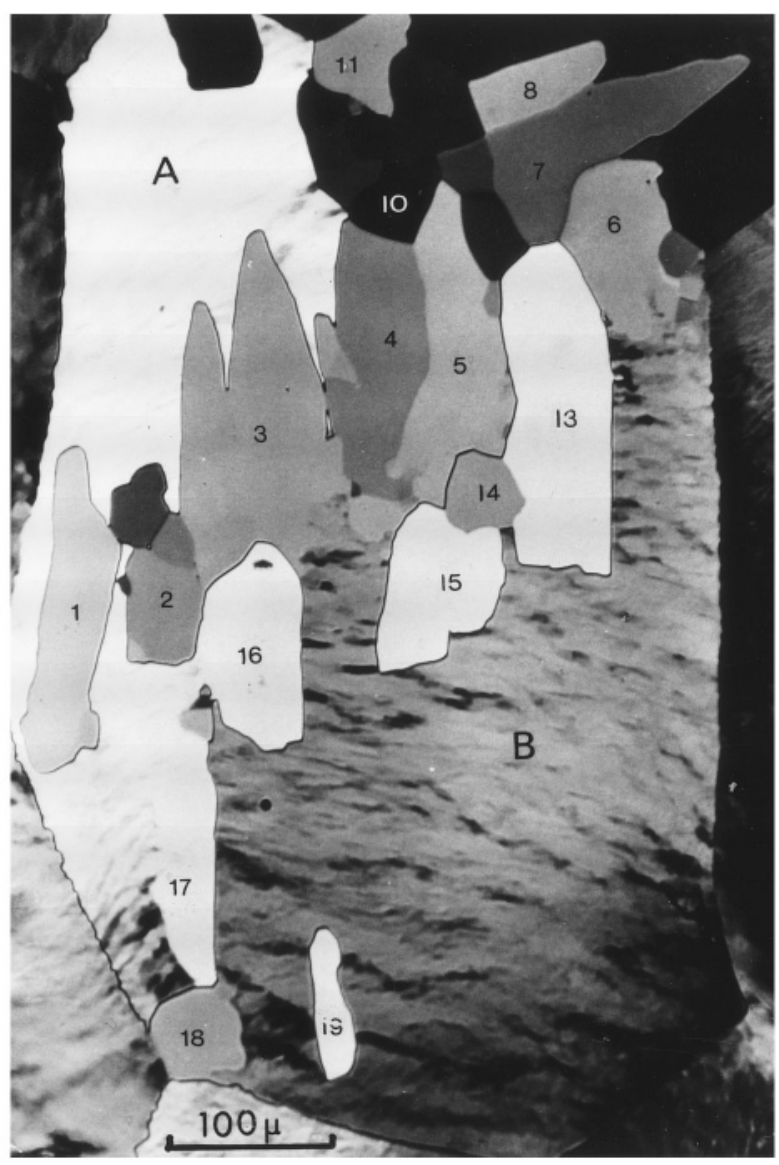

Fig. 1. Optical micrograph of partially recrystallized coarse grained aluminum compressed $40 \%$. The large grain has fragmented into two misoriented regions, $\mathrm{A}$ and $\mathrm{B}$, misoriented by about $40^{\circ}$. New grains, 13-17, and 19 have an A orientation and are growing into $\mathrm{B} ; 1-8$, 11 , and 18 have a B orientation and are growing into A. As in all examples of recrystallization, the inhomogeneous nature of the process is clear. Bellier and Doherty [7] courtesy of Acta Metallurgica. and 0.5 , depending on the geometry of the defect [12]. In a matrix containing $N_{\mathrm{v}}$ atoms per unit volume, the density of critical embryos, $n_{\mathrm{v}}^{*}$, is given by Eq. (2):

$n_{\mathrm{v}}^{*}=N_{\mathrm{v}} \exp \left(-\Delta G^{*} / k T\right)$

and the rate of formation of new grains, $I_{\mathrm{v}}\left(\mathrm{m}^{-3} \mathrm{~s}^{-1}\right)$ is:

$I_{\mathrm{v}}=\beta n_{\mathrm{v}}^{*}$

The kinetic parameter $\beta$ involves various terms that include the rate of atom addition to the embryo (proportional to the interface mobility) and the reduction in the equilibrium value of $n_{\mathrm{v}}^{*}$, due to the loss of embryos as they evolve into growing new particles.

As reviewed recently [6], the predictions of the kinetic theory are found to be in excellent qualitative agreement with a vast range of experimental behavior and, in a few cases, for example, for homogeneous nucleation in solidification and homogeneous precipitation reactions with a low energy fully coherent interface between phases of very similar structure (GP zones in $\mathrm{Cu}-\mathrm{Co}$ and ordered $\gamma^{\prime}$ precipitates, $\mathrm{Ni}_{3} \mathrm{Al}$, in $\mathrm{Ni}-\mathrm{Al}$ ), quantitative agreement as well. A major problem for the study of nucleation in recrystallization is that it is easily shown, for example [13], that given the typically low values of the stored energy of deformation, $\Delta G_{\mathrm{v}} \approx 0.1-$ $1 \mathrm{MPa}$ [2] and the high value of the energy of a high angle grain boundary, $\gamma \approx 0.5 \mathrm{Jm}^{-2}$ [2] that $\Delta G^{*}$ is so large, of order $10^{8} \mathrm{kT}$, that new grains cannot form by the mechanism of thermal fluctuation even at temperatures $\left(T>0.5 T_{\mathrm{m}}\right)$ where atomic and grain boundary mobility are significant and where grains do indeed 'nucleate' and grow. That is, the observed rate of formation of new grains is found to be almost infinitely larger, by some impossibly large factor such as $10^{50}$ times, than the nucleation rate predicted by the thermal fluctuation model, Eq. (3).

As a result of this disagreement, it is now universally recognized [2,14] that, as first proposed by Cahn [15] in 1949, the new grains do not 'nucleate' as totally new grains by the atom by atom construction assumed in the kinetic model. What happens is that these new grains grow from small regions, recovered subgrains or cells, that are already present in the deformed microstructure. One of the many important consequences of this idea is that the orientation of each new grain arises from the same orientation present in the deformed state [2]. This results has been experimentally confirmed many times, see for example [14]. As discussed, for example, by Hatherly [16] and clearly demonstrated by Haasen [17], new orientations can develop in low stacking fault energy materials, that form annealing twins, by growth twinning of a growing new grain. In these cases, however, the original orientation in the deformed state can be tracked back from the resulting first or, in some cases, in thin transmission 
electron microscopy (TEM) foils [17], higher order twins. The critical difference between nucleation in recrystallization and in the other types of structural transformation such as solidification or the precipitation of second solid phase, $\mathrm{P}$, from a supersaturated matrix phase, $M$, is that, in these latter cases the required atomic arrangements characteristic of the new structures do not exist in the parent structure (liquid or M) and so must be built up atom by atom to the critical size. This will take place at the rate given by Eq. (3). In a deformed metal, for example heavily rolled aluminum, copper, or brass, even though there is a very high defect density, the equilibrium fcc arrangement of atoms is still present everywhere. The diffraction pattern remains that of the fcc metallic structure in all deformed fcc metals, though the diffraction peaks are broadened. Strain broadened X-ray diffraction lines is heavily cold rolled aluminum alloys with very high strain hardening promoted by various solutes has been reported recently [18]. In abnormal grain growth, the equivalent situation occurs. The reaction involves a very small minority of the existing grains starting to grow at the expense of the vast majority of the other grains, which do not grow at any significant rate [2]. Here again the special grains do not have to form; they, like the embryos of the new grains in recrystallization, are present in the starting structure. The question in both cases, recrystallization and abnormal grain growth, is how are the successful embryos or special grains selected? The present review will address only the case of the subgrain selection in successful regions in recrystallization, although, in the opinion of the author, the two topics have much in common, at least as regards the problem of 'nucleation'.

\subsubsection{Grain boundary energy and mobility}

Following the suggestion of Cahn [15] that nuclei grew from deformed subgrains, the question arose over 40 years ago of why only a very small minority of subgrains made this transition. A simple calculation $[13,14]$ indicates the magnitude of the problem. Moderately deformed, polycystalline aluminum develops a subgrain size of about $1 \mu \mathrm{m}$ but after complete recrystallization the sample can evolve to a grain size of about $100 \mu \mathrm{m}$, see, for example, recrystallization of moderately deformed aluminum [9]. An increase in diameter of about 100 indicates a volume increase, from the subgrain embryo to the final recrystallized grain, of about $10^{6}$. This estimate indicates that only about one subgrain in a million becomes a successful recrystallization nucleus in moderately deformed aluminum. Cottrell [19] suggested that a critical reason for this small probability of success was the low mobility of most subgrain boundaries since most of the subgrains have only a small misorientation with their neighbors.
Only subgrains with a high misorientation angle to the adjacent deformed material appear to have the necessary mobility to evolve into new recrystallized grains. This old idea is completely supported by extensive experimental evidence that 'nucleation' only takes place at regions in the microstructure with high local misorientation. Evidence for this was reviewed in 1978 [14] and subsequent studies strongly confirm this conclusion [2]. Typical nucleation sites, all of which have high local misorientations, include:

1. pre-existing high angle grain boundaries;

2. misoriented 'transition' bands inside grains between different parts of the grain that have undergone different lattice rotations due to different slip systems being activated (Fig. 1 shows an example of a misoriented transition band between regions $\mathrm{A}$ and $\mathrm{B}$ at which nucleation of new grains has occurred [7]);

3. at highly misoriented deformation zones around large particles;

4. within highly misoriented regions within shear bands (these are bands of highly localized deformation seen in materials with high stored energies); and

5. at many places within very heavily deformed materials $(\varepsilon>3-5)$, such as highly drawn wires.

Humphreys and Hatherly [2] recently reviewed the surprisingly limited studies of the orientation dependence of grain boundary mobility in metals and concluded that there were indeed very large mobility differences of $100-1000$ times, directly measured, between low angle $\left(2-5^{\circ}\right)$ and high angle $\left(>15^{\circ}\right)$ grain boundaries. In high purity copper, the low angle boundaries showed activation energies close to that of bulk diffusion (204 $\mathrm{kJ} \mathrm{mol}^{-1}$ ) while the high angle boundaries had the lower activation energies of boundary diffusion (125 kJ mol-1) (see Fig. 2). In low-angle, dislocation, boundaries, the rate determining step appears to be vacancy diffusion between dislocations, in near perfect crystal, while in high angle grain boundaries the rate determining step appears to be the atom transport by single atom jumps from the shrinking to the growing grains in the defect structure of a high angle grain boundary. Very recently Ferry and Humphreys [20] produced direct evidence for the increase of mobility in $\mathrm{Al}-0.05 \% \mathrm{Si}$ subgrain boundaries of about 14 times as the misorientation increased from 2 to $5^{\circ}$ and 2500 times from $2^{\circ}$ subgrain boundaries to high angle recrystallization boundaries on annealing at $300^{\circ} \mathrm{C}$. Fig. 1 shows examples of this effect. New grains 3 and 17 are only growing into the deformed regions A and $\mathrm{B}$, respectively, with which they are strongly misoriented and not into the regions with which they share a common orientation; 17 has a low angle misorientations with $\mathrm{A}$ and 3 with $\mathrm{B}$ [7]. 


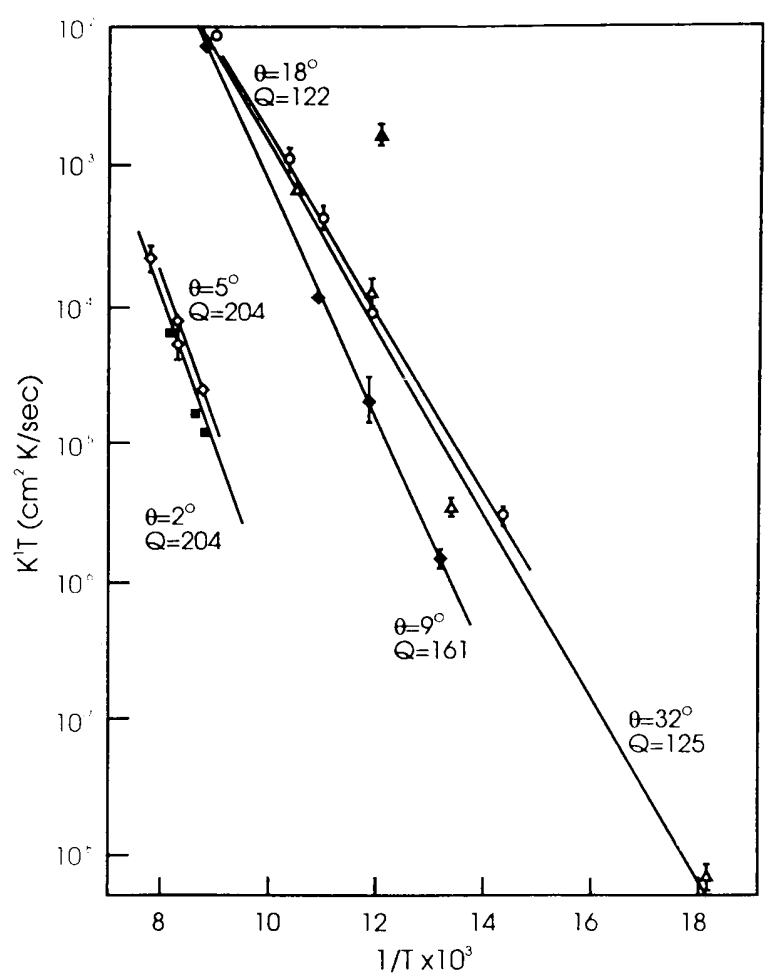

Fig. 2. The much lower mobility $\left(K^{\prime}\right)$ and higher activation energy of low angle grain boundaries in high purity copper. From Humphreys and Hatherley [2] derived from the results of Viswanathan and Bauer.

Humphreys and Hatherly [2] have also reviewed other topics of importance to the consideration of grain boundary mobility. These topics include the important effect of solute drag and the possible role of grain boundary structure. For solute drag, the main effects seem quite well established in that solute, especially that with low very limited solubility in the metal, strongly adsorbs at grain boundary and acts to inhibit boundary motion. The effect is most noticeable at very low solute levels and one of its most dramatic influences on recrystallization can be seen in ultra high purity aluminum which, when deformed at low temperatures, can readily recrystallize at or below room temperature $\left(0.3 T_{\mathrm{m}}\right)$, see for example Haessner and Schmidt [21], while with a more typical purity even as little as $0.01 \mathrm{wt} \% \mathrm{Fe}$ in solution, the recrystallization temperature is very much higher at $250^{\circ} \mathrm{C}\left(0.6 T_{\mathrm{m}}\right)$ [7]. The activation energy of recrystallization, involving the movement of high angle grain boundaries in the presence of solute, rises from that expected for grain boundary diffusion (as seen for very high purity material) to that of bulk diffusion of either the solvent or the solute in metals of even moderate impurity levels. The increase of activation energy in the presence of solute raises the question of by how much the higher mobility of high angle versus low angle boundaries discussed above might be affected by solute. The recent demonstration by Ferry and Humphreys
[20] for the retention of mobility differences of 1000 even in the presence of $0.05 \% \mathrm{Si}$ in $\mathrm{Al}$ is reassuring as it indicates the large difference in boundary mobilities, previously found in very high purity copper, are quantitatively as well as qualitatively similar to the effects observed in commercially pure materials.

There is, in addition, the much discussed question of the relative mobility of a few special high angle grain boundaries, some of which are close to so called coincident site boundaries. An important example is $\Sigma 7$ (that is with one atom in seven coincident in position in both grains) the $38^{\circ}$ misoriented boundary with a common $\langle 111\rangle$ rotation axis. The higher mobility of this boundary is central to one model of the 'oriented growth' of fcc recrystallization texture [2]. It is clear that, at least for the tilt boundaries of $\Sigma 7$, boundaries parallel to the $\langle 111\rangle$ axis do have higher mobility than average high angle boundaries, at least in the presence of solute, but this appears to be offset by the significantly lower mobility of twist boundaries [22]. A nice demonstration of this provided by Ardakani and Humphreys [23] who found the new grains with a near $40^{\circ}\langle 111\rangle$ misorientation relationship with the matrix in deformed single crystals of $\mathrm{Al}-0.05 \% \mathrm{Si}$ grew ten times faster in the tilt than in the twist direction. (The tilt boundary is one with the common $\langle 111\rangle$ rotation axis lying parallel to the boundary plane; the twist boundary has the axis normal to the plane).

\subsubsection{Transition from subgrain embryo to growing new grain}

It has been recognized for many years [24] that for a subgrain to make this transition, possession of a high angle misorientation is a necessary but not sufficient criterion. The subgrain, to become a successful new grain, must have, in addition, an energy advantage, usually a larger size in order to be able to grow rather than shrink and vanish. The need for both a size advantage and a high local misorientation appears to be a reasonable explanation of the rarity of the process, discussed above. In an earlier review [14], it was noted that the deformation process itself may, in some cases, give a favored subgrain both advantages, high local misorientation and a size advantage simultaneously. The original model of Beck and Sperry [25] for 'strain induced grain boundary motion' in which large subgrains on one side of a grain boundary can immediately grow into the matching grain is a clear example of this effect. Experimental evidence for this suggestion, from orientation dependent stored energy, for example in heavily cold rolled iron [26], is well established. However, in other cases, for example in moderately compressed aluminum [7,27], there is often no significant orientation dependent subgrain size differences produced directly by deformation. In this latter case, it was found that the necessary size advantage of the sub- 
grains adjacent to high misorientations had to be developed by a second, thermally activated process subsequent to the deformation.

In the case of moderately deformed aluminum it was found [27-29] that the second process was that of subgrain coalescence. The coalescence of several subgrains on one side of a grain boundary was observed to yield a subgrain large enough to grow rapidly into the adjacent grain [27,28]. This coalescence process was highly localized; it only occurred at isolated points on the grain boundaries in aluminum, where misoriented 'transition bands', in the grain in which coalescence occurred, reached the grain boundary [27]. A recovery model based on the climb and cross slip of dislocation loops that could successfully account for the location and kinetics of the coalescence was subsequently proposed and tested [29]. In work currently in progress, Woldt (E. Woldt, private communication, 1996). is directly reporting subgrain coalescence as occurring at the grain boundaries in heavily cold rolled high purity copper. Here the process gives a measured energy release preceding recrystallization [30] and so in this case it appears to be occurring more generally than in the more moderately deformed aluminum [27-29]. The development of new grains by thermal recovery of the 'deformation zone' around large particles in cold deformed metals as 'particle stimulated' nucleation [2] is a further example of a clear two-step nucleation process. The first step occurs in deformation when misorientation develops in the deformation zone but with small subgrains in the zone. The second occurs on annealing when subgrain growth occurs within the deformation zone giving the misoriented region the necessary size advantage.

A more recent example of the difference between a microstructure in which the necessary conditions of size and misorientation were produced by deformation alone and where a post-deformation anneal was required to produce this microstructure has been reported by Samajdar [31]. He studied the deformed microstructure in a commercial purity aluminum that had been plane strain extruded to two strains, 84 and $96 \%$ reduction, at $320^{\circ} \mathrm{C}$. It was found that a portion of each pre-existing near 'cube' grain, $\{100\}\langle 100\rangle$, retained its near cube orientation during deformation and, on quenching from the extrusion press, the cube oriented material had significantly larger subgrain sizes (and smaller subgrain misorientations) than were seen in the adjacent material, which was separated from the deformed cube regions by a sharp, high angle grain boundary. In the case of the highest reduction, the 'deformed cube bands' were only one to two subgrains thick, so the large subgrains were in direct contact with the high angle grain boundary. As a result, recrystallization started immediately on annealing and, in fact, a small amount of recrystallization growth of the cube embryos had begun in the material after quenching from the extrusion press. However, the material with the smaller extrusion reduction had deformed cube bands that were about eight to ten subgrains thick. The largest subgrains, which were seen to have the smallest deviations from the exact cube orientation, were found in the center of the cube band with smaller, and more misoriented from exact cube, subgrains between themselves and the high angle grain boundary. On annealing, the latter material at a temperature in which recrystallization went to completion in $250 \mathrm{~s}$, there was an incubation period of $50 \mathrm{~s}$ before any detectable recrystallization occurred. During this period there was a slow growth of the large subgrains in the center of the deformed cube band to the edge of the band before the much more rapid 'recrystallization' growth occurred of the cube regions into the misoriented material of the adjacent deformed band.

\subsubsection{Formation (nucleation) and growth in recrystallization texture development}

A subject that has been a major dispute for over 50 years has been the origin of the strong recrystallization texture often found after heavy deformation. In many cases, after only moderate deformation, nearly random textures are produced. On annealing after very heavy reductions, a strong recrystallization texture is usually found, which may involve the partial retention of the deformation texture but quite often a very different but very strong new texture forms. A classic example is the formation of a very strong cube texture in some (but not all) heavily rolled fcc metals [31]. The cube orientation is a finite but very small part of the deformation texture. Two major alternative models exist for the formation of a strong new texture-usually described as 'oriented nucleation' or 'oriented growth' $[1,31]$.

Oriented nucleation is the hypothesis that grains, with an orientation that dominates the fully recrystallized texture, nucleate more frequently than do grains of all other orientations. To describe this quantitatively, for example for the most discussed case of the formation of 'cube' texture after the recrystallization of heavily rolled fcc metals such as $\mathrm{Cu}$ or $\mathrm{Al}$, the fraction of grains, by number, within a selected misorientation, say 10 or $15^{\circ}$ from exact cube, $\alpha_{c}$, must be normalized by the fraction expected in a random grain structure, $\alpha_{\mathrm{r}}$ [32]. The condition for a strong 'oriented nucleation' effect is that:

$\alpha=\alpha_{\mathrm{c}} / \alpha_{\mathrm{r}} \gg 1$

That is, the frequency of the formation or birth of the new cube grains is much higher than the expected random frequency, so many of the grains will have the special orientation. The oriented growth factor, $\beta$, is determined by the relative sizes $\bar{d}_{\mathrm{c}} / \bar{d}_{\mathrm{r}}$ of the cube to the average grains [32]. That is, there is a strong oriented growth effect if: 
$\beta=\bar{d}_{\mathrm{c}} / \bar{d}_{\mathrm{r}} \gg 1$

In the opinion of the present author, it would be preferable to rename these two alternative models of oriented nucleation and oriented growth as: (i) the grain frequency effect; and (ii) the grain size effect. The reason for this suggested change is twofold. First, there is the confusion about the meaning of nucleation, which as discussed above for recrystallization, involves only the growth of a particular subgrain. Second, the terms oriented nucleation and oriented growth are often taken to indicate specific mechanisms for the frequency or size advantage. The use of the neutral terms frequency and size effect avoids these problems. The two measurable parameters, $\alpha$ and $\beta$, can describe the phenomenon of the recrystallization texture quantitatively but leave for subsequent studies the determination of the mechanisms giving rise to the effects, see for example [21].

An example of the first problem is illustrated by the review by Hatherly [16] who concluded from the idea that 'the only orientations that are available to a nucleus are those present in the deformed material' that, therefore, 'an oriented nucleation theory could not exist'. This argument is only valid if nucleation is restricted to the formation of a new crystal. However, the observation of $\alpha \gg 1$ is a real effect that needs a description and an analysis. An example of the second problem is the common observation that grain boundaries close to the $\Sigma 7$ orientation in fcc metals do, at least for certain boundary plane orientations, grow faster than other boundaries. This is clearly important in some single crystal experiments in which a lightly deformed single crystal is given many new grains by localized deformation, for example at one end of a rod, and all the new grains then compete with each other by growth in one direction along the rod [1]. In this case, any small growth advantage of a particular boundary will lead to a dominance of one orientation. This is clearly a size advantage and its mechanistic origin is well established [1]. However, in cases of cube texture in rolled fcc metals, the possession of a near $\Sigma 7$ misorientation between the new cube grains and a major component of the deformation texture 'S'. $\{123\}\langle 63 \overline{4}\rangle$, may or may not be responsible for the larger size of cube grains sometimes seen in the recrystallized structure $[1,22,33,34]$. One interesting new suggestion for understanding how different grain sizes might occur $(\beta>1)$ comes from Juul Jensen's [34] hypothesis of 'orientation pinning', Fig. 3. This idea is that a recrystallizing grain growing in a very heavily deformed material can meet many regions of different orientation. A grain such as cube, in most cases, will meet very few regions of similar orientation with which is shares low misorientation low mobility boundaries. Other grains, especially with orientations within the deformation tex-

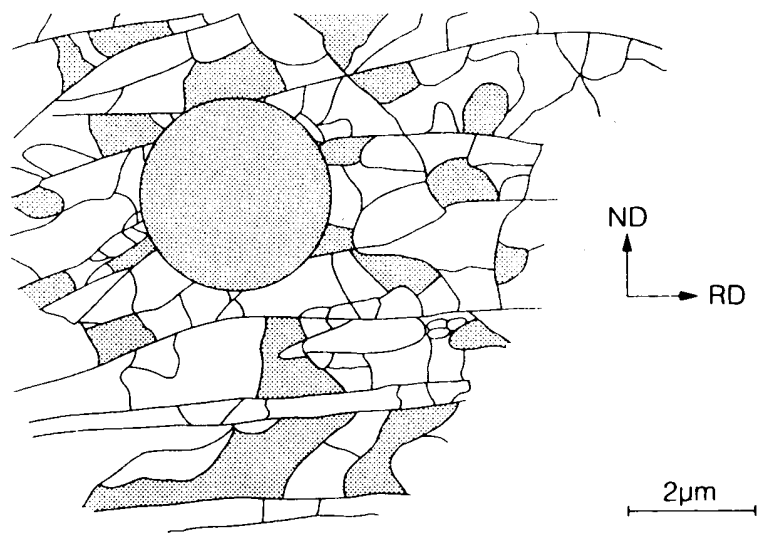

Fig. 3. Orientation pinning of a new grey circular grain when it meets regions of similar orientation (also grey) with which it has low angle, low mobility boundaries. After Juul Jensen [34].

ture, will meet many regions with similar orientations, and thus, on average, will have lower mobility and grow more slowly. Evidence supporting this idea of orientation pinning was presented where, for several examples of deformed aluminum, a value of $\beta>1$ for the cube grains was found [34].

A very similar idea to that of orientation pinning was put forward at the same meeting by Doherty et al. [35]. Their idea was called 'variant inhibition', Fig. 4. The only difference is the recognition based on experimental studies of warm plane strain extruded aluminum, is that the deformed bands of nearly constant orientation are stretched out in the extrusion direction, equivalent to the hot rolling direction, so that very strong inhibition of growth in the normal direction will occur. Grains from a deformation texture component will be inhibited from thickening by the low angle boundaries of the

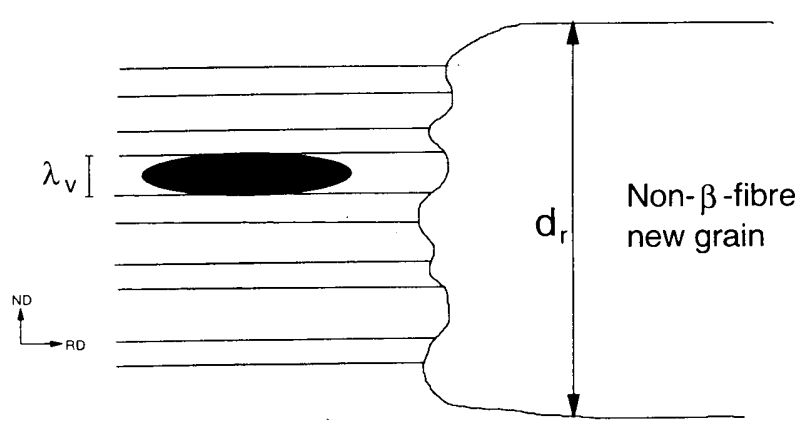

Fig. 4. Variant inhibition, the inhibited growth, in the normal direction, of a new grain belonging to one of the deformation texture components when it meets a thin deformed band of similarly oriented material. The spacing between the variants is $\lambda_{\mathrm{v}}$. The idea is identical to that of Fig. 3 except for presence of the banded structure drawn out in the rolling direction of warm rolled alloys. Such a thin grain could be easily destroyed by grain coarsening by the thicker, uninhibited grain whose orientation is found more rarely in the deformed matrix. After Doherty et al. [35]. 
same variant of the deformation texture component. This analysis identified two important effects apparently rising from the low mobility of low angle boundaries formed between a recrystallizing grain and deformed bands elongated in the rolling direction after heavy plane strain deformation. Each of these concerned values of the frequency parameter $\alpha$. In the first of these studies, low values of $\alpha$ were found for cube grains in heavily deformed aluminum alloy [35] despite having a high density of small initial cube grains that on the previously successful model, developed by Samajdar [31] from an idea of Duggan [36], should have had a very large value of $\alpha$. The recrystallized grains of size, $d_{\mathrm{R}}$, were much larger than the intercube band spacing $\lambda_{\mathrm{c}}$, given by:

$\lambda_{\mathrm{c}}=d_{0}\left(\alpha_{0} \exp \varepsilon\right)^{-1}$

$d_{0}$ and $\alpha_{0}$ are the mean grain size and cube grain frequency in the metal before deformation.

When $d_{\mathrm{R}} \approx \lambda_{\mathrm{c}}$ it was shown [31,36] that a very large value of $\alpha$ was found for the cube grains. However, when $d_{\mathrm{R}} \gg \lambda_{\mathrm{c}}$ the expected value of $\alpha$ was observed to fall [35]. To account for this failure, variant inhibition was proposed. That is, the closely spaced cube bands were suggested as hindering the formation (or the survival against grain growth) of new cube grains. Exactly similar arguments applied to deformation texture orientations which were able to account for the absence of any detectable frequency of grains with these 'retained rolling' orientation in the recrystallization texture of heavily warm plane strain deformed aluminum alloys.

\subsubsection{Role of the deformed microstructure on recrystallization}

It is clear from the ideas described above and from many previous reviews of the subject, for example $[2,14,35]$, that understanding recrystallization requires a detailed understanding of the deformed state. This requirement arises since the formation/nucleation of new grains is an instability of the deformed microstructure, depending on subgrain size heterogeneities present as potential embryos in the deformed state adjacent to high local misorientation. The growth of the new grains depends on both the mean stored energy [2] and on the frequency of new grains re-acquiring a low mobility boundary by meeting similar orientations in the deformed state $[35,36]$. At present, the microstructure must be fully characterized experimentally for each individual recrystallization study. When such a structural characterization has been carried out, for example $[7,20,24,27,31,36]$, the mechanisms of the birth and growth of new grains are usually rather easily understood, at least qualitatively. The investigation of the microstructure calls for detailed studied of the average texture, the size and misorientations of individual regions on a grain by grain basis, using initially TEM
$[24,26,27,37]$ and at an optical microscope or scanning electron microscope (SEM) level, Kossel X-ray diffraction [7], or more recently, and much more conveniently, by backscattered Kikuchi diffraction [22,31,34,35]. Such studies are very time consuming and, at present, specific to a given alloy after a given deformation. What is critically needed is an improved method predicting the detailed microstructures of deformed metals at the appropriate length scale, within and between grains [37,38] for a wide range of deformation conditions. The methods of finite element modelling $[39,40]$ do seem to be becoming, at least potentially, rather promising, though clearly much remains to be done in developing the method and applying it [38].

\section{Formation of deformation induced high angle boundaries and their effect on recrystallization}

\subsection{Introduction}

Microstructures and textures that develop during deformation set the stage for the changes that occur during recrystallization. This section considers the microstructural and microtextural development during cold to warm deformation and subsequent recrystallization. First, the general microstructural evolution during deformation is summarized and illustrated with some examples. The formation of deformation induced high angle boundaries within these structures is then considered since these boundaries have the high energy that is important for recrystallization. Lastly, the consequences of the deformed microstructure on the migration of annealing induced high angle boundaries during recrystallization (growth) are discussed and illustrated by specific examples.

The results presented are typical for standard deformation modes including uniaxial compression, channel die, plane strain compression tension, torsion, and rolling. The section emphasizes medium to high stacking fault energy (SFE) fcc metals including $\mathrm{Cu}, \mathrm{Ni}$ and $\mathrm{Al}$ and represents results on both single crystals and polycrystals.

\subsection{Deformation microstructures}

A comprehensive compilation and review of research on large strain deformation microstructures and textures prior to 1979 can be found in Ref. [41]. That review includes the important early work on dislocation boundaries and local orientations, for example $[24,42,43]$. In that early work it was found that deforming grains subdivide into misoriented regions and that high angle dislocation boundaries form during deformation. High angle boundaries are defined as boundaries with misorientations greater than $15-20^{\circ}$ 
[7]. Complementary to the next section, more recent and shorter reviews of the large strain state can be found in $[44,45]$. A sampling of individual current work on deformation microstructures is provided by the Proceedings of Low-Energy Dislocation Structures I, II and IV and ICOTOM 11 [46-49]. The significant developments in the more recent work on deformation microstructures include an evolutionary framework for grain subdivision [37], the ability to quantitatively characterize large numbers of individual crystallites and dislocation boundaries across grains with semi-automatic transmission electron microscopy techniques [50,51] and automatic EBSP (electron backscattering pattern) in the scanning electron microscope [52,53], and combinations of experimental observations with crystal plasticity simulations and modelling of dislocation boundaries [54-59].

\subsubsection{Grain subdivision}

Deformation microstructures are characterized by grain subdivision into differently oriented regions [37]. Dislocation boundaries separate the rotated regions at two size scales (and sometimes three for the case of special crystals) from small to large strains. Equiaxed dislocation cells comprise the smallest volume element. At the next larger size scale, long flat dislocation boundaries surround blocks of cells that are arranged three to five deep between boundaries and several in length (Fig. 5(a) and (b)). The long flat boundaries include single walled dense dislocation walls (DDWs) and double walled microbands (MB) at small to medium strains. At large strains the cell blocks become very flat and are sandwiched by lamellar dislocation boundaries (LBs) that have replaced the small strain DDW and MB structures. In contrast to the small strain cell blocks, the cell blocks at the large strains are usually one to two cells deep and several along their length (Fig. 6(a) and (b)). Strips of equiaxed subgrains are also observed at large strains.

The cells boundaries are classified as incidental dislocation boundaries (IDB) [60] which have low misorientation angles on average. The DDWs, MBs, LBs, and subgrain boundaries are classified into a type of dislocation boundary called geometrically necessary boundaries (GNBs) [60] that separate differently deforming regions. These long GNBs are arranged in parallel families and have special macroscopic orientations with respect to the deformation axis. Both GNBs and IDBs increase their average misorientation angle and decrease their spacing with increasing strain and stress. However, GNBs increase their misorientation angle and decrease their spacing at a much higher rate than IDBs do. These structures that were originally defined for cold deformation have also been observed for warm deformation $[61,62]$.
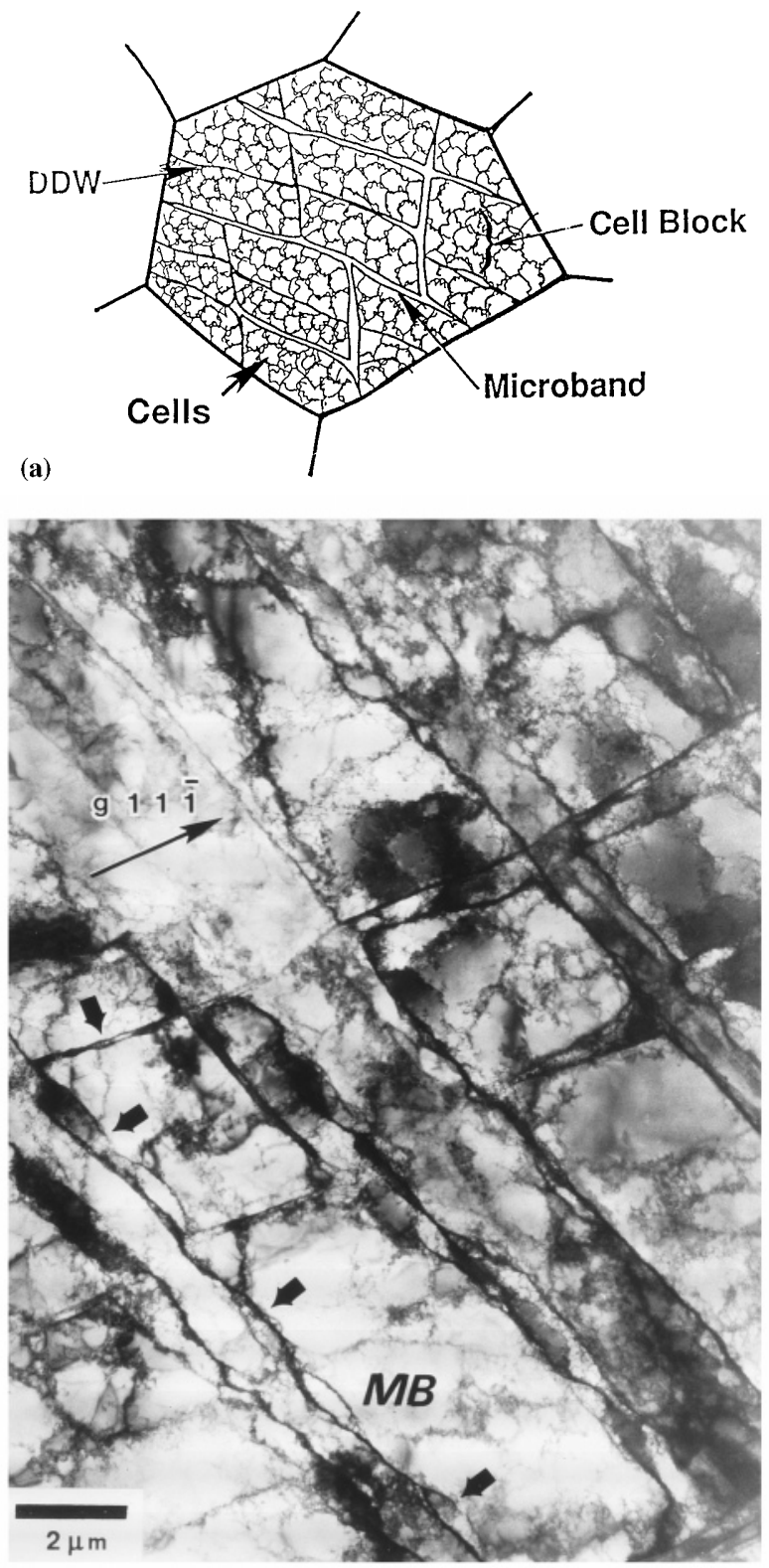

(b)

Fig. 5. Schematic (a) and TEM micrograph (b) of grain subdivision at small strain. Nickel deformed by torsion, $\varepsilon_{\mathrm{vM}}=3.5$. The direction of shear is shown by the arrows.

Because of the complexity of deformation structures, different dislocation boundaries have been classified according to a detailed consideration of boundary misorientation, morphology, spacing, crystallographic and macroscopic orientation with respect to the deformation axis. Definitions for these structures and boundary types have been given in [37] and a discussion of nomenclature in this field was held during a workshop on Fundamentals of Recrystallization in Zeltingen, Germany, and documented in a Scripta Mater. Conference set [63]. 
large strains
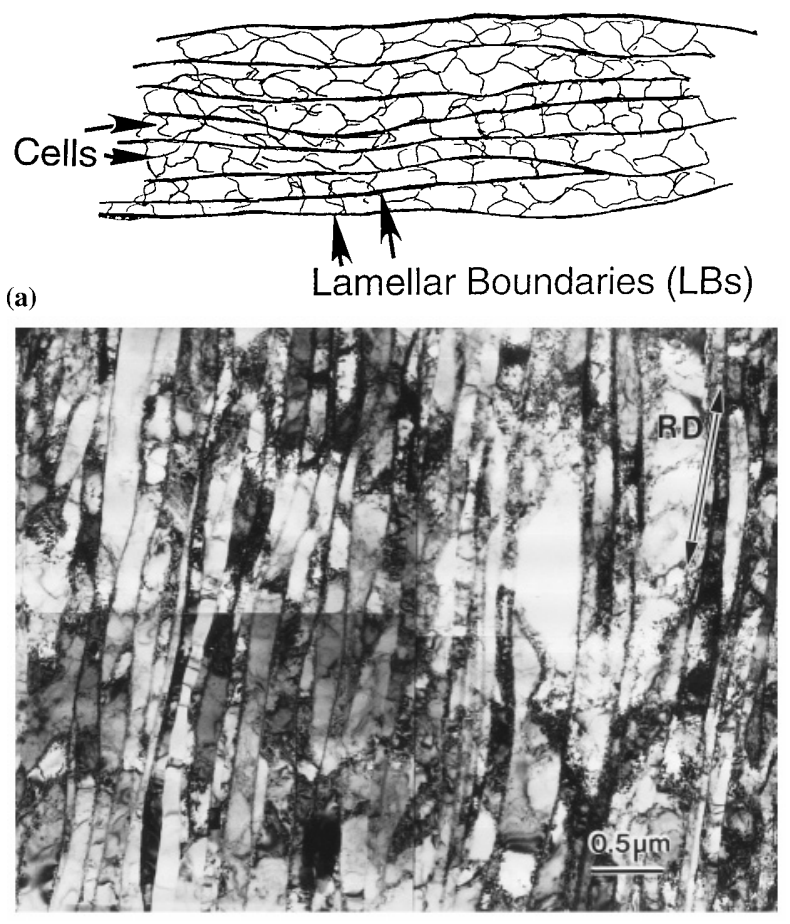

Fig. 6. Schematic (a) and TEM micrograph (b) of grain subdivision at large strain.

\subsubsection{Formation of deformation induced high angle boundaries}

A wide spread in misorientation angles is observed for GNBs and increases with increasing strain as a function of the average misorientation angle. Thus, at medium to large strains, some population of GNBs have increased their misorientation angle to the extent that they are classified as high angle boundaries. These high angle boundaries have formed through the normal cell block formation and associated dislocation processes. A second and equally important source of high angle boundaries occurs at intermediate strains and arises by the introduction of coarse slip in the form of S-bands [64]. Coarse localized slip in an individual S-band follows the crystallographic slip direction. However, localized groups of S-bands cluster into strip-like regions that have a macroscopic orientation with respect to the sample axis, e.g. parallel to the normal plane in rolling. Long high angle lamellar boundaries form at the boundaries between these clustered S-bands and matrix [64].

At the same time that these dislocation boundaries are forming, a preferred crystallographic texture is developing. Large crystal rotations occur as part of this texture evolution. During these large rotations, different parts of a grain may rotate to different end orienta-

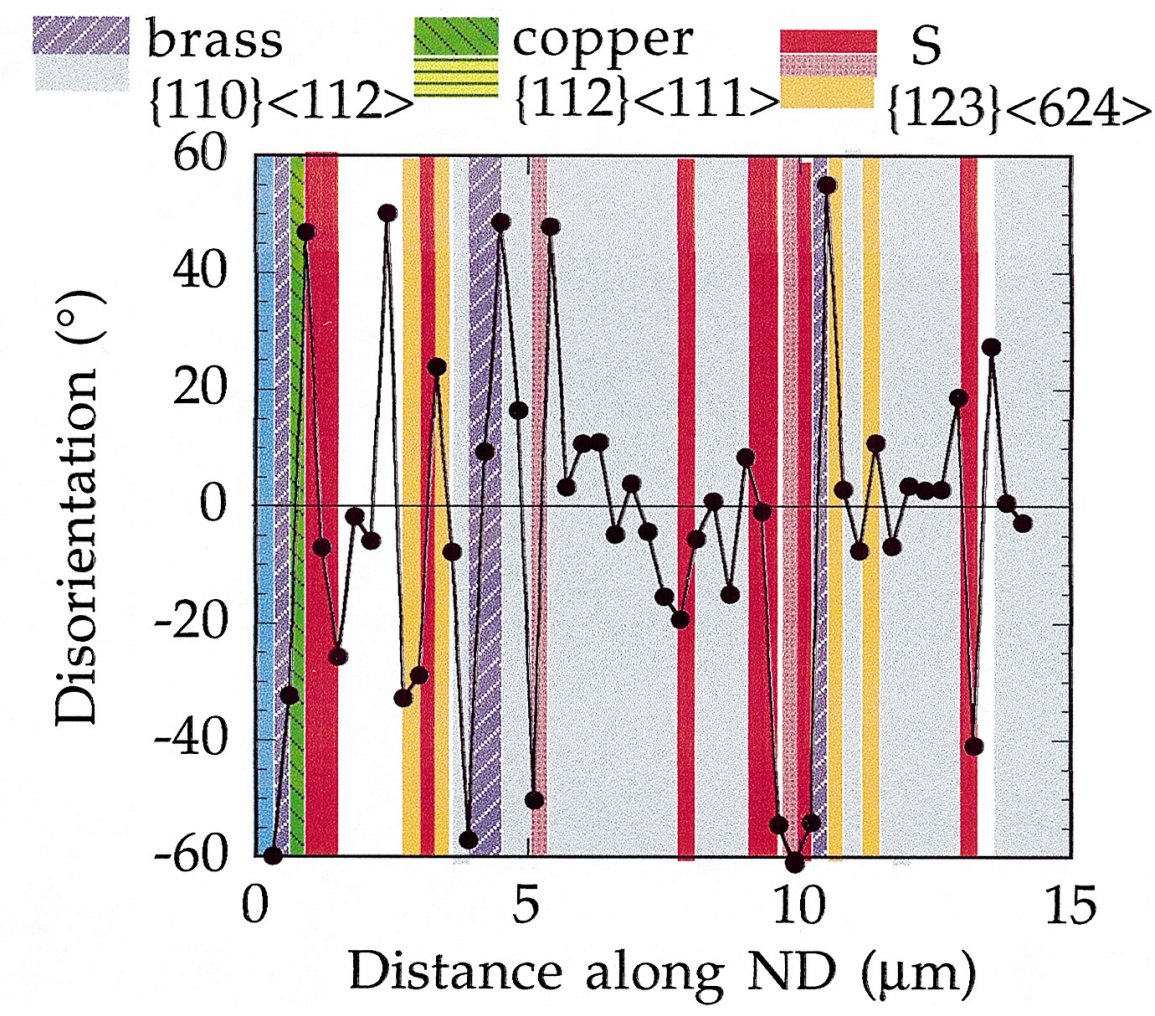

$90 \mathrm{cr}, \mathrm{c} 1,95$

Fig. 7. The disorientation angles measured across dislocation boundaries in the normal direction for aluminum $90 \%$ cold rolled show an alternating character with distance. These boundaries separate finely distributed texture components as shown by the color shading. 


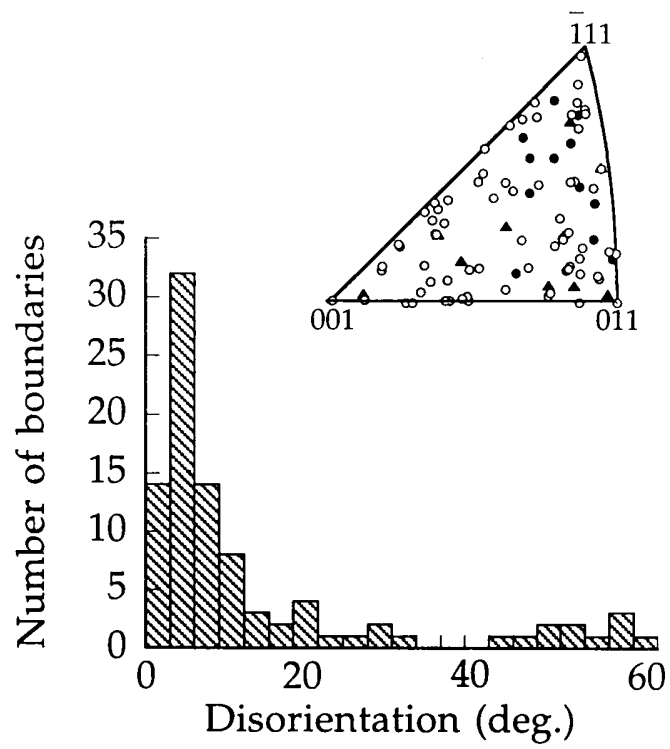

Fig. 8. Histograms showing the distribution of the magnitude of disorientations across dislocation boundaries measured along the normal direction (ND) of rolling in aluminum $90 \% \mathrm{cr}$. Note that the histograms generally showed two peaks in the distribution. The disorientation axes are plotted in standard triangles. Axes for $|\theta| \geq 35^{\circ}, \boldsymbol{\Delta} 18^{\circ} \leq|\theta|, \bullet|\theta|<18^{\circ}$.

tions due to the grain subdivision by dislocation boundaries that start at the beginning of deformation (see Ref. [65] for more details). Very high angle boundaries, e.g. $40-60^{\circ}$, consequently form during deformation due to this combination of dislocation and texture evolution. A plot of boundary disorientation (minimum misorientation based on crystal symmetry) versus distance for an 1180 type aluminum sample cr (cold rolled) $90 \%$ is shown in Fig. 7. These measurements were made using transmission electron microscopy and using Kikuchi pattern analysis. Note the large number of deformation induced high angle boundaries that are encountered in a short distance. Many small angle boundaries are also encountered. A histogram showing the range of both high and low misorientation angles following $90 \% \mathrm{cr}$ is shown in Fig. 8.

\subsubsection{Local orientations and orientation dependence}

The development of misoriented dislocation boundaries leads to a range of different crystal orientations throughout a grain, as shown by the colour shading in Fig. 7. The range of orientations within a grain increases from small differences at small strains to very large differences at large strains. These large differences go hand in hand with complex spatial patterns of orientations. There is also an orientation dependence on the microstructure development as illustrated by single crystal experiments. For example, crystals of brass $(\{100\}\langle 112\rangle)$ and Goss $(\{110\}\langle 001\rangle)$ orientations produce more homogeneous microstructures with lower misorientation angles compared with $\mathrm{Cu}(\{112\}\langle 111\rangle)$ oriented single crystals that develop heterogeneous microstructures and much larger misorientation angles; see Driver [66] for a recent summary. A moderately wide range of local orientations are expected to develop for the case of a general randomly oriented grain in a polycrystal.

Grain subdivision, depending on the grain orientation, can lead to heterogeneous distributions of stored energy and a wide distribution of misorientation angles across dislocation boundaries. At medium strain this subdivision can lead to the formation of deformation induced high angle boundaries which at large strain can have spacings an order of magnitude smaller than the spacings of the original grain boundaries.

\subsection{Growth during recrystallization}

Upon annealing, nuclei may form in the deformed microstructure. A viable nucleus by definition is surrounded, at least partly, by a high angle boundary which is able to migrate through the deformed microstructure causing he nucleus to grow (for a review of nucleation mechanisms, see Section 2 of this paper and $[2,67])$. The high angle boundary of the nucleus may or may not be related to a deformation induced high angle boundary that was already present in the deformation structure. The words deformation induced and annealing induced have been used in the text to differentiate between these different types of high angle boundaries, the former created during deformation and the latter during subsequent annealing and which surrounds a nuclei either in part or completely. Note that the following parts of this chapter refer primarily to the latter part: annealing induced high angle boundaries or boundary segments.

The driving force for migration of annealing induced boundaries is provided by the stored energy in the deformed matrix. The velocity of the migration, $v$, is generally regarded to be a product of the mobility term, $M$, and the driving force $\Delta f$ :

$v=M \cdot \Delta f$

For a discussion of the underling theory and experimental validation of Eq. (7) see [2,68].

The mobility, the driving force and, therefore, the velocity of migration depend on a whole range of materials, deformation and annealing parameters [2]. Important under most conditions is, however, the crystallographic orientation relationship across the annealing induced boundaries. Barrett [69] first suggested that the mobility of nuclei boundaries depends on such 


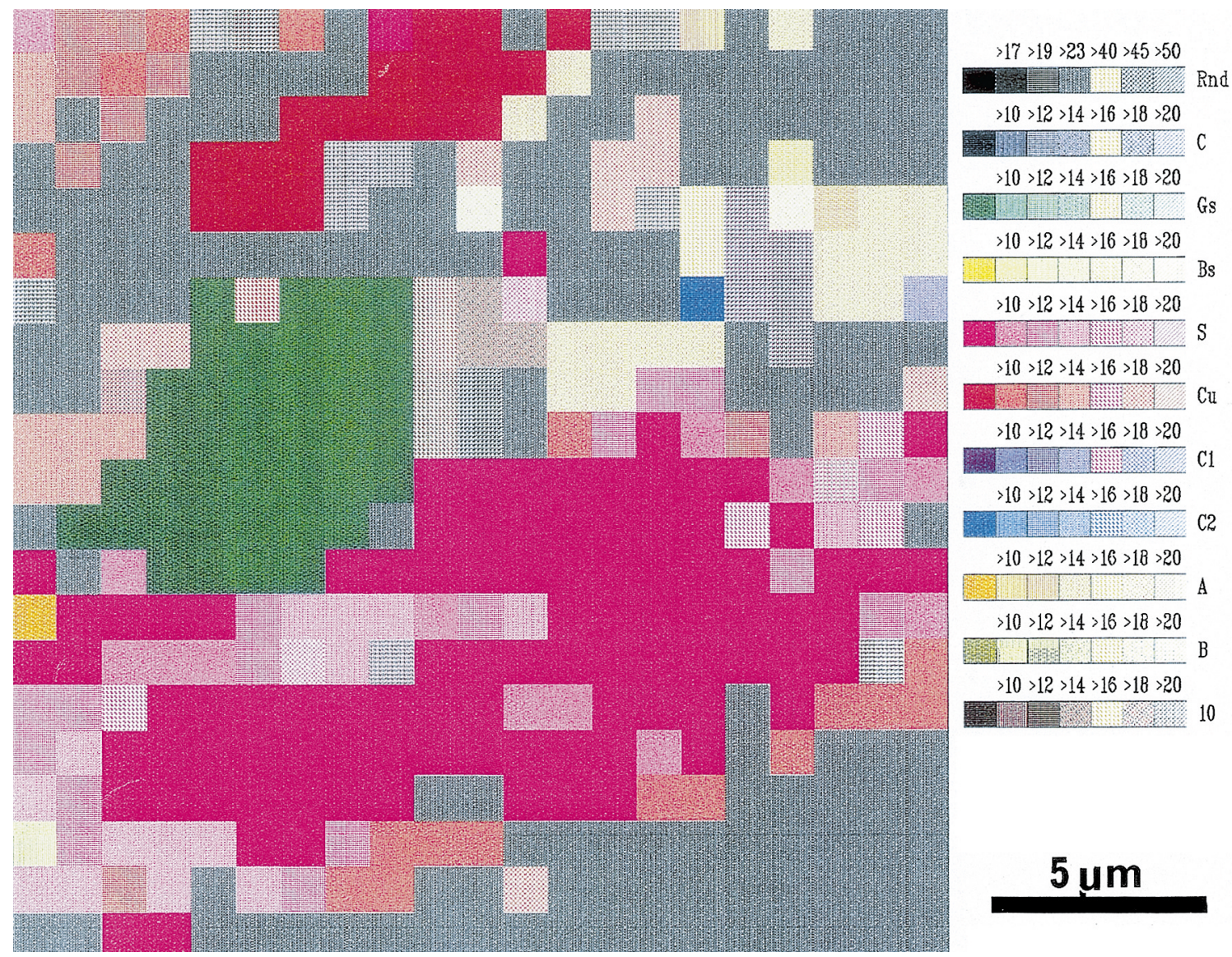

Fig. 9. Orientation image micrograph of aluminum (AA1050) $\mathrm{cr} 90^{\circ}$ and annealed for $600 \mathrm{~s}$ at $280^{\circ}$ in a bath furnace. Various orientations are represented by different colors. A nucleus is seen green color.

orientation relationships. This may be studied by the classic Beck experiment [70] where the size of artificially nucleated grains of all orientations are measured after growth in a well characterized deformed single crystal matrix. It is generally found that boundaries of specific types (e.g. tilt boundaries with a near $40^{\circ}\langle 111\rangle$ misorientation relationship) are highly mobile [70-73], whereas low angle boundaries are almost immobile $[2,14]$.

The driving force may also depend on the crystallographic orientation of the deformed matrix [26,74-77]. For example, in fcc metals of medium to high SFE, TEM observations typically reveal that the cell/subgrain size in regions with a $\langle 100\rangle$ orientation is larger than in other regions and X-ray diffraction line-broadening measurements suggest that $\langle 100\rangle$ regions are in a state of relatively less stress or strain than other regions [77]. One should, therefore, expect a lower driving force in these $\langle 100\rangle$ regions.

The above mentioned effects of orientation relationships on mobility and driving force are all fairly well known and well established. What consequences such orientation dependencies will have on growth of nuclei in heavily subdivided cold deformed matrices with many deformation induced high angle boundaries, however, have not been studied or considered in much detail before. The remaining parts of this section will, thus, be devoted to a short discussion of this question.

Already when a nucleus is formed in a cold deformed matrix, it will typically be surrounded by matrix material of different orientations at various segments along its boundary. An illustration of the 'segmentation' of nuclei/grain boundaries is given in Fig. 9. Here the EBSP technique was used to measure orientations in a 2-dimensional mesh along the RD (rolling direction) and ND in aluminum AA1050 cold rolled 90\% and annealed for $600 \mathrm{~s}$ at $280^{\circ} \mathrm{C}$ in a bath furnace. This annealing treatment corresponds to approximately $10 \%$ recrystallization. Selected orientations are represented by different colors. One nucleus is seen surrounded by deformed matrix characterized by a wide variety of orientations. This range of orientations is similar to the range shown in the deformed state, (Fig. 7). Similar results have been found by Haessner et al. [78].

During the growth of a nucleus/grain in a heavily subdivided deformed matrix, the boundary will constantly meet new types of deformation microstructures (volume elements with lamellar boundaries, DDW/ 
MBs, subgrains, cells, etc.) and new crystallographic orientations. Its growth conditions will, therefore, change constantly. Furthermore, even nearby segments of the boundary may experience quite different growth conditions because of small-scale variations in the deformed matrix. The nuclei/grains will, therefore, not have a constant mobility, $M$, or driving force, $\Delta f$, along their boundaries.

A consequence of the heavy subdivision of the deformed matrix into small volume elements of different orientations is that the misorientation relationship between a nucleus/grain and the surrounding deformed matrix cannot be characterized by a single set of misorientation parameters (e.g. one axis/angle pair). A distribution closer to that of a random distribution of boundary misorientations (i.e. a Mackenzie distribution [79]) should instead be expected. Experimental investigations confirm that misorientation distributions across nucleus-deformed matrix interfaces typically are very wide $[80,81]$. An example is shown in Fig. 10. Remarkable in many of these distributions is the relatively high fraction of low angle $\left(<15^{\circ}\right)$ boundaries. A low angle boundary develops whenever a nucleus/grain meets regions in the deformed material of almost its own orientation and as the mobility of the low angle boundaries is low, such regions will act as obstacles to the growth

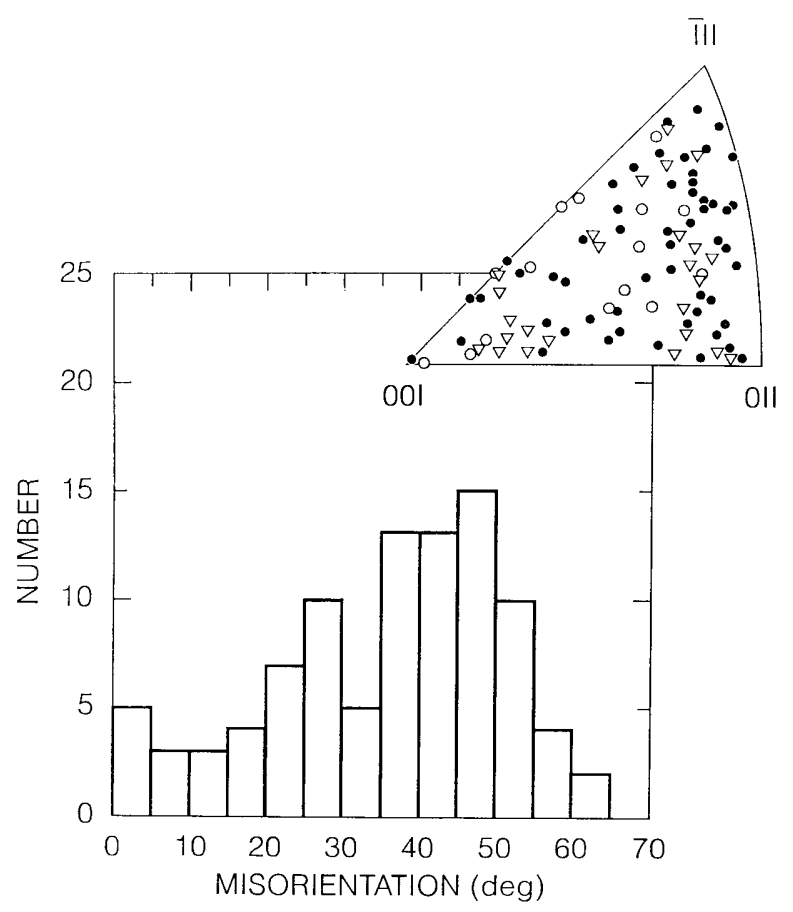

Fig. 10. Misorientations between growing nuclei/grains and the surrounding deformed matrix of OFHC copper $92 \% \mathrm{cr}$ and annealed at $121^{\circ} \mathrm{C}$ for $750 \mathrm{~s}$ (corresponding to $2 \%$ recrystallization). Misorientation angles are shown in histograms and the axes are showing unit sterographic triangles. In the sterographic triangles, different signatures are used for axes with different angles $(\theta)$ : $\theta \geq 35^{\circ}, \nabla$ $20^{\circ} \leq \theta<35^{\circ}, \bigcirc \theta<20^{\circ}$.
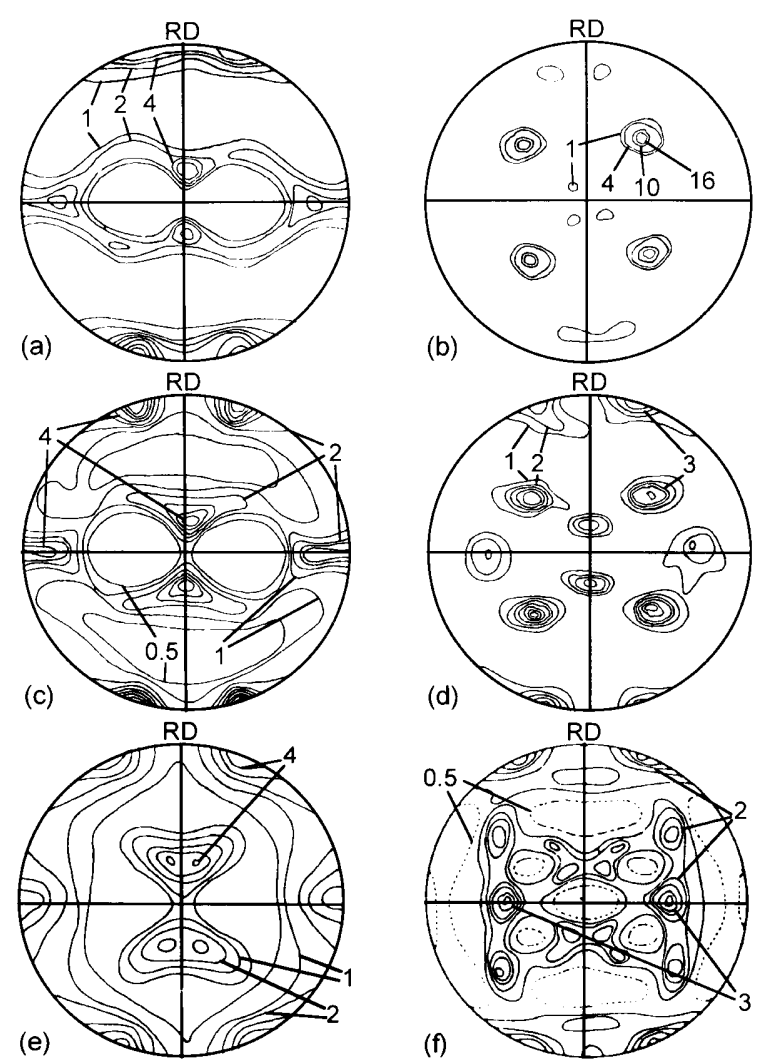

Fig. 11. $\{111\}$ pole figures for $\mathrm{Cu}$ and $\mathrm{Cu}-\mathrm{Zn}$ alloys before and after recrystallization. (a), (c), and (e) after rolling to $95 \%$ thickness reduction of (a) $\mathrm{Cu}$, (c) $\mathrm{Cu}-5 \% \mathrm{Zn}$ at room temperature, and (e) $\mathrm{Cu}-5 \% \mathrm{Zn}$ at $77^{\circ} \mathrm{K}$. (b), (d), and (f) show corresponding textures after recrystallization [83]. Note the strong cube texture for the pure copper, the appearance of a different recrystallization texture in the $5 \% \mathrm{Zn}$ alloy, and the retention of deformation texture components in the low temperature case.

of the nucleus/grain. This phenomena, referred to as orientation pinning, is discussed further in [34,82] (see Section 2.2.4) and has shown to be important for understanding differences in growth rates for nuclei/ grains of different orientations in typically observed cold deformed metals $[81,82]$.

\section{Issues in texture development and simulation of recrystallization}

\subsection{Texture evolution in recrystallization}

The changes in texture that occur during the recrystallization process can be dramatic in the sense that he previous texture of the deformed state is, in some cases, replaced by an entirely different texture. Fig. 11 illustrates the change from a strong fcc rolling texture in copper to a strong cube texture upon annealing [83]. Copper of high enough purity, rolled to a large reduction in thickness can exhibit an almost perfect cube 
texture after annealing, close to being a single crystal. Note that in the example given, the deformation texture lacks any component of the recrystallization texture. It also shows that as the deformation texture changes with alloy content, so the recrystallization texture also changes. In all three examples, however, the recrystallization texture is quite different from the deformation texture.

That a strong deformation texture is replaced with a different but strong recrystallization texture is consistent with our understanding of recrystallization as a process of nucleation and growth; new grains with (possibly) new orientations grow into the deformed structure, thereby eliminating the stored work of plastic deformation. The occurrence, or otherwise, of texture changes has been investigated and related to such parameters as alloy composition, strain level and deformation temperature [84]. The most notable feature of the experimental evidence is, however, the absence of sensitivity (of texture development) to the annealing conditions and the strong dependence on the prior plastic deformation. In other words, the entire recrystallization process is latent in the deformed state. It is this realization that has prompted the recent efforts to characterize the deformed state in sufficient detail that the nucleation of recrystallization can be related quantitatively to the deformed state.

As in a solid state phase transformation, growth of recrystallized grains can lead to drastic changes in microstructure and texture. One important difference from phase transformation, however, is that precise orientation relationships between deformed and recrystallized material are not observed. Approximate orientation relationships have nonetheless been used to make quantitative predictions of recrystallization textures, e.g. [85], with fair success. The basis for such an approach has been the observations of high mobilities of recrystallization fronts for certain misorientations, namely $40^{\circ}$ about $\langle 111\rangle$ in fcc metals [86], and $\sim 25^{\circ}$ about $\langle 110\rangle$ in bcc metals [87]. These observations have been made, however, on deformed single crystals which have been artificially nucleated to produce a wide range of misorientations at the recrystallization fronts. In practice, modelling the evolution of texture during recrystallization requires that only certain variants are selected from the available set. Although various hypotheses have been put forward [88], the validity of such approaches remains controversial because of the obvious microstructural heterogeneity of the process [80].

Recrystallization does not always lead to changes in texture, however, particularly when large volume fractions of second phase particles are present and pin boundaries, or when the deformation was axisymmetric. Some authors draw a distinction between continuous recrystallization (no long range motion of recrystallization fronts, therefore the deformed texture is preserved, to first order) and discontinuous recrystallization (long range motion of boundaries, with minority components often providing the source of nuclei). This point is discussed in more detail elsewhere in this review, Section 7. In the opinion of this author, however, it is more sensible to confine recrystallization to the case of motion of high angle boundaries (however defined, e.g. $\Delta \theta>15^{\circ}$ ). So-called continuous recrystallization then falls into the category of recovery processes, or extended recovery. Note that even if only subgrain coarsening occurs, there can still be measurable changes in texture if, for example, certain minority components are eliminated.

Studies of texture change during recrystallization have been concentrated in static recrystallization. Recently, however, work on hot torsion testing of interstitial-free steel [89] has shown that definite textures can develop during dynamic recrystallization. Fewer components are observed from dynamic recrystallization than in cold deformation and, at large strains, only the $\{112\}[111]$ component is observed. Despite the radically different conditions, texture development in dynamic recrystallization bears a strong resemblance to that of static recrystallization. Comparison with other materials, such as copper, $\mathrm{NiAl}$, and $\mathrm{Ni}_{3} \mathrm{Al}$ [90], shows that the texture evolution is highly dependent on the microstructural characteristics of the recrystallization process. Macroscopically, however, it is not possible to predict whether dynamic recrystallization will lead to a strong texture or the weakening of any prior texture.

Quantitative descriptions of texture evolution can be related to the boundary properties of specific components. By summing over the texture components in a recrystallizing system, it is possible, for example, to adapt the classical Kolmmogorov-Johnson-MehlAvrami (KJMA) description to texture evolution [91,92], where $F_{i}$ is the volume fraction recrystallized of the $i$ th component, and $\mathrm{d} F^{X}$ is extended volume fraction increment.

$\mathrm{d} F_{i}=\left(1-F_{\text {total }}\right) \mathrm{d} F_{i}^{X}$

$\mathrm{d} F=\sum_{i} \mathrm{~d} F_{i}$

The extended nucleation and growth then depends on the properties of the boundaries of the specific texture component which assumes that some average can be made over all the components present in the unrecrystallized region. Although this approach is useful in some cases, it is clear that more general approaches will be needed where the misorientation between unrecrystallized and recrystallized regions controls the boundary properties.

The generality of the importance of misorientation can be made apparent if one considers a simple form 
for the dependence of the actual volume fraction increment, $\mathrm{d} F$, on locally variable mobility, $M$ (a function of misorientation and boundary inclination), the associated increment of area, $\mathrm{d} S$, and driving pressure, $\Delta P$. The velocity of the recrystallization front must be integrated over the area of each recrystallizing grain and the contribution of all the grains summed for each orientation type.

$\mathrm{d} F_{1}=\mathrm{d} V_{i} / V_{0}$

Here the increments $\mathrm{d} F_{i}$ and $\mathrm{d} V_{i}$ are associated with a particular orientation type, $g_{i}$, and the system volume is $V_{0}$.

$d \mathrm{~V}_{i}=\mathrm{d} t \int_{S} v_{i} \mathrm{~d} S$

The velocity is written as $v_{i}$ and the volume increment for each orientation type has to be integrated over the grain boundary area associated with grains of that type.

$v=M \Delta P=M\left(g_{i}, g_{k}\right) \Delta P\left(g_{i}, g_{k}\right)$

The velocity depends on a mobility, itself a function of the misorientation and inclination of the boundary (i.e. recrystallization front), and the driving pressure for growth. Note that $\Delta P$ may be positive or negative depending upon whether one is considering growth of a recrystallized grain or (shrinkage of) an unrecrystallized grain. The change in each volume fraction is then given by the following.

$\mathrm{d} F_{i}=\frac{\mathrm{d} t}{V_{0}} \int_{S} M\left(g_{i}, g_{k}\right) \Delta P\left(g_{i}, g_{k}\right) \mathrm{d} S$

Note that the mobility is symmetric with respect to the difference in orientation across a boundary, but the driving pressure changes sign if the indices are reversed (i.e. $\mathrm{d} F$ may be positive or negative). For the simple case of a uniform stored energy, the driving pressure is only non-zero when a boundary between recrystallized and unrecrystallized material is considered. The driving pressure can, of course, also include capillarity and other effects, which may have a large effect at the nucleation stage.

As discussed elsewhere in this paper (see Fig. 9, for example), any recrystallized grain is expected to encounter a wide range of misorientations over its surface as it grows.

\subsection{Special textures in recrystallization}

Many examples exist of special textures arising from recrystallization and, although we now have qualitative explanations, much work remains to be done to devise quantitative, predictive description. Hutchinson's 1974 review laid out the basic features of recrystallization textures in fcc and bcc metals [74]. Most interest in steels has been focused on cold-rolled and annealed low carbon steels for deep drawing applications. Here, the technological requirement is generally to maximize the $\{111\}$ fiber component [93]. This can be accomplished but presents a challenge in terms of detailed scientific explanation that is an interesting contrast to fcc metals. The $\{111\}$ component has the highest Taylor factor of the standard texture components in rolling of bcc metals (and therefore higher stored energy). If nucleation occurs from regions of low stored energy growing into higher stored energy, by analogy to fcc metals, one might expect the $\{111\}$ component would be the most rapidly consumed during recrystallization by other components.

The development of $\langle 111\rangle$ fiber texture in bcc metals has been addressed by Hutchinson and Ryde [80]. Their measurements of misorientations at recrystallization fronts in low carbon steel clearly demonstrated that there is no special grain boundary character. Instead, the occurrence of nucleation in the $\langle 111\rangle$ component of the deformed structure must be the result of preferential nucleation, which appears to occur at prior grain boundaries [94]. This is significant, however, because it is important to understand why the deformed structure favors nucleation in the $\langle 111\rangle$ component.

In fcc metals, the cube component, which has the lowest possible Taylor factor, does successfully consume the higher Taylor factor rolling texture components (in the absence of shear bands and high densities of particles). The correlation of texture component Taylor factors with experimentally observed stored energy levels has been performed for both steel and copper, e.g. [95]. The challenges are then, first, to explain the preferential formation of $\{111\}$ textures from similar deformation textures in bcc and, secondly, to explain the occurrence of the cube $(\{100\}<011\rangle)$ texture in fcc metals [1].

The cube texture (in fcc metals) has received a great deal of attention in recent years thanks to its technological significance in earing control in aluminum alloys. The classical argument has focused on oriented nucleation (ON) versus oriented growth (OG) [69,74]. This distinction derived from early work on recrystallization of deformed single crystals which established that certain misorientations $\left(40^{\circ}\right.$ about $\langle 111\rangle$ in $\mathrm{fcc}, 30^{\circ}$ about $\langle 110\rangle$ in bcc) resulted in high mobility boundaries [86]. This observation did not, however, solve the problem of how to predict recrystallization textures. Other boundaries have been observed to migrate at relatively high rates under certain circumstances e.g. $45^{\circ}\langle 111\rangle$ and $24^{\circ}\langle 150\rangle$ in recrystallization of highly strained aluminium [358]. ON has enjoyed considerable experimental support from observations of cube-oriented volumes in deformed material and the similarity of final and partial recrystallization textures (considering only the recrystallized fraction) [96]. The observations of cube oriented regions in the as-rolled condition were 

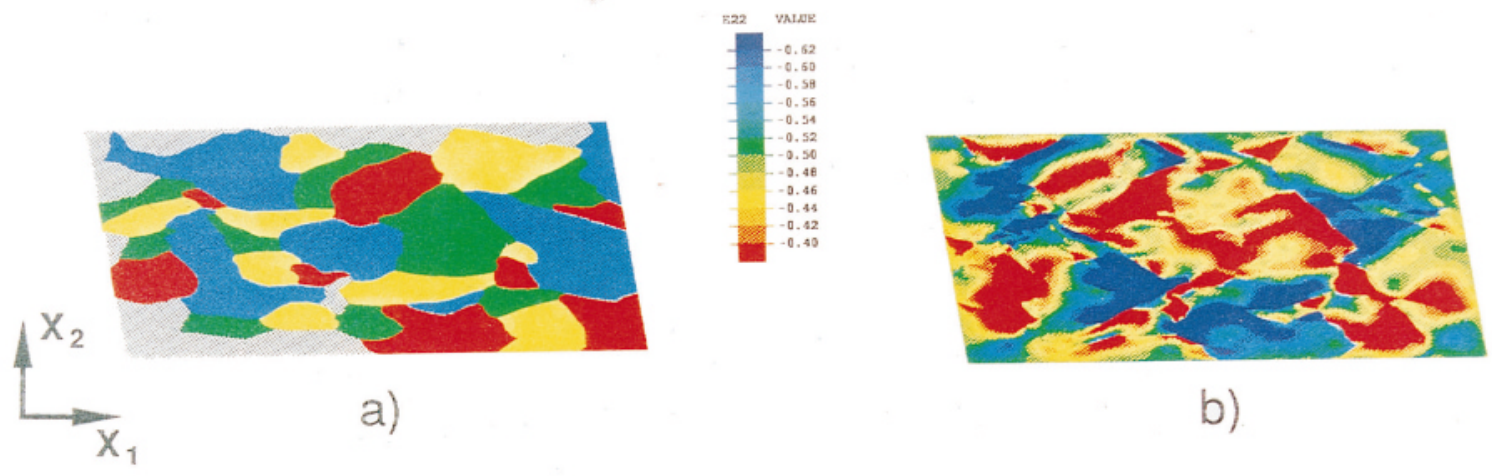

b)

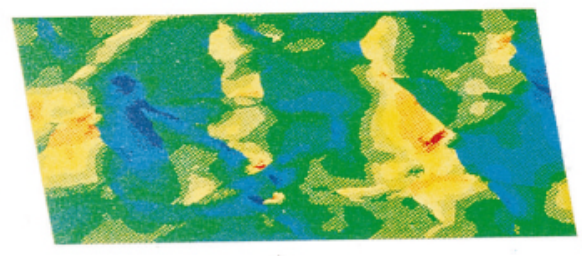

c)
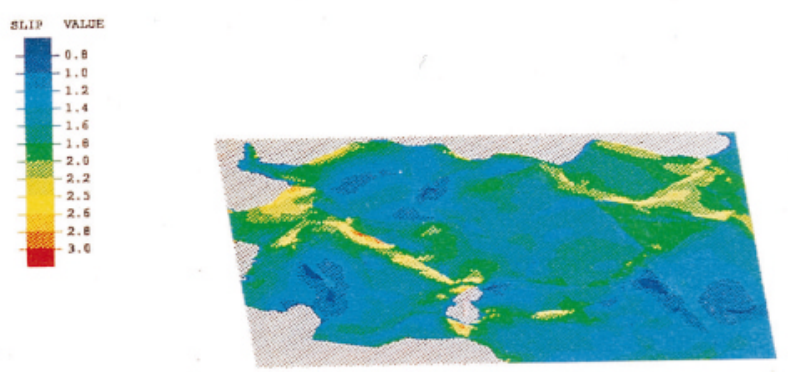

d)

Fig. 12. Results from simulation of plane-strain deformation of a polycrystalline block: (a) deformed grain shapes, (b) contours of thickness strain, (c) contours of shear strain, and (d) contours of accumulated slip. All three plots of the different measures of strain illustrate the heterogeneity of deformation and the tendency of localization of strain [39].

puzzling until it was realized that (a) many materials exhibit small but finite fractions of cube oriented material from the moment they are cast, and (b) the heterogeneity of deformation results in local stability of the cube orientation in transition regions [97].

The challenge remains to explain the details of cube orientation retention in rolling, which will be largely the province of careful solid mechanics coupled with polycrystal plasticity. For example, a recent finite element simulation of plane strain compression (i.e. rolling) of a multi-crystal body with several S-oriented grains and many elements per grain showed that transition bands can form which contain near-cube oriented material [40]. This modelling work needs to be coupled to a better experimental understanding of how plastic deformation leads to the generation of mobile, high angle boundaries that are required for nucleation to occur. Recent work by Panchanadeeswaran et al. [38] demonstrated that individual grains reorient during plastic deformation with appreciable scatter from the predictions of the Taylor model. Calculations with a finite element model using crystal plasticity confirmed the strong effect of neighboring grains on the behavior of each grain. Fig. 12 [39] illustrates the variation in local strain character based on a calculation of plane strain compression of a polycrystalline aluminum sample.
Resolution of the different behaviors of steel, as a representative bcc system, and aluminum or copper, as representative fcc metals, then depends on detailed investigation of the deformed state and its reaction to annealing. For fcc metals, the cube orientation has several special features that help to explain its predominance in recrystallization. One is its low Taylor factor which at least tends to minimize the total shear strain and therefore the stored dislocation density. In addition, it has been suggested [98] that recovery should be relatively quick because of the lack of elastic interactions between the (orthogonal) active slip systems in the cube orientation. Secondly, the cube orientation is symmetric with respect to the principal strain axes in rolling, and although it is metastable, might be expected to remain in the deformed structure as a transition between texture components that have formed by symmetric rotation (symmetric with respect to sample symmetry, that is). Lastly, FEM studies have shown that the heterogeneity of deformation at the scale of individual grains allows small cube-related volumes to remain in the deformed structure. In the bcc case, the $\langle 111\rangle$ component is a fiber texture which allows high angle boundaries between neighboring grains or subgrains within a single region of the $\langle 111\rangle$ component. 
This in itself does not explain the higher rate of nucleation in the $\langle 111\rangle$ component, so there is a clear need to perform both FEM calculations of the deformed state and to study the early stages of recrystallization (i.e. the formation of embryonic new grains) in different regions corresponding to the different deformation texture components. Another ill defined variable in the bcc case is the influence of second phase particles. Dispersions of small AlN particles, for example, present in many low carbon steels, may play a role in pinning certain boundaries. The recent work on aluminum [99] suggests that similar variations in grain boundary energy (and therefore pinning pressure from particles) should exist in other materials.

Further support for the importance of understanding the deformed state can be found in the recent work of Necker on rolled copper [100]. He observed a very strong dependence on prior rolling strain for the occurrence of the cube component, i.e. that strains over 2 are required to develop a strong cube texture (see Fig. 13). This strain dependence was recently confirmed in aluminum by Vatne et al. [101]. Although initial grain size has a marked effect, another key observation was that the growth rate of recrystallizing grains decreases with time. Despite the high purity $(99.995 \% \mathrm{Cu})$, the most likely explanation of the decreasing growth rates (also observed by many other workers) is that solute accumulates on the recrystallization fronts and slows them down. This suggests that high resolution electron microscopy (in terms of chemical analysis) on solute levels at recrystallization fronts is required.

Although microtextural analysis has become recently a very useful tool for investigating the recrystallized

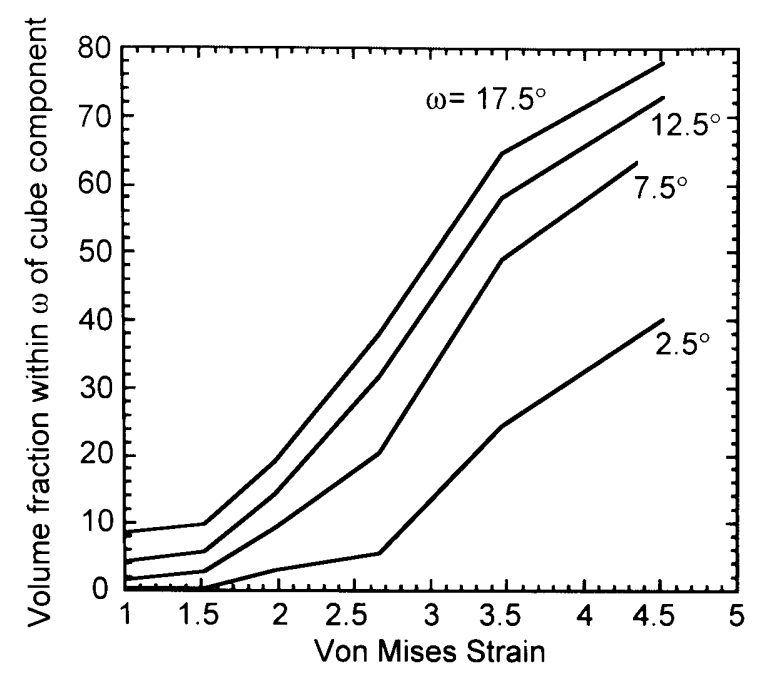

Fig. 13. Plot of volume fraction of the cube component in recrystallized copper as a function of the prior strain (in rolling) [100]. If the maximum angular deviation $(\omega)$ from exact cube is chosen to be greater than $7.5^{\circ}$, only small variations in cube volume fraction with strain are observed. state, it is still difficult to examine the deformed state in detail. Some recent results from Duggan and Vatne have suggested that the old work on growth selection in single crystals may hold the key to ON $[102,103]$. When the nearest neighbor relationships are investigated for cube oriented regions in deformed copper and aluminum, it appears that those cube regions that border on S-oriented material (i.e. have a $40^{\circ}\langle 111\rangle$ misorientation) are much more likely to lead to nucleation of new (cube oriented) grains. This has led these authors to support a 'micro-growth selection' theory. Note the (unexplored) link to the results of finite element modelling discussed above [40]. It is important to recall, however, that boundary energy and stored energy differences should be considered along with the boundary mobility [104]. This issue should be investigated further by careful electron microscopy, either with thick foil transmission microscopy or by high resolution orientation imaging microscopy in scanning electron microscopy.

Lastly, many issues in recrystallization reveal a fundamental gap in our knowledge of grain boundary properties. The nucleation process, for example, is critically dependent on the variation of mobility and energy with misorientation. Yet, the range of misorientations for which experimental data is available is limited to specific, symmetric misorientations such as $\langle 111\rangle$ or $\langle 100\rangle$ tilt boundaries [105]. The long established existence of special misorientation (with high mobility) has been loosely rationalized on the basis of the geometry of coincidence site lattices, despite the conflict between the particular angular dependence of mobility expected on the basis of the CSL approach and the experimental observations. Furthermore, the experimental evidence points to a dependence of properties on boundary plane in addition to misorientation [106]. Therefore, there is an obvious need to study grain boundaries, including low angle boundaries, over the complete fundamental zone [107]. These issues are discussed in more depth elsewhere in this review.

\subsection{Simulation of microstructural evolution during recrystallization}

The need for computer simulation of recrystallization is driven by two different needs. One is the need to be able to make quantitative predictions of the microstructure and properties of materials as affected by annealing. Such engineering-driven needs may only require a description of the average texture or grain size as its output. An equally important motivation for simulation, however, is the need for improved understanding of a phenomenon that is highly complex from a microstructural point of view. The changes in texture that occur upon annealing and their dependence on microstructural events serve to illustrate the importance of 
being able to model the recrystallization process in considerable detail. Indeed, if the microstructural evolution process inherent in recrystallization depends on the misorientation between like-oriented regions (in addition to the shape of their boundaries) then the importance of simulation techniques that can model both idealized and realistic microstructures is evident.

The application of computer simulation to grain growth and recrystallization was strongly stimulated in the early 1980 s by the realization that Monte Carlo models could be applied to problems of grain structure evolution. By extension of the Ising model for domain modelling of magnetic domains to the Potts model (with generalized spin numbers) it was then possible to represent discretely grains (domains) by regions of similarly oriented sets of material (lattice) points [108]. In parallel with this computer-based approach to modelling, there also occurred notable work on analytical models, especially by Abbruzzese and Lücke [109], which has been particularly useful for understanding the variation of texture (crystallographic preferred orientation) during grain growth processes. Bunge and Köhler [110] have used an analytical model based on variable growth rates of one texture component into another to simulate the evolution of texture during primary recrystallization. The critical issue addressed by Humphreys' [111] extension of grain growth theory is that of coarsening of subgrain networks and under what circumstances one expects to observe nucleation of recrystallization. This view of nucleation as simply non-uniform coarsening (i.e. abnormal subgrain growth) is significant for its blurring of the distinction between continuous and discontinuous recrystallization.

There are four current methods of mesoscopic simulation for recrystallization. The first, geometrical, method addresses primarily the final microstructural state; it can be used to investigate microstructural evolution, provided that one is not concerned with the effect of grain growth occurring in parallel. Such models of recrystallization were first elaborated by Mahin and Hanson [112] and then developed further by Frost and Thompson [113]. Furu [114] and Juul Jensen [115] have recently extended these models to predict grain size and texture development during recrystallization.

The second method, based on network models, has not been developed extensively but shows promise as an efficient way to represent microstructural evolution in discretized form [116]. Such models have been developed to a high degree by Fradkov and others [117]. These models have the strength that they abstract a key feature of the grain structure, i.e. the vertices, and are therefore efficient because only the vertex motion needs to be calculated. They have some limitation when second phases must be considered, however (see also the work of Frost [118]). More recently Humphreys [116] has applied the network model to the nucleation pro- cess in recrystallization by considering coarsening processes in subgrain networks. The properties of the boundaries in the network are assumed to vary with misorientation such that the boundary energy varies according to the Read-Shockley equation. Strain induced boundary migration was simulated, for example, by placing a high angle boundary in a network of low angle boundaries. Fig. 14 shows a series of microstructures for simulations of abnormal grain growth in which both the energy and the mobility of the boundary of the central grain vary from the (otherwise uniform) properties of the boundaries in matrix [119]. A small change in boundary energy leads to a significant difference in behavior; in the case where the boundary energy is low, abnormal grain growth occurs but in the higher boundary energy case, abnormal grain growth does not occur and the central grain is absorbed into the general structure.

The third method, cellular automata, also discretizes the microstructure and has also been successfully applied to recrystallization, e.g. by Hesselbarth [120]. Physically based rules are used to determine the propagation of a transformation (e.g. recrystallization, solidification) from one cell to its neighbor. It has been used to verify the effect on non-uniform stored energy on lowering the apparent exponent in a KJMA analysis. It has not, however, been used in as wide a range of problems in recrystallization as has the fourth, Monte Carlo method [121-123]. This latter approach relies on the Potts model to both discretize the structure and simulate boundary motion via an energy minimization procedure. The effect of varying nucleation and growth conditions, heterogeneous stored energy, particle pinning effects and dynamic recrystallization have all been investigated with the Potts model.

\subsection{Future direction for simulation of recrystallization}

New approaches to the simulation of microstructural evolution are becoming available. We review some of them here to illustrate where the practice of recrystallization modelling may progress in the future.

Although 3D simulations might reasonably be expected to confirm the classical kinetics, there is an evident need for extending the existing $2 \mathrm{D}$ work in order to investigate the effect of non-uniform properties and structure. For example, the kinetics of abnormal growth may well be different in $3 \mathrm{D}$, which is important to the understanding of nucleation of new grains. Also, the interaction of grain boundaries with particles depends on dimensionality; in two dimensions, particles can remove curvature more efficiently than in three dimensions [124]. Recent simulation work [125] shows that low particle densities can lead to microstructural states in which an abnormally large grain can grow into a matrix of fine grains that is pinned; this happens 

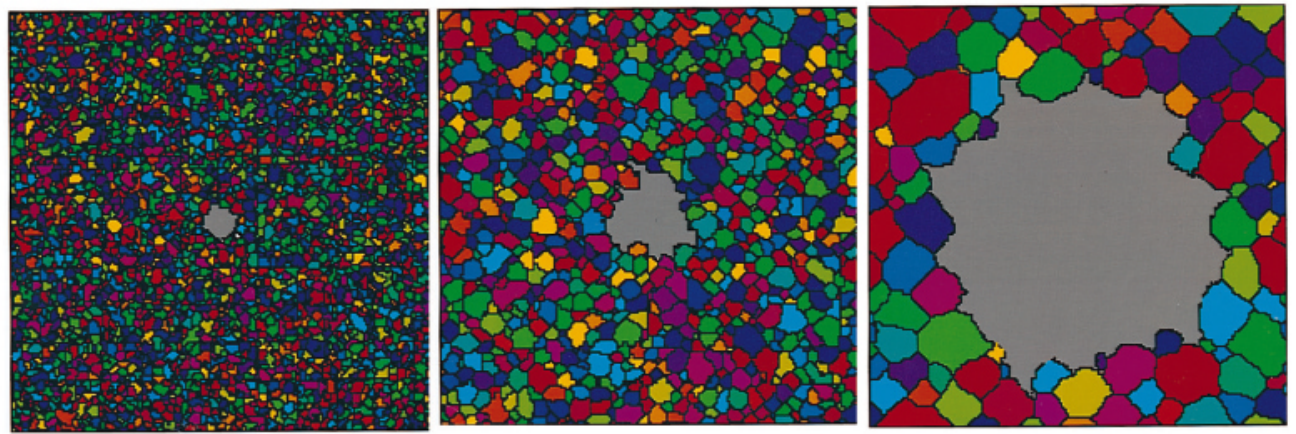

(a) $400 \mathrm{MCS}$

7,000

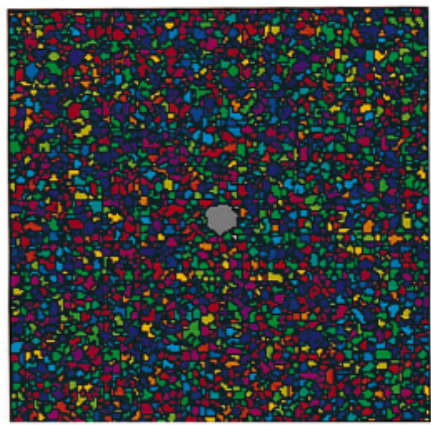

(b)

\section{MCS}

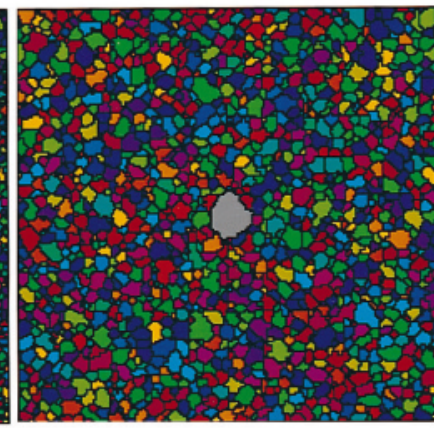

4,000
70,000

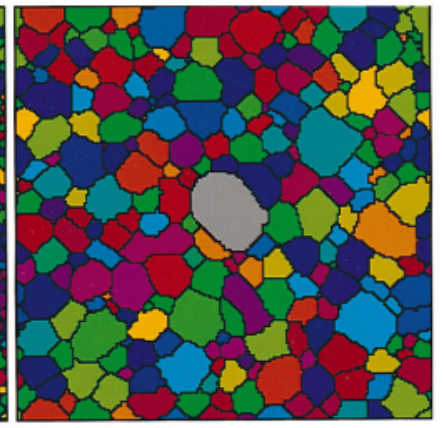

50,000

Fig. 14. Results of calculations with the Potts model [86]: (a) evolution of an abnormal grain with boundary mobility twice that of the matrix boundaries $(\mu=2)$ and energy $40 \%$ lower $(\Gamma=0.6)$; (b) evolution of an abnormal grain with boundary mobility twice that of the matrix boundaries $(\mu=2)$ and energy $30 \%$ higher $(\Gamma=1.3)$.

because, in three dimensions, particle inhibition is effected by pinning of the smallest grains only. This suggests that the nucleation of new grains in the presence of second phase particles is microstructurally complex; see Section 5 for a more complete discussion.

Also, it is evident that the growth of recrystallized grains into a deformed structure leads to a wide variety of misorientations. Orientation pinning, or variant inhibition has been postulated to constrain growth because of the creation of low angle, low mobility interface when a recrystallizing grain encounters similarly oriented material [34]. However, the range of misorientations encountered during the growth to impingement of each recrystallizing grain is considerable as discussed above. There is also experimental evidence in copper [99] for a dependence of boundary energy on misorientation based on the observation that only certain grains are able to grow into a deformed, particle containing single crystal. The evaluation of misorientation was, however, based on average misorientation between the new grain and the deformed matrix and a significant spread was observed about the preferred $\langle 111\rangle$ misorientation axis. Three-dimensional modelling is clearly needed in order to address these complexities and to investigate percolation effects in the growth of new grains.
Alternative potential methods of simulating recrystallization exist. The mathematics community has developed numerical approaches to finding minimal surfaces and related problems. Surface Evolver, for example, is available as a public domain program through The Geometry Center at the University of Minnesota. This program treats surfaces as tessellations of triangles and can either find minimum energy configurations or evolve sets of surfaces towards minimum energy states.

L.-Q. Chen has described a new method for simulating microstructural evolution that is based on the diffuse interface approach pioneered by Allen and Cahn [126]. This method has been demonstrated to reproduce the essential features of normal grain growth for isotropic boundary properties, that is to say compact grain shapes, and square root of time coarsening kinetics. This method shows same promise for modelling recrystallization in complex systems, especially those exhibiting ordering. Other types of simulation are relevant to recrystallization because of the dominant effect of the deformed state. One need is to be able to predict microstructure at the dislocation network level, and how such networks evolve during annealing. Canova et al. [364] are able to model large sets of dislocations in 
a deforming crystal where the dislocations are modeled as line defects with the appropriate line tension and interaction forces. These models are currently being used to investigate the development of dislocation structure arising from plastic deformation. They may also be suitable for investigating the relative rates of recovery in different orientations. For example, many authors have postulated a more rapid recovery rate in cube oriented grains in f.c.c. metals but no quantitative predictions for this exist.

At a more macroscopic level, it has long been known that plastic deformation, even in a single-phase metal, is heterogeneous. Although it is easy to see that variations in Taylor factor might cause some grains to deform more easily than others, it is only recently that increased computing power has allowed finite element (FEM) simulations to be applied to predictions of the heterogeneity of plastic deformation, as illustrated in Fig. 12. It is also now feasible to incorporate single crystal properties into the FEM models, which allows deformation to be simulated at the grain scale, as discussed above. The overarching aim of such studies should be to determine the statistics of how microstructural elements develop that are known to be highly likely to generate new grains during recrystallization.

Progress has been made towards coupling the results of FEM deformation modelling and recrystallization modelling. Sarma et al. [127] have demonstrated that it is possible to take the results of three-dimensional FEM calculations with constitutive relations based on crystal plasticity and use those results to define both stored energy and misorientation distribution for a Monte Carlo simulation of recrystallization. Such calculations illustrate the advances that are being made in linking different length scales and simulation techniques together.

A subsidiary point is that we know very little about the development of large misorientations during plastic deformation. The magnitude and distribution of misorientation is critical to nucleation of new grains, see Section 2. Hughes [44] finds that the average misorientation present increases as the square root of the strain in Al. It would be useful to have a physical basis for the result and the finite element models may offer us some clues, as well as addressing the issue of transition band development, discussed in Section 3.

In summary, the strong motivation for macrostructural simulation is to be able to model recrystallization as a microstructurally complex process. Any new grain encounters a wide range of orientation as it grows: therefore, the character of the interface between recrystallized and unrecrystallized material changes continually as the recrystallizing grain encounters new orientations in the deformed material. Other microstructural features such as a second phase particles and solutes influence the motion of recrystallization fronts and also should be incorporated into simulations. We should expect to have to analyze the results on a statistical basis in order to abstract new understanding of the recrystallization process.

\section{Second-phase particles and recrystallization}

\subsection{Introduction}

There are three reasons why an understanding of the effects of second-phase particles on recrystallization is important: (a) most industrial alloys contain secondphase particles; (b) such particles have a strong influence on the recrystallization kinetics, microstructure, and texture; and (c) by a combination of alloying and materials processing, it is possible to control the distribution of second-phase particles in the microstructure relatively accurately. If the effects of these particles on the annealing behavior are understood, then the metallurgist is able to use second-particles as a method of controlling the grain size and texture during thermomechanical processing.

The effects of particles on recrystallization are complex. During deformation, particles will affect the deformation microstructure and texture through effects such as an increase in dislocation density, the production of large deformation heterogeneities at larger particles, and the alteration of the homogeneity of slip, e.g. shear bands. During annealing, the primary effect of closely spaced particles is to pin grain boundaries (Zener pinning), but the deformation heterogeneities at large particles may be sites at which recrystallization originates (particle stimulated nucleation or PSN).

Apart from Zener pinning, the mechanisms of recrystallization in two-phase alloy do not differ from those in single-phase alloy and our understanding of the underlying physics is limited by the same problems, the key areas being of lack of quantitative and predictive information about the nature of the deformed state and an almost total ignorance of the properties of moving low or high angle grain boundaries. As is the case for single-phase alloys, there has been a rush to produce so-called 'physically based' theories or models for the various annealing phenomena, but in the absence of a comparable effort aimed at determining the underlying physical processes which occur during deformation and annealing, such models are still largely empirical and, therefore, of limited value. Nevertheless, we have a reasonable understanding of the effect of second-phase particles on recrystallization and how to control the resulting microstructure and texture by the use of particles $[2,128,129]$. 


\subsection{Discussion}

\subsubsection{Large $(>1 \mu m)$ particles}

5.2.1.1. Deformation. During the deformation of a particle-containing alloy, the enforced strain gradient in the vicinity of a non-deforming particle creates a region of high dislocation density and large orientation gradient (particle deformation zone or PDZ), which is an ideal site for the development of a recrystallization nucleus. Experimental measurement of the particle deformation zones is very difficult and there have been very few detailed or systematic measurements of the size, shape, or orientation of these zones [2]. However, it is known that they extend to a distance of about a diameter from the surface of the particles and that they may be misoriented by tens of degrees from the adjacent matrix. Recent work on the deformation of particle-containing single crystals $[130,131]$ has shown that, in addition to the lattice misorientations very close to the particles small deformation bands (particle deformation bands or PBDs) may be formed at the particles and these can extend for more than ten particle diameters. These bands are regions which are destabilized by the presence of the particle, and which rotate during deformation towards stable orientations. A schematic diagram of the deformation structures at a large particle is shown in Fig. 15.

Simple models for the formation of particle deformation zones which attempt to account for the development of orientation gradients at the particles by considering the effect of a non-deformable particle on the local slip activity have been proposed, see [2]. However, these models cannot predict the shape of the particle deformation zones.

Further progress in modelling the formation of particle deformation zones is being made with the use of fine scale-finite element modelling and Fig. 16 shows the

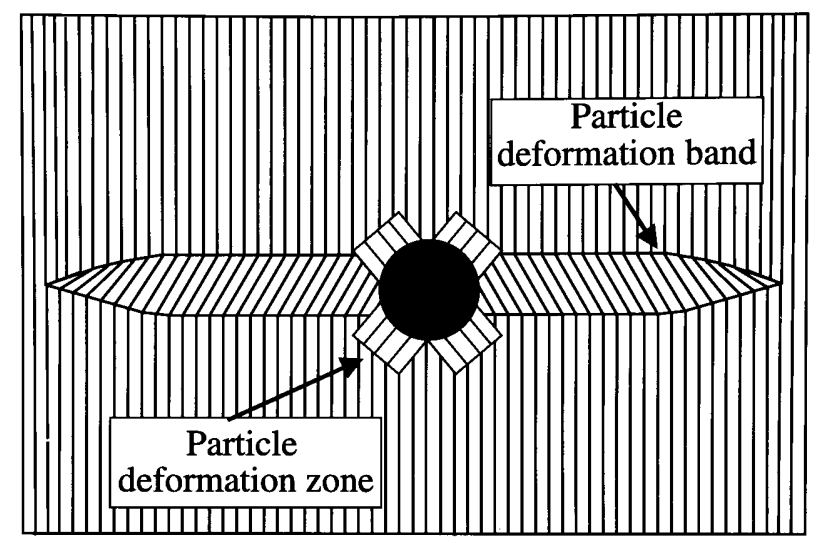

Fig. 15. Schematic diagram of the deformation structures at large second-phase particles.

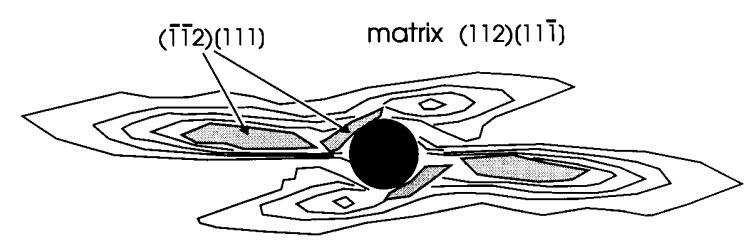

Fig. 16. The orientations close to a spherical particle in an $\{001\}\langle 110\rangle$ crystal, as calculated by FEM (courtesy of P. Bate).

predicted lattice rotations at spherical particle in a crystal of orientation $\{001\}\langle 110\rangle$. This type of modelling, which will also predict the rotations of the particle itself, is sensitive to the work hardening behavior assumed for the matrix, to the shape and interface of the particle, and to the boundary conditions of the model.

It must be emphasized that until the details of the particle deformation zones and their dependence on particle size, strain, and orientation are known, recrystallization models, which inevitably require the deformation structure as a starting point, cannot be fully quantitative and further experimental and theoretical work in this area is required.

\subsubsection{Annealing-particle stimulated nucleation} $(P S N)$. The condition under which PSN can occur are reasonably well documented $[2,129,131]$ and the mechanism of recrystallization is though to be similar to that at an orientation gradient in a single-phase alloy. During annealing after low temperature deformation, it is thought that the step is the growth of the new grain away from the environment of the particle. However, during deformation at high temperatures, the deformation zones required to originate recrystallization may not be formed and the process may be nucleation limited. The analysis shown in Fig. 17 is found to be in reasonable agreement with experiments on several aluminum alloys.

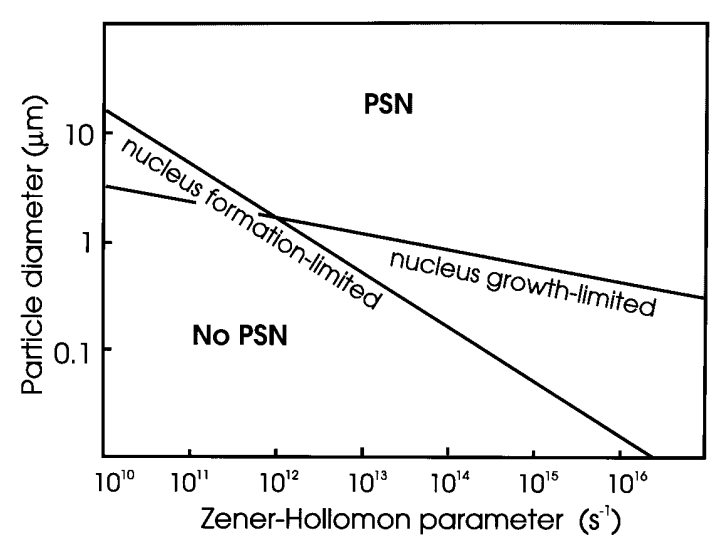

Fig. 17. The effect of deformation conditions on PSN [2]. 

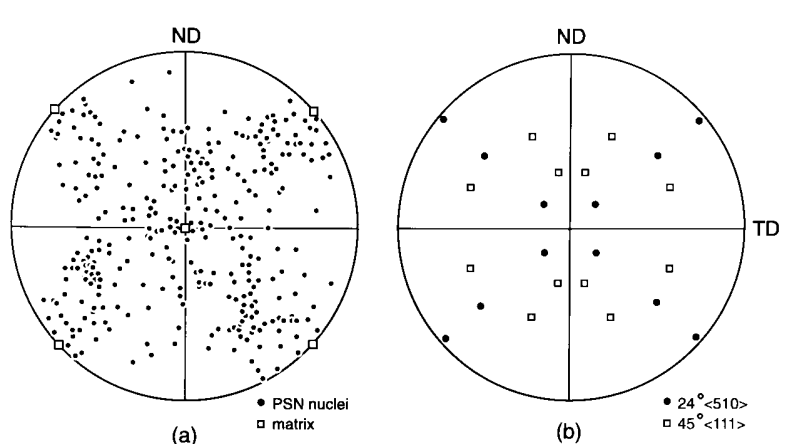

Fig. 18. 200 polefigures of recrystallized grains in an $\mathrm{Al}-0.8 \% \mathrm{Si}$ crystal of orientation $\{011\}\langle 100\rangle$ deformed to $\varepsilon=1.4$ and annealed at $300^{\circ} \mathrm{C}$ for $90 \mathrm{~s}$. (a) PSN nuclei and matrix orientation. (b) Average orientations of the fast growing grains which dominate the final microstructure [131].

There is a need for further basic experimental work on the conditions and kinetics of PSN and, in particular, the effects of particle size and shape on PSN need to be clarified.

The orientations of PSN grains is important as they contribute to the recrystallization texture. Work on the single crystals shows that the PSN orientations are broadly spread from the initial crystal orientation, as shown in Fig. 18. In highly deformed polycrystal most work shows the nuclei to be of almost random orientation. This has important practical consequences as it shows that by controlling the occurrence of PSN, it is possible to control the amount of random texture in the material. Most other recrystallization mechanisms produce particular texture components, and PSN is one of the few mechanisms of randomizing texture.

The contribution of PSN to the final recrystallized microstructure and texture is not entirely clear. In particulate aluminum composites containing large (3-5 $\mu \mathrm{m})$ particles, the grain size is small and the texture random, as would be expected. However, in conventional alloys it is usually found that the fraction of large particles at which PSN successfully occurs (PSN efficiency) is often low and typically $\sim 1 \%$ and it is also found that the final texture is sharper than would be expected from the PSN orientation $[2,131]$. The reasons for this are still a matter of discussion and the various possible reasons include:

- Preferential growth in the early stage, of other types of nuclei such as cube grains due to their local environment.

- Selection of certain orientations from among those produced by PSN.

- Preferential nucleation at particles in favored sites such as grain boundaries.

Although it has clearly been demonstrated that growth selection from among the PSN orientations occurs during the annealing of single crystals, its role in polycrystal is unclear, and further research is required in this area.

\subsubsection{Small or closely spaced particles}

It has long been established that recrystallization is prevented or delayed by a dispersion of closely spaced second-phase particles, due to the pinning (Zener drag) of both high and low angle boundaries, see e.g. [2]. The influence of the particles is expected to depend on a number of factors and, in particular, on whether the particles are present during deformation or whether they form during the subsequent anneal before recrystallization occurs.

\subsubsection{Annealing of a supersaturated solid solution.} Pioneering work on the annealing of supersaturated solid solutions was carried out by Hornbogen and colleagues [132] who showed that the interaction between precipitation and recrystallization was complex. At high annealing temperatures, recrystallization is likely to be complete before precipitation occurs and the particles, therefore, do not influence the recrystallized microstructure and texture. At lower temperatures, precipitation generally occurs before recrystallization and precipitates form on the deformed or recovered substructure, thereby inhibiting both recovery and recrystallization. A recent investigation of supersaturated $\mathrm{Al}-1.8 \% \mathrm{Cu}$ [133] showed that the recrystallization texture was strongly dependent on the strain. After lower strains $(<90 \%)$, there was significant retained rolling and cube textures. However, after larger strains, the dominance of shear banding during deformation resulted in $\mathrm{P}, \mathrm{Q}$, and Goss recrystallization texture components.

There has also been a considerable amount of research by the Trondheim group on the annealing of supersaturated commercial aluminum alloys, e.g. $[134,135]$. In such alloys, there are large intermetallic particles of the type associated with PSN in addition to the particles precipitating during the recrystallization anneal. The results show that a much stronger texture is achieved during low temperature annealing when precipitation is occurring than when it is not. In $\mathrm{Al}-\mathrm{Mn}$ AA3103 [135] precipitation is associated with a strong cube component and a large elongated grain structure; in $\mathrm{Al}-\mathrm{Mn}-\mathrm{Mg}$ [134] precipitation was associated with cube, $\mathrm{P}$, and ND-rotated cube texture components. In both cases the recrystallized components had a $40^{\circ}$ $\langle 111\rangle$ relation to the deformed matrix. Measurements of grain sizes in partly recrystallized samples confirmed that the growth rate of PSN grains was slower than that of the $40^{\circ}\langle 111\rangle$ components and a large spread of grain sizes with island grains was observed. As in both cases the precipitation was inferred from resistivity changes, no direct correlation with the microstructure could be made, although it was suggested that the 
growth differences were due to preferential precipitation on the boundaries of the growing PSN grains. For both alloys, high temperature annealing, during which no precipitation was expected, resulted in a fine grain size and a more random texture.

\subsubsection{Annealing of alloys containing stable and closely} spaced particles. Alloys contains stable dispersions of closely spaced particles and in which Zener pinning retards recrystallization are generally characterized by a large grain size see [2]. The recrystallization behavior depends on whether the particles are deformed during deformation. If so, then the extensive shear band formation dominates the recrystallization process and Goss, $\mathrm{P}, \mathrm{Q}$, and, to a lesser extent, cube components are formed [136]. It is sometimes found that a strong rolling component is retained in the recrystallization textures of alloys containing small non-deformable particles which were present before deformation [137].

5.2.2.3. Bimodal particle distributions. It is difficult to interpret the behavior of both types of material discussed above because in the supersaturated solid solutions the amount of second-phase varies with time and is inhomogeneously distributed. In the case of stable small particles, the origin of the recrystallized grains is unknown. A simpler case is expected to be a bimodal alloy, which contains both very large particles at which PSN occurs and also a distribution of small non-deformable particles which pin the boundaries. In this case the only change in phase distribution during isothermal annealing is the gradual coarsening of the small particles.

Al-Si alloys provide a good model for such a microstructure and Chan and Humphreys [138] showed that, despite the presence of the very large $(>5 \mu \mathrm{m})$ particles at which PSN occurred, the recrystallization kinetics were determined by the pinning effects of the small particles. The final grain size was very large and many of the large particles were associated with island PSN grains. Recent work on similar alloys [139] has confirmed the earlier work and extended them by texture and microtexture investigations. The bimodal alloys recrystallized to a large grain size produced 'island' PSN grains and had relatively strong cube textures. Similar alloys containing only the large particles showed a weaker cube component and had much smaller grain size and alloys containing only the small particles had very strong cube textures and a large grain size.

The strong correlation between texture and grain size is very similar to the supersaturated alloys discussed above. Comparison may also be made with powered metallurgy produced aluminum, which contains a fine dispersion of $\mathrm{Al}_{2} \mathrm{O}_{3}$ particles and in which very large grains and a strong cube texture are formed on recrystallization [140].
It is, therefore, clear that in a large number of alloy systems, a fine particle dispersion which retards recrystallization also produces a stronger cube texture and a larger grain size. The larger grain size, island grains, and texture components which are related by $\sim 40^{\circ}$ $\langle 111\rangle$ to the deformed texture, strongly indicate some selection of favored grains. Such growth selection would be favored by the very large initial grain size and the homogenizing effect of the fine dispersion, both of which would result in large volumes of coherent orientation in the deformed microstructure.

In the alloys discussed above, the explanation for the advantage of cube over PSN grains cannot be due to preferential precipitation, such as was proposed for the supersaturated alloys. A hitherto unconsidered factor which may be important is the possibility of an orientation dependence of the Zener pinning pressure $\left(P_{\mathrm{Z}}\right)$. It has been shown in a copper alloy [99] that $P_{\mathrm{Z}}$ is orientation dependent, with boundaries of $\sim 40^{\circ}\langle 111\rangle$ having a $5-10 \%$ lower pinning pressure than other boundaries. This is not a boundary mobility difference and is thought to arise from a difference in boundary energies. Although this is only a small effect, it may play a critical role when the recrystallization is controlled by Zener pinning, when the total driving pressure $\left(P_{\mathrm{D}}-P_{\mathrm{Z}}\right)$ is small, and any fluctuations in $P_{\mathrm{Z}}$ give a growing grain a large advantage in the early stages of recrystallization. Models incorporating this factor have confirmed that, in such alloys, a very small orientation dependence of $P_{Z}$ may have a large influence on the microstructure and texture.

\section{Conventional dynamic recrystallization (DRX)}

\subsection{Introduction}

In the current context, dynamic recrystallization (DRX) refers to the occurrence of recrystallization during deformation. When this type of 'annealing' process is taking place, both nucleation as well as growth (grain boundary migration) take place while the strain is being applied. It has been known since the late 50 s or early 60s [141] that the flow curves associated with DRX can by cyclic or 'single peak'. It was shown subsequently that cyclic deformation indicates that grain coarsening is taking place (Coarsening ends when the cycles are 'damped out'), while single peak flow is associated with grain refinement [142].

The physical mechanisms responsible for DRX are similar in many respects to those controlling static recrystallization (SRX). Indeed, the computer modelling of DRX often involves sequential deformation steps followed by increments of SRX in the absence of straining. Accordingly, many of the topics discussed below are also of importance with respect to improving 
our understanding of SRX. The following topics will now be considered in turn: oriented nucleation, selective growth, the role of twinning, texture development during DRX, postdynamic static and metadynamic recrystallization, the interaction between precipitation and DRX, the effect of solute elements on DRX, and the kinetics of DRX and SRX after high temperature deformation.

\subsection{Oriented nucleation}

The mechanism involved in the nucleation of DRX has not been positively identified. With the availability of EBSD techniques, it is expected that rapid progress will be made in this area. A question that always arises is 'low stored energy' versus 'high stored energy' nucleation. The available data for both fcc [143] and bcc [144] metals suggest that low stored energy nucleation is involved. This can be because, at the high temperatures required for DRX, the high stored energies commonly developed during cold working cannot readily be generated. As a result, the subgrain coalescence mechanism associated with high stored energy nucleation is not likely to play a role and SIBM (strain-induced boundary migration) or some other low energy mechanism is probably of primary interest instead.

A nucleation mechanism that is observed to operate in cold rolled and annealed aluminum alloys is PSN (particle stimulated nucleation). This is discussed in more detail in the previous section. It is of interest here because, although aluminum alloys do not normally undergo DRX (because of their low hot worked dislocation densities), the nucleation and growth of new grains can indeed be induced in the presence of sufficient quantities of hard second phase [145]. These appear to raise the local dislocation densities and lattice curvatures above the critical levels needed for the initiation and propagation of DRX. Similar nucleation events attributable to PSN are likely to take place in the other fcc alloy systems, which are capable of undergoing DRX; this is clearly a fertile topic for further investigation.

We turn finally to the influence of shear bands, deformation bands, intersecting transition bands, etc. on the nucleation process. Here again little is known, in part because austenite, which is the most important fcc material from the point of view of DRX, is unstable at room temperature. It can be surmised that intense flow localizations of the type referred to above are less common at elevated than at ambient temperatures. This is partly because the associated lower dislocation densities are less unstable and hence provide a lower driving force for sudden softening events. In a similar manner, the higher rate sensitivities applicable to high temperature deformation can also be expected to retard flow localization. Nevertheless, there is clear metallographic evidence for the presence of shear bands in hot rolled austenite [146]. The appearance of shear bands in ferrite warm rolled at $700^{\circ} \mathrm{C}$ is illustrated in Fig. 19 [147].

The characteristics of the nucleation of DRX at such locations (if it occurs) is of industrial as well as scientific interest. These could be determined on model materials such as copper or nickel, which do not undergo a phase change on cooling. Such information would help to clarify the role of shear band nucleation on texture development, a topic that is taken up in greater detail below.

\subsection{Selective growth}

The debates about oriented nucleation versus selective growth continue unabated in the cold working and annealing literature! With regard to DRX, the results obtained to date indicate that selective growth plays a significant role in fcc metals [143], but not bcc metals [144]. Whether or not a particular boundary class (or misorientation relationship) is associated with a mobility edge thus also appears to depend on crystal structure, as well as on the dislocation densities and internal stresses generated in the grains (high in fcc, low in bcc). This is a topic area where orientation imaging microscopy (OIM) can be very useful and where there is a need for both data and careful analysis.

There are two related types of boundaries that can participate in selective growth: (i) PM (plane matching) boundaries were first described in detail by Watanabe [148] and are illustrated in Fig. 20(a); and (ii) coincident site lattice (CSL) boundaries are much better known than PM boundaries and can also participate in selective growth.

There is recent evidence $[143,149,150]$ that selective growth, when it occurs, involves 'variant selection'; that is, that not all geometrically equivalent boundaries participate in the recrystallization process. When selective growth by either the PM or the CSL mechanism is taking place, the replacement of a deformed grain by a

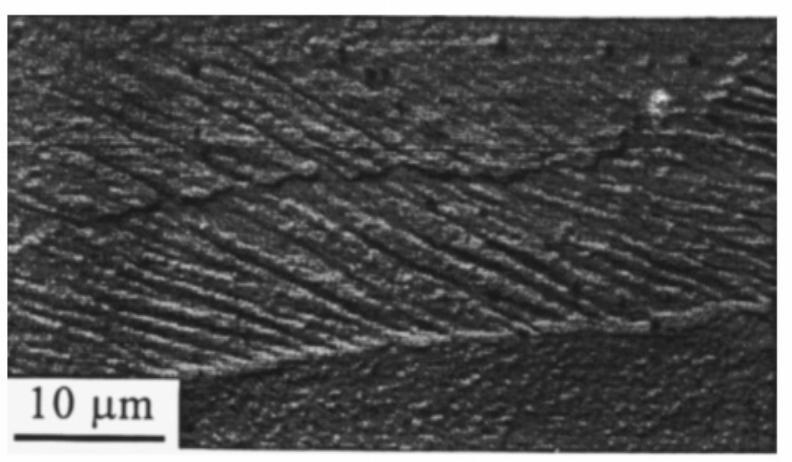

Fig. 19. Example of in-grain shear bands in a Ti-stabilized interstitialfree steel warm rolled at $700^{\circ} \mathrm{C}$ in one pass to a reduction of $65 \%$ [147]. 


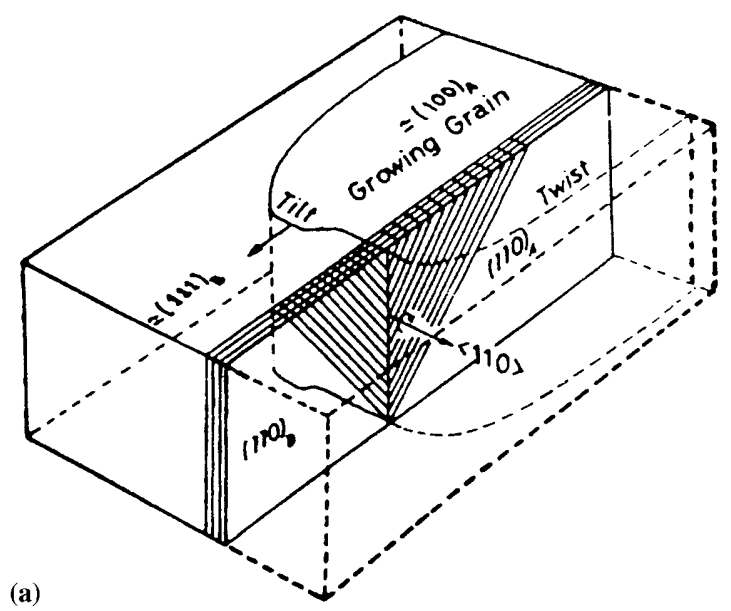

Misorientation Angle-Axis

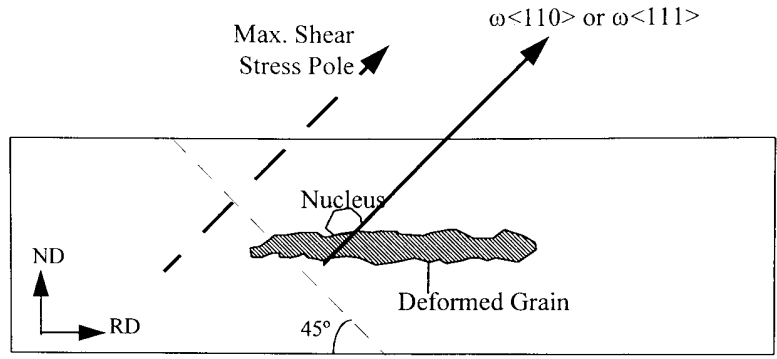

(b)

Fig. 20. (a) Geometry of 'plane matching' as it applies to deformed grain B (lower left) being consumed by new grain A (upper right). Note that the highlighted $\{110\}$ planes in grains $\mathrm{A}$ and $\mathrm{B}$ share a common $\langle 110\rangle$ normal, which lies in the boundary. The angle of rotation about this axis required to bring the grains into correspondence is the angle of misorientation between the two grains [148]. (b) Schematic representation of one of the two maximum shear stress poles associated with rolling. Also shown is a $\langle 110\rangle$ or $\langle 111\rangle$ misorientation axis that is nearly parallel to the maximum shear stress pole. The former can be used to specify the angle of misorientation associated with a particular boundary; such boundaries frequently display high mobilities (after [149]).

new or recrystallized grain can be described in terms of a rotation about a $\langle 110\rangle$ or $\langle 111\rangle$ axis that is common to both grains. Although there are six crystallographically equivalent $\langle 110\rangle$ axes (and four equivalent $\langle 111\rangle$ axes), it appears that only the axes in the vicinity of the maximum shear stress poles are able to participate in the transformation. For the case of plane strain rolling, these poles are inclined at $45^{\circ}$ to both the ND and RD directions (see Fig. 20(b)). Similar axis selection has been reported to occur during torsion testing [143].

The migration of $\langle 110\rangle$ and $\langle 111\rangle$ boundaries, but not $\langle 100\rangle$ boundaries, has been ascribed to the much lower boundary free volumes of the former two classes of boundary (see Fig. 21, [151]). The slower migration rate of $\langle 100\rangle$ boundaries, according to this interpretation, arises from the higher proportion of impurity atoms that is able to segregate to these more accommodating interfaces. Impurity segregation has also been used to account for variant selection [150]. Under rolling conditions, planar dislocation arrays are observed that are approximately perpendicular to the maximum shear stress poles. Their presence has been considered to draw segregated impurities away from the boundaries by pipe diffusion. According to this view, variant selection takes place because the other crystallographically equivalent boundaries are not oriented so as to benefit from such accelerated diffusion.

It has also been proposed that variant selection is essentially due to the internal stresses developed during deformation $[152,153]$. Although high internal stresses are produced in cold worked materials, as indicated above, much lower dislocation densities are generated during high temperature deformation, particularly in bcc metals. As a result, the high temperature internal stresses may be too low in these materials to produce variant selection. This may be why little evidence for selective growth has been observed in bcc metals to date; this is clearly a topic that needs to be followed.

\subsection{Role of twinning}

Single crystal studies [154] have revealed that twinning plays an important role in DRX in these materials. Successive generations of twins form (e.g. second, third, fourth, etc.), some of which will clearly have more growth potential (in their specific environments) than others. In a similar vein, some grain orientations may be more susceptible to twinning than others. Thus, the process of twinning can contribute to both oriented nucleation and selective growth.

The picture is slightly different in polycrystalline materials because the presence of grain boundaries makes it somewhat easier for heterogeneous nucleation to take place. Nevertheless, even in these materials, twinning can play an important role in the propagation

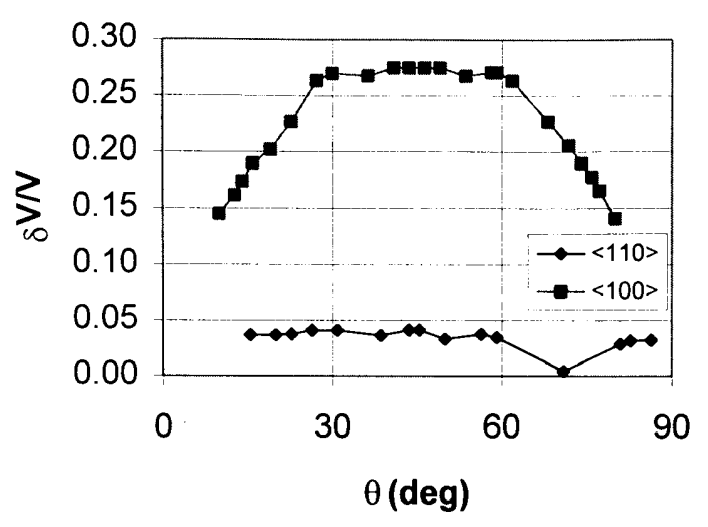

Fig. 21. Orientation dependence of the grain boundary free volume [151]. Note that high angle $\langle 100\rangle$ boundaries have approximately six or seven times the free volume of $\langle 110\rangle$ boundaries. Similar remarks apply to $\langle 111\rangle$ boundaries, although their low free volumes are not shown here. 


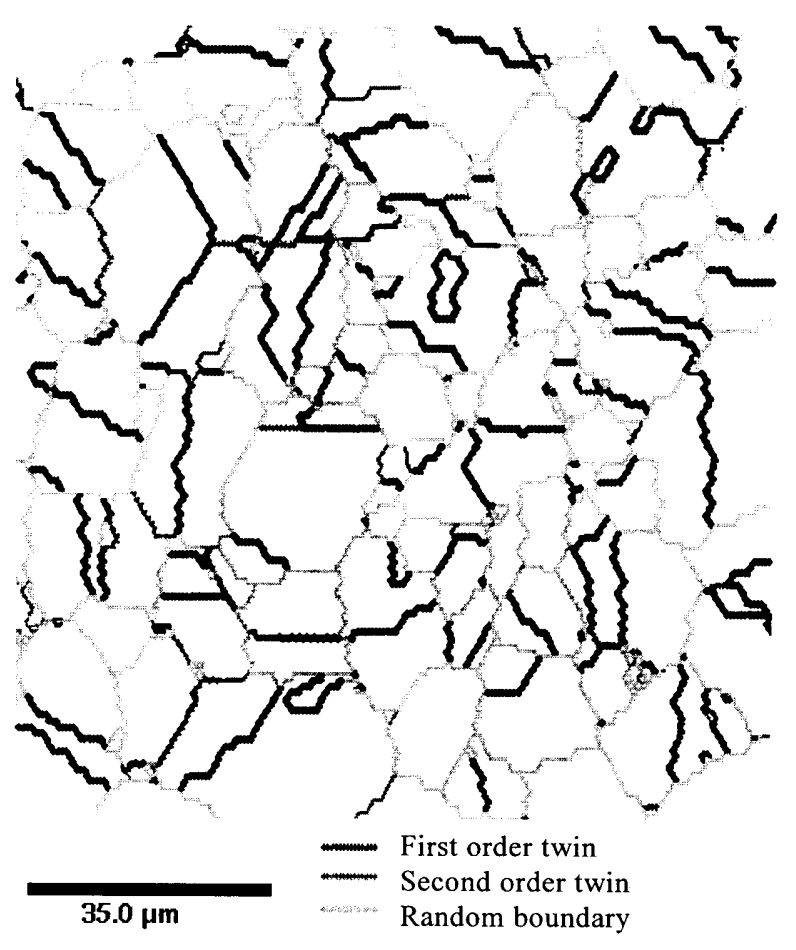

Fig. 22. Misorientation map of 304 stainless steel deformed in torsion at a strain rate of $1 \mathrm{~s}^{-1}$ at $1050^{\circ} \mathrm{C}$. A strain of 2.2 was applied, which is within the steady state regime. Most of the boundaries can be classified as first order twins, some as second order twins, and only a minority as random boundaries [155]. (P. Bocher acknowledges with gratitude the use of the OIM facilities at Carnegie-Mellon University provided by Professor B.L. Adams).

of DRX, particularly in low stacking fault energy alloys such as the 304 stainless steels. On the deformation of these materials to the vicinity of the peak strain, many fine grains form, bounded largely by first and second order twins [155]. As the strain is increased into the steady state region, the large initial grains are replaced by a fairly homogeneous microstructure, see Fig. 22 . Most of the boundaries displayed here are in motion, and it is of interest that the majority of the interfaces can be classified as first and second order twin boundaries.

\subsection{Texture development during $D R X$}

Oriented nucleation, by its nature, leads to texture change. When low stored energy nucleation is the most important DRX mechanism, the resulting texture will be dominated by the orientations of the low stored energy grains. Similar remarks apply to high stored energy nucleation or to recrystallization via twin formation. In the work carried out to date in this area at McGill University [143,144], only low stored energy nucleation has been observed. These experiments were performed in torsion and the results may not apply to other strain paths; the latter should, therefore, be investigated. If, in fact, low stored energy orientations generally become dominant during DRX, this may signify that it is the rapid consumption of high stored energy grains that leads to their eventual disappearance. An interesting question then follows regarding the possible commercial usefulness of textured materials containing high intensities of low stored energy orientations.

As indicated above, selective growth appears to be more important during the DRX of fcc as opposed to bcc metals. When this mechanism is operating, it leads to quite specific changes in texture, especially if variant selection (i.e. the choice of particular rotation axes) is involved, see Fig. 23 [143]. Here the simulations were based on a low stored energy nucleation model together with $\langle 111\rangle$ selective growth. The latter involved only the axes that were parallel to the maximum shear stress

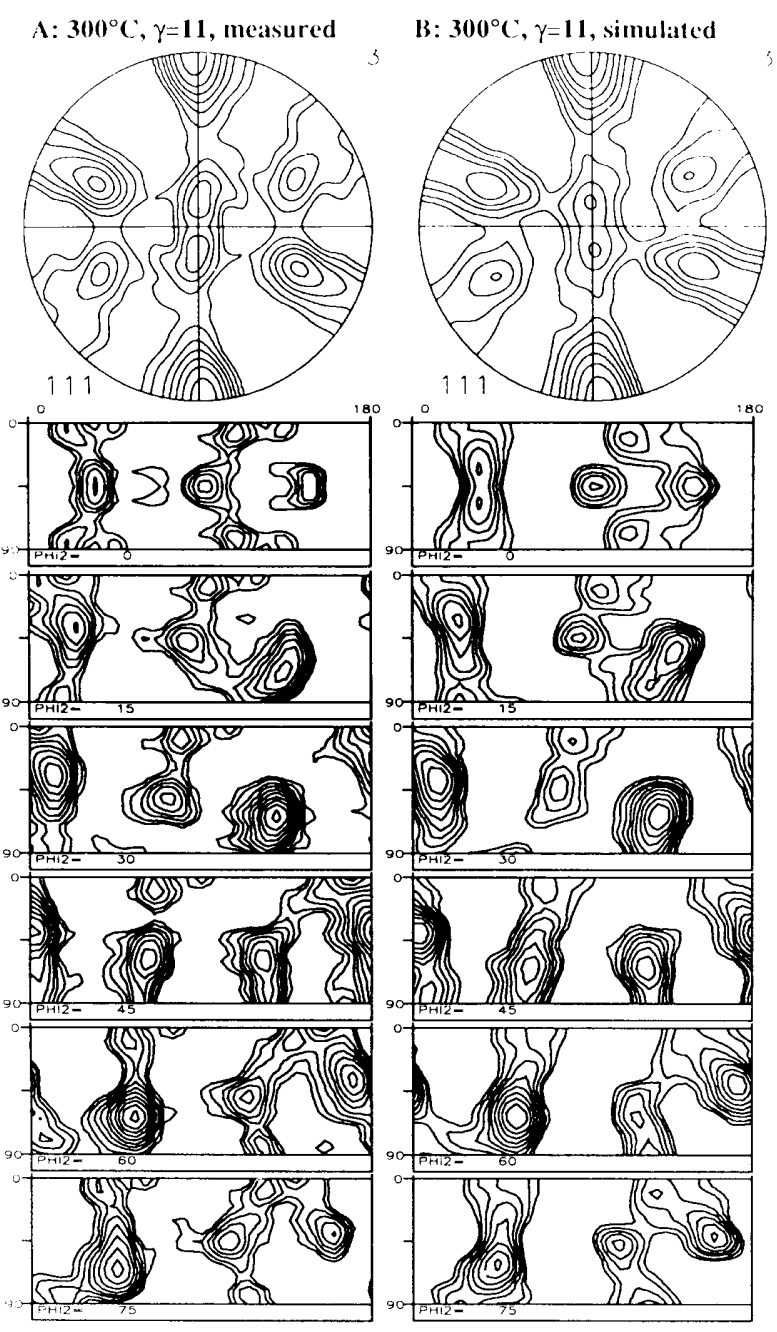

Fig. 23. (a) Measured (111) pole figure and ODF of an OFHC copper sample twisted at $300^{\circ} \mathrm{C}$ to $\gamma=11$. (b) Simulated texture starting with the experimental initial texture using crystallographic slip and dynamic recrystallization. Isovalues on all diagrams: $0.8,1.0,1.3,1.6$, $2.0,2.5,3.2,4.0,5.0,6.4$. 


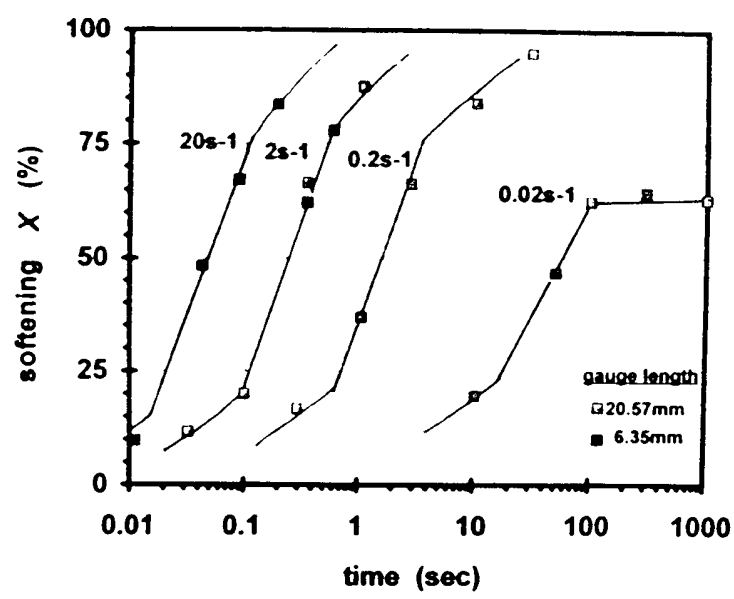

Fig. 24. Percent softening vs. log time for the metadynamic recrystallization of a plain $\mathrm{C}$ steel deformed in torsion at $950^{\circ} \mathrm{C}$ at various strain rates [157]. A strain of 0.5 was applied $\left(1.0\right.$ at $\left.20 \mathrm{~s}^{-1}\right)$, which was beyond the critical strain for the initiation of dynamic recrystallization. A preliminary 'roughing' strain of 0.5 was applied at $1 \mathrm{~s}^{-1}$ at $1050^{\circ} \mathrm{C}$ to produce a constant initial grain size of $50 \mu \mathrm{m}$.

pole (the longitudinal axis in torsion), so that variant selection played an important role. It is, therefore, important to continue to search for evidence of variant selection as well as of selective growth, and then to see if these can lead to industrial applications.

\subsection{Postdynamic recrystallization}

Once DRX has been initiated, it is followed by a combination of conventional static recrystallization (SRX) and metadynamic recrystallization (MDRX) [156]. The latter mechanism differs from SRX in a number of significant ways $[157,158]$. The first has to do with the kinetics. The rate of metadynamic recrystallization is sensitive to the prior strain rate but is relatively insensitive to the strain and temperature (see Fig. 24). Conversely, the rate of conventional recrystallization depends on the prestrain and temperature, but only slightly on the strain rate. The result is that MDRX can play an important role at relatively low finishing temperatures and short interpass times, a range where static recrystallization is too sluggish to make a contribution. This applies to processes such as finish rolling in rod mills, where the interpass time can be as short as $15 \mathrm{~ms}$ [158]. It should also be noted that MDRX leads to considerably finer product grain sizes than static recrystallization.

Of particular relevance here is that the accurate modelling of industrial hot working processes will require precise knowledge of the kinetics and microstructural effects of MDRX. Also required is an increased understanding of the kinetics and microstructural effects of the grain growth that follows SRX and MDRX.
Although the short interpass time rod mill behavior is now relatively well understood, as outlined above, the picture is less clear for intermediate strain rate processes such as hot strip rolling, where the interpass times are in the $1 \mathrm{~s}$ range. Much further work is required in this area to clarify the extent to which SRX is absent, so that strain accumulation takes place; the latter then leads to the initiation of DRX, followed as the case may be by SRX and MDRX. One of the particular limitations preventing the resolution of such issues is the difference between the strain rates in the final stands of the mill $\left(100-200 \mathrm{~s}^{-1}\right)$ and those readily attainable in laboratories $\left(5-10 \mathrm{~s}^{-1}\right)$. This difference affects the kinetics of both DRX and MDRX and thus reduces the accuracy and reliability of extrapolations from physical simulations.

\subsection{The interaction between precipitation and $D R X$}

In steels, the initiation of strain-induced precipitation has the ability to prevent or suspend recrystallization. This applies to both static and dynamic recrystallization. However, continuing deformation also leads to coarsening of the 'old' precipitates, so that 'fresh' ones are generally considered to be required for the prevention of recrystallization. In hot strip rolling, where the interpass and total process times are relatively short (e.g. 1 and $10 \mathrm{~s}$, respectively), because of the competition between precipitation and coarsening, the relative importance of 'old' and 'new' precipitates is not yet clear. This issue is again of importance with respect to the modelling of rolling operations; this is because the interruption of DRX (as well as of SRX and MDRX) leads to increases in rolling loads, whereas the initiation of DRX (and MDRX), which requires the absence of precipitation, leads to sudden decreases in rolling loads.

The effect of precipitation during straining (dynamic precipitation) on retarding the initiation of DRX is illustrated in Fig. 25(a) for a $0.018 \% \mathrm{Nb}$ steel. Here it can be seen that longer holding times prior to testing, which allow for more complete static precipitation, are responsible for reducing the peak strain. Similar tendencies can be observed in Fig. 25(b) for a $0.035 \% \mathrm{Nb}$ steel; it is evident here that the occurrence of strain-induced precipitation during testing increases the peak strain [159].

\subsection{Effect of solute elements on precipitation and on $D R X$}

As indicated above, when precipitation occurs, it prevents DRX from taking place. Now the rate of precipitation depends primarily on the 'solubility product' of the precipitating species $(\mathrm{NbCN}$ in the case of most steels). However, prediction of the precipitation kinetics on the basis of this equilibrium quantity is 
notoriously unreliable; as a result, so is that of the occurrence of DRX (and of MDRX). A factor that has recently come to light as playing an important role is the presence of non-precipitating elements that can change the 'activities' (apparent concentrations) of $\mathrm{C}$ and $N$ [160]. In this way, these elements can modify the effective solubility products.

Two elements that seem to play this role in commercial steels are $\mathrm{Mn}$ and $\mathrm{Si}$, where the former depresses and the latter raises the $\mathrm{C}$ activity. Thus, high $\mathrm{Mn}: \mathrm{Si}$ ratios promote the occurrence of DRX (and MDRX) in strip mills, whereas low $\mathrm{Mn}: \mathrm{Si}$ ratios appear to be very effective in eliminating DRX entirely

(a)
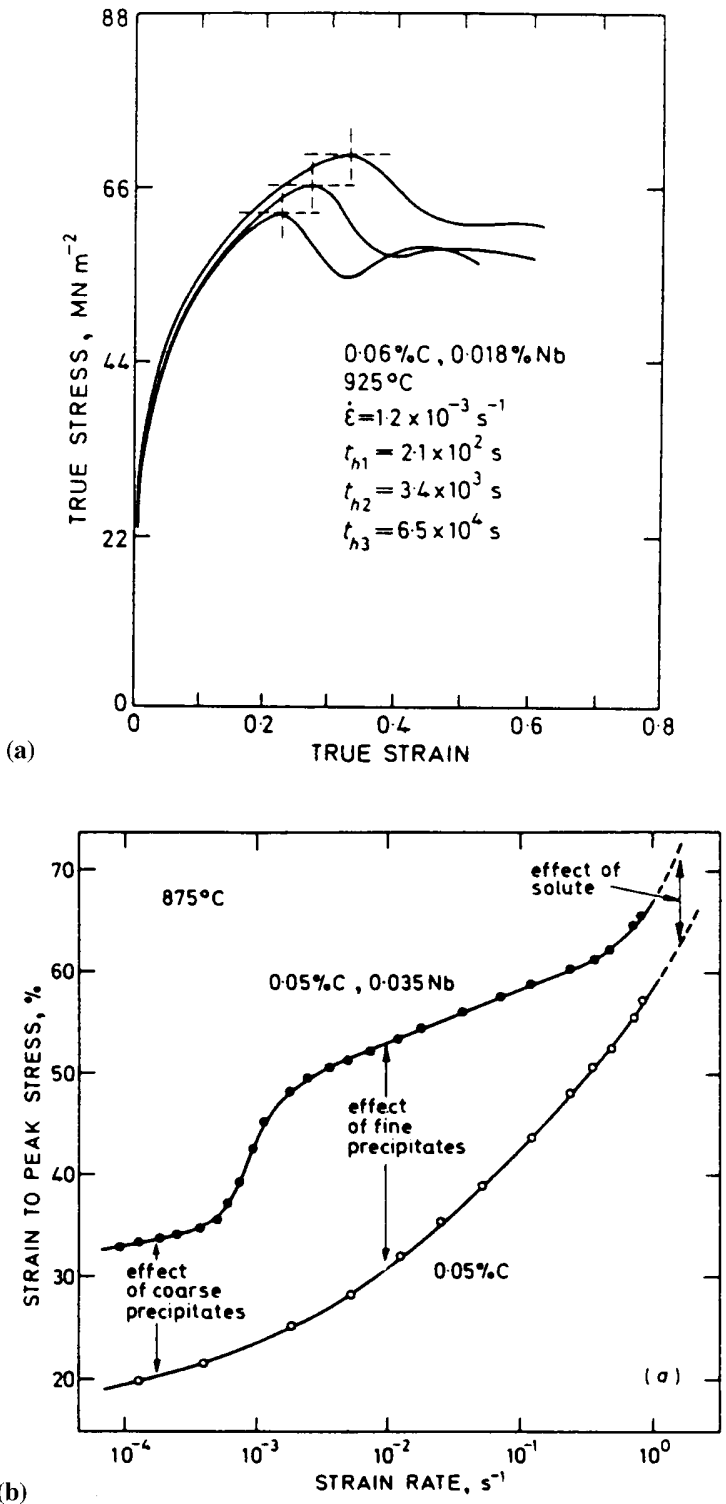

Fig. 25. (a) Effect of prior 'static' precipitation on the peak strain for dynamic recrystallization [159]. (b) Dependence of the peak strain for dynamic recrystallization on the testing strain rate [159].
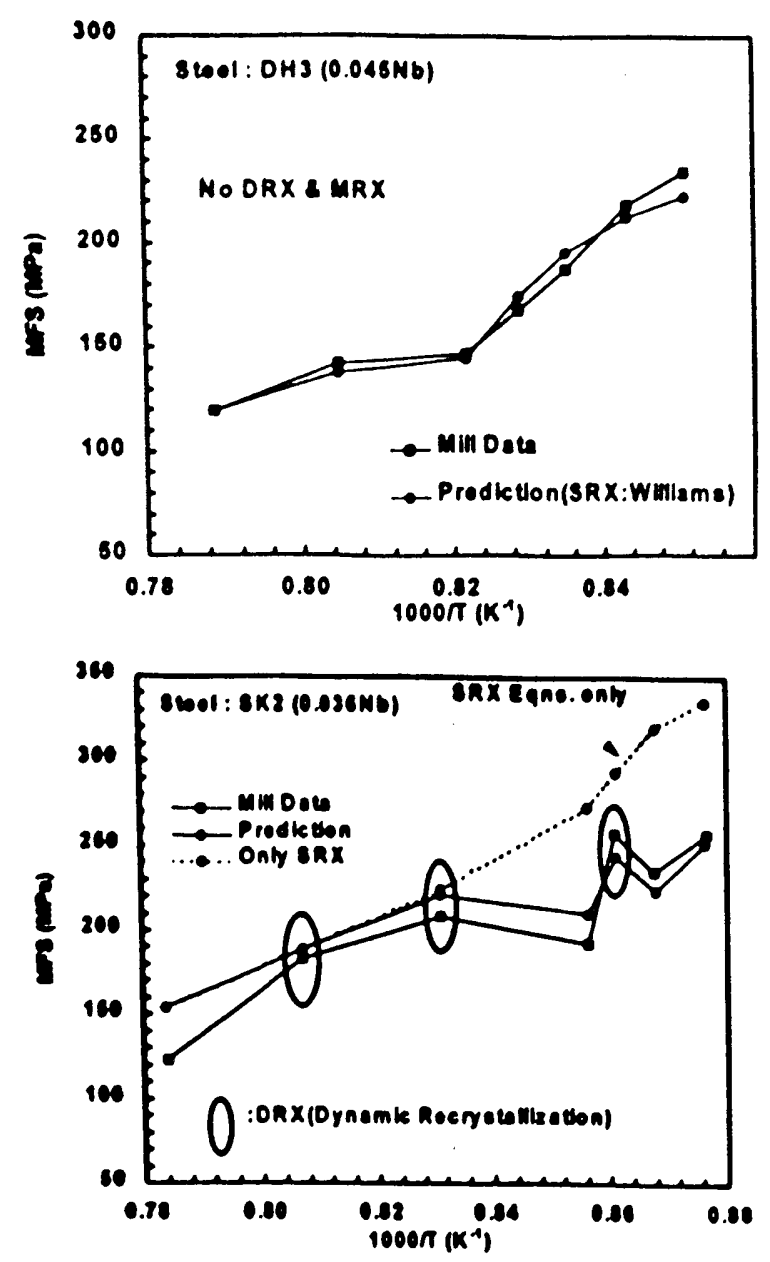

Fig. 26. Comparison of mean flow stress values measured in rolling mills with model predictions: (a) $0.045 \% \mathrm{Nb}$ steel with low $\mathrm{Mn}: \mathrm{Si}$ ratio, in which no DRX or MDRX is observed; (b) $0.036 \% \mathrm{Nb}$ steel with high $\mathrm{Mn}: \mathrm{Si}$ ratio, in which sudden load drops due to the initiation of DRX followed by MDRX are apparent (after [160]).

[160]. Because of its commercial significance, it is evident that this important effect is worth investigation in some detail. Also of interest is the effect of other alloying elements that remain in solution in austenite, such as $\mathrm{Cr}$, Mo and $\mathrm{Ni}$.

Examples of the effects of $\mathrm{Mn}$ and $\mathrm{Si}$ addition on the rapidity of $\mathrm{NbCN}$ precipitation are presented in Fig. 26. Here it can be seen that there is neither DRX or MDRX when the Mn:Si ratio is low. This has been attributed to the attendant increase in $\mathrm{C}$ activity, which leads in turn to the occurrence of precipitation within the mill, and therefore to the suppression of DRX (Fig. 26(a)). By contrast, when the Mn:Si ratio is high, the $\mathrm{C}$ activity is decreased, and the kinetics of precipitation are retarded. Under these conditions, precipitation no longer takes place within the mill, so that the initiation and propagation of DRX and MDRX are not impeded. 

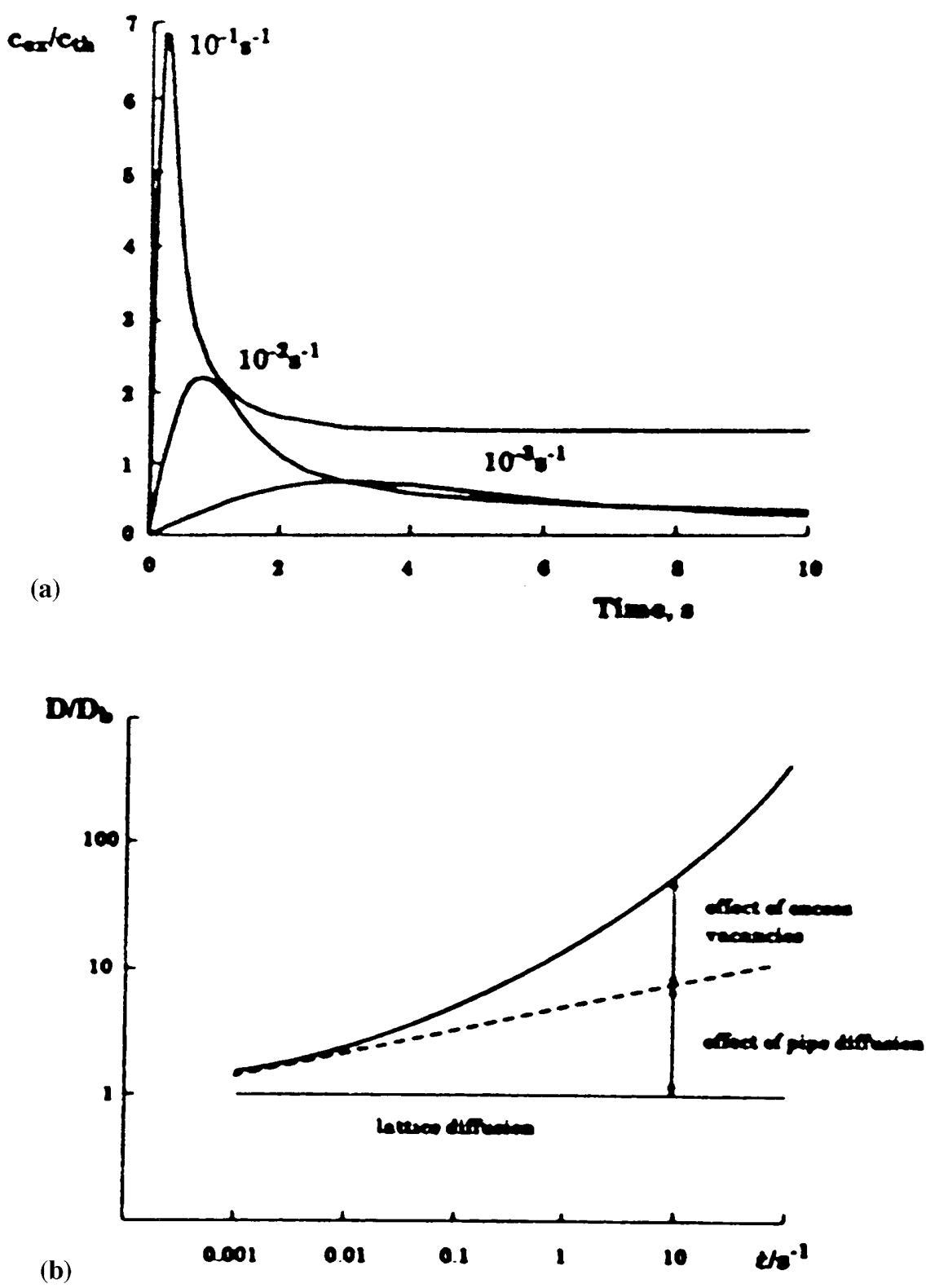

Fig. 27. (a) Calculated time and prior strain rate dependence of the excess vacancy concentration in a $\mathrm{Nb}-\mathrm{B}$ steel deformed at $1000^{\circ} \mathrm{C}[162]$. (b) Strain rate dependence of the enhancement factor for lattice self-diffusion in austenite at $1000^{\circ} \mathrm{C}$.

\subsection{Kinetics of $D R X, M D R X$ and $S R X$}

It is important, for modelling purposes, to be able to describe the kinetics of both the dynamic as well as the static softening processes in a quantitative manner. As already indicated above, DRX and MDRX are less sensitive to temperature than SRX and more sensitive to strain rate. This state of affairs has not been explained in any detail, and it has even been suggested that DRX is a type of 'athermal' process, at least in contrast to SRX [161]. The occurrence of DRX in copper shock loaded and then compressed at high strain rates at room temperature is described in the latter publication.

One factor that is expected to contribute to the relatively 'athermal' nature of DRX is the presence of the vacancies generated by ongoing deformation. These disappear fairly rapidly on the cessation of straining [162], see Fig. 27(a). Nevertheless, their presence is expected to accelerate processes such as recovery (nucleation) and grain boundary diffusion (see Fig. 27(b)). As this subject has both fundamental as well as practical implication, it appears to merit much closer attention than it has received heretofore. 


\section{Continuous reactions}

\subsection{Introduction}

It is now recognized that refined grain structures may evolve homogeneously and gradually during the annealing of deformed metals, either with or without concurrent straining, even when the heterogeneous nucleation and growth stages of primary recrystallization do not occur. Various terms have been used to describe such microstructural transformations, including 'continuous recrystallization' (the most widely used), 'in-situ recrystallization', and 'extended recovery' [132,163-167]. The details of the underlying mechanisms have remained elusive, although recovery clearly contributes to high angle boundary development throughout these transformation processes. It is also commonly observed that deformation textures sharpen and components related to the stable orientations within the prior deformation textures are retained [97,132], observations also consistent with recovery. Indeed, the apparent role of recovery and lack of distinct recrystallization fronts have led to the suggestion that these transformations should be referred to as extended recovery processes and not as recrystallization reactions [167]. However, when migration of boundaries continues as they transform to high angle character the process involved are properly identified as recrystallization reactions and the adjective continuous then refers to the homogeneous nature of the transformation. If high angle boundaries do not move, or become immobilized as they develop, then the reactions are properly termed as recovery processes.

Mechanisms proposed to explain the role of recovery in high angle boundary formation during continuous recrystallization include subgrain growth via dislocation motion [168], the development of higher angle boundaries by the merging of lower angle boundaries during subgrain coalescence $[132,163,168]$, and the increase of boundary misorientation through accumulation of dislocations into the subgrain boundaries [169]. In general, these processes have been envisioned to result in a progressive buildup of boundary misorientation during annealing, resulting in a gradual transition in boundary character and formation of high angle grain boundaries [167]. Because these processes involve short-range interactions between dislocations and subgrain boundaries, or between adjacent boundaries, they are consistent with the observations of sharpening of deformation textures and stability of deformation texture components.

\subsection{Discussion}

Many factors have been shown to influence continuous recrystallization reactions [132,163]. For example, increased prior deformation may cause the continuous processes to occur in a more uniform manner. At intermediate levels of deformation, continuous recrystallization apparently proceeds within deformation bands where the dislocation density is greater than in the surrounding material [163]. After still more prior deformation, a high dislocation density may be developed more uniformly within the microstructure, which then enables the continuous reaction to proceed more readily throughout the material. Regions of uniform orientation facilitate continuous recrystallization reactions by subgrain growth, which is equivalent to normal grain growth in that larger subgrains grow at the expense of the smaller ones [13]. The absence of orientation gradients tends to preclude primary recrystallization.

Continuous recrystallization has been observed most frequently in alloys rather than in pure metals, often at relatively lower temperatures than those associated with discontinuous, i.e. primary, recrystallization as the mechanisms of discontinuous recrystallization are more strongly hindered with decreasing temperature [170]. During annealing of a deformed, metastable solid solution at temperatures below the solvus associated with the solute, dislocation rearrangement and grain boundary migration may be affected by precipitation of the solute and coarsening of the second phase precipitates [132]. Sites that may serve to initiate a reaction front for discontinuous recrystallization include coarse precipitate particles, pre-existing high angle boundaries, and subgrains exceeding a critical size. If subgrain boundaries become pinned by precipitate particle prior to the formation of high-angle grain boundaries at such sites, adjacent subgrains may grow sufficiently to permit subgrain coarsening to become the predominant mechanism of microstructural evolution [132]. Such a process of 'in situ' subgrain growth, involving only migration of dislocation boundaries, will apparently occur with less thermal activation than required for the migration of high-angle boundaries [132,163].

If dislocations provide nucleation sites for the formation of second phase particles, an increase in dislocation density results in a corresponding increase in the rate of precipitation. When the dislocation density exceeds a critical value, the incubation period for precipitation may become so short that the conditions for a combined discontinuous recrystallization-precipitation reaction are no longer realized [132,163]. Then, simultaneous precipitation on dislocations and continuous recrystallization reactions my occur and precipitate coarsening becomes the rate-controlling process [163]. In most instances cited, continuous recrystallization has been shown to occur by such combined precipitation/ recrystallization processes and so the continuous reaction is favored by a high density of nucleation sites. Usually, only those precipitation sites which have been created by the reaction of many dislocations, rather 
than individual dislocations, are necessary for rapid nucleation of incoherent precipitates [163]. Subboundary junctions may constitute such sites in highstacking-fault energy materials. When such subboundary nodes are pinned by precipitates the substructure may become unstable if particle coarsening is more rapid than the rate of substructure coarsening. Conversely, the continuous process can go on indefinitely when solute diffusion, which controls particle coarsening, is slower than lattice self-diffusion, which controls substructure coarsening [132,163].

Recent advances in electron microscopy and computer-based diffraction analysis methods have greatly facilitated the investigation of microstructural transformations in deformed metals. Fig. 28 provides microstructural and microtextural data illustrating the evolution of grain boundary structure during annealing and deformation of as-processed SUPRAL 2004. This alloy (nominally $\mathrm{Al}-6 \mathrm{wt} . \% \mathrm{Cu}-0.4 \mathrm{wt} . \% \mathrm{Zr}$ ) has been extensively studied and reported to exhibit dynamic continuous recrystallization resulting in a microstructure sufficient to support extensive superplastic flow during elevated temperature deformation [165,166,171-173]. Of particular interest here are the effects of annealing and deformation on the low-angle $\left(0-5^{\circ}\right.$ misorientation) and moderately misoriented $\left(5-15^{\circ}\right.$ misorientation) boundaries developed during the thermomechanical processing of this material. These data were acquired by an interactive electron back-scatter pattern (EBSP) analysis method involving computer-based analysis of orientation data $[174,175]$. In Fig. 28(a) the as-processed material, coarse $\theta$-phase $\left(\mathrm{Al}_{2} \mathrm{Cu}\right)$ particles are evident. Also, orientation contrast in the back scattered electron micrograph reveals directionality in the microstructure and pancaking of the original grains due to mechanical fibering introduced during the final cold rolling stage of the processing. The orientation measurements are plotted as discrete pole figures illustrating the presence of deformation texture components; the strongest components were S, brass, and S/B in the discrete Euler plot (not shown here) for this condition. These misorientation data indicate that the as-processed material possesses a significant fraction (0.235) of low-angle and moderately misoriented boundaries. Furthermore, during data collection orientations were captured as spacing of $0.5 \mu \mathrm{m}$ while the thickness of the pancaked grains is $2-3 \mu \mathrm{m}$. Therefore, the misorientation distribution reflects mostly the deformation-induced structure.

During static annealing, orientation contrast reveals the development of a nonuniform structure with a (sub)grain size of $0.5-2 \mu \mathrm{m}$. A slight sharpening of the deformation texture without the formation of new components is apparent in the discrete pole figure, also shown in Fig. 28(b). These data were obtained in the undeformed grip section of a sample tested at $450^{\circ} \mathrm{C}$. The boundary misorientation data reveal a fraction of low angle and moderately misoriented boundaries that is almost identical to that in as-processed material. Furthermore, the bimodal character of the distribution apparent in the data for the as-processed material is still apparent after annealing, with only a slight upward shift in mean misorientation for those boundaries with misorientations in the range of $5-15^{\circ}$. This suggests that the boundaries apparent in this annealed condition represent primarily boundaries that are retained from among those developed during prior processing of the material. These misoreintation data do not support a model involving significant buildup of misorientation via coalescence or accumulation of dislocations into subgrain boundaries. The development of orientation contrast and sharpening of the texture reflect recovery processes that reduce lattice strain by elimination of dislocation structures within evolving (sub)grain interiors.

The effect of concurrent straining is to accelerate microstructure coarsening. This is shown in the data of Fig. 28(c); these data are isochronal with those of Fig. 28(b) because they were acquired in the deformed gage section of the test sample. For the strain accumulated in the region examined here, the bimodal character of the misorientation distribution is still evident. These data suggest that dynamic continuous recrystallization may be reflected in a tendency for the moderately misoriented boundaries to increase in misorientation. This may reflect a randomizing effect of deformation by grain boundary sliding, or the effect of sliding on random, high angle boundaries accompanied by coalescence of lower angle boundaries as grains coarsen. At this strain, no recrystallization texture components have become apparent, again emphasizing the role of recovery processes in the continuous reaction.

\section{Geometric dynamic recrystallization}

\subsection{Introduction}

The relatively new concept of geometric dynamic recrystallization (GRX) is now described. It was originally used to explain the observation of the formation of 'crystallites' in pure aluminum deformed to relatively large strains at elevated temperature. It has been confirmed to also occur in aluminum-magnesium alloys at conditions where classic discontinuous dynamic recrystallization was originally believed to occur. GRX may also occur in a variety of higher stacking fault energy alloys where pronounced dynamic recovery occurs. 

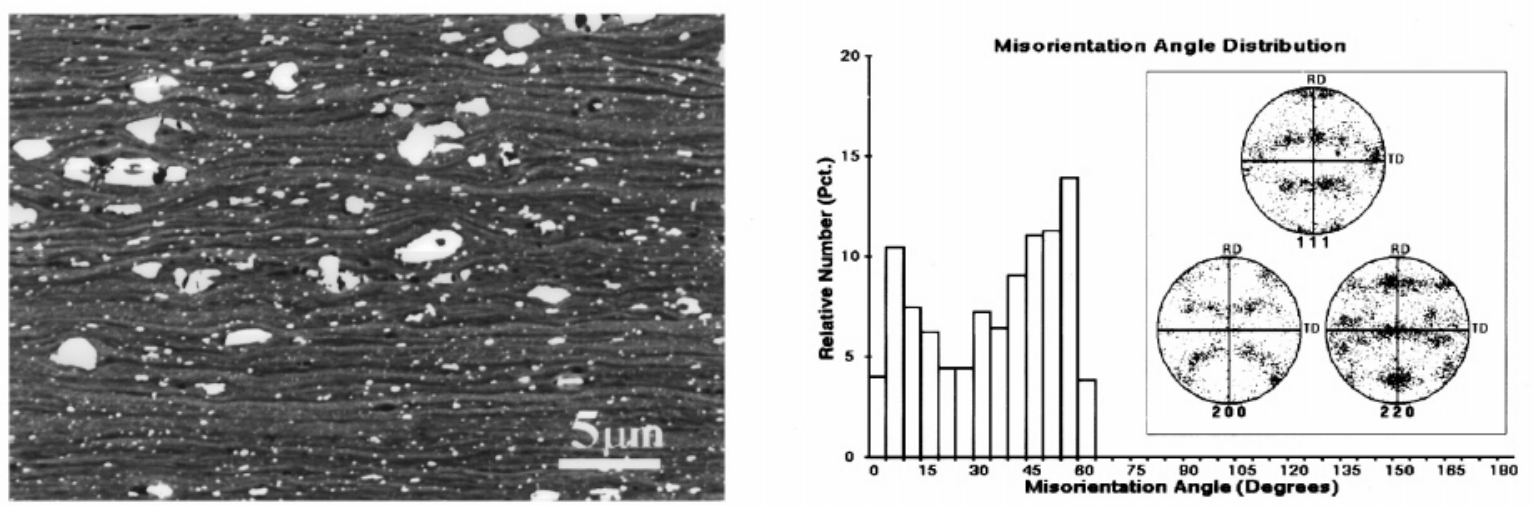

(a)
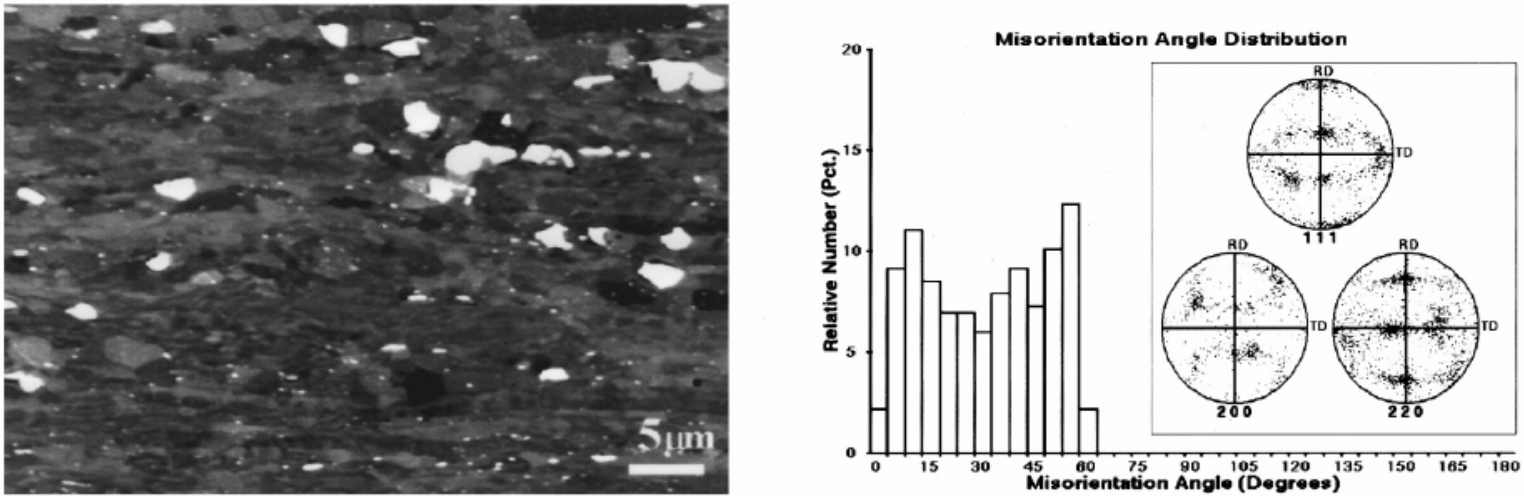

(b)
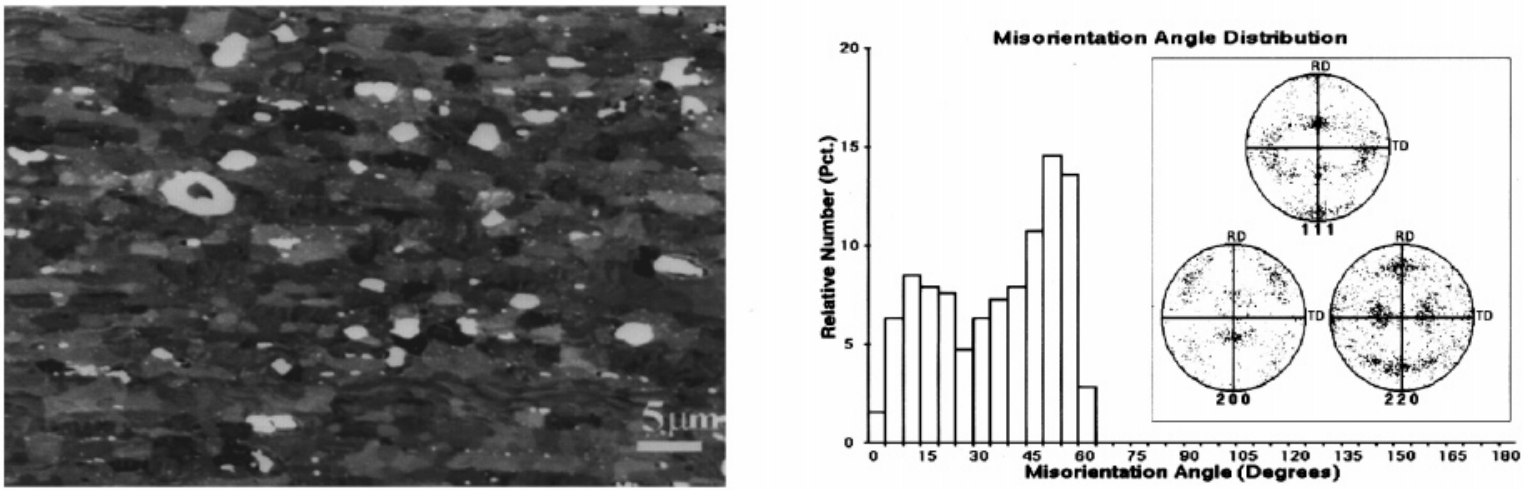

(c)

Fig. 28. Microstructural and microtextural data, in the form of back scattered electron micrographs, boundary misorientation data and discrete pole figures (as insets), showing the evolution of grain structure in SUPRAL 2004. In (a), as-processed material shows an elongated grain structure; the misorientation data are for 496 boundaries, corresponding to 497 individual orientation measurements. The undeformed grip section of a tensile sample, (b) shows a nonuniform substructure in material annealed $30 \mathrm{~min}$ at $450^{\circ} \mathrm{C}$; 317 orientation measurements provided 316 boundary misorientations. In the deformed gage section, (c) the substructure is more uniform after deformation to a strain of 0.16 at a strain rate of $10^{-2} \mathrm{~s}^{-1}$; 317 orientation measurements provided 316 boundary misorientations. Samples were examined in an electropolished (unetched) condition.

\subsection{Discussion}

The phenomenon was first described by McQueen and coworkers [176-178] who introduced the descrip- tive term 'geometric-dynamic-recrystallization'. Briefly, McQueen and coworkers discovered that very large equivalent-uniaxial (von Mises) strains (over 100) could be achieved in commercial purity aluminum at certain 
elevated temperature and strain-rates. Large ductilities of aluminum have been observed by others [179-183]. A review of these works as well as other large-strain torsional deformation work $[184,185]$ reveals the following mechanical trends.

Under constant strain-rate conditions, in torsion, the aluminum strain hardens to a broad maximum at equivalent uniaxial strains typically near two. This stress appears to be essentially the same as the steadystate stress observed in conventional tensile creep tests at the same temperature and strain-rate. Subsequently, the aluminum softens by about $20 \%$ over a range of equivalent-uniaxial strain (5-20) before reaching a stress that is independent of strain. This softening has been fairly convincingly shown to be due to texture by McQueen and coworkers [177,178] using X-ray diffraction. The observed texture implied a decrease in the average Taylor factor that is consistent with a $20 \%$ decrease in flow stress at the observed strains. Other suggestions [185-187] that this is due to substructural changes do not appear valid $[188,189]$, as will be further discussed. One interesting observation is that the activation energy for plastic flow, defined by the classic equations is the same at the peak stress as at the largest strains [187], and is approximately equal to the activation energy for lattice self-diffusion (above $0.6 T_{\mathrm{m}}$ ). This, of course, suggests that classic dislocation climb is the rate-controlling mechanism for plasticity during all stages of deformation. As will also be discussed subsequently, classic (discontinuous) dynamic recrystallization is not occurring. The dramatic ductilities observed here (as well as in some other high stacking-fault energy metals and alloys [190]) is due to pronounced dynamic recovery.

The following microstructural trends were observed. All the investigations previously referenced found, not surprisingly, that subgrains form in pure aluminum as a result of deformation. Further, all of the investigations found that the subgrains that form remain equiaxed throughout the entire deformation process. Classically, of course, subgrain boundaries have been regarded to be of relatively low misorientation (often less than $2^{\circ}$ with high-temperature creep) and form as a result of dislocation reaction. The fact that the subgrains remain relatively equiaxed and of consistent size though the ultra-large deformation suggests that the subgrain boundaries must migrate and/or annihilate to maintain the equiaxed morphology [191].

Several [176-178,184,185,189,192] but not all $[182,183]$ investigations noted that in polycrystal an increased fraction (between one-third and one-half) of the subgrain facets which were initially almost entirely of low misorientation became high angle boundaries (i.e. misorientation angle $\theta>10^{\circ}$ ) with increased strain. The phenomenon is only observed typically at strains (depending on the grain size) greater than 3 and not observed over the limited strains that can be achieved in an ordinary tensile test. Montheillet and coworkers [185] originally suggested that this observation of an increasing number of high angle boundaries was the result of the continued accumulation of dislocations in initially relatively low misorientation subgrain boundaries leading to the formation of high angle boundaries, akin to "continuous dynamic" recrystallization (see Section 7). However, such a microstructure is the result of what is now termed geometric-dynamic recrystallization (GRX).

Again, this phenomenon has also been shown to occur in aluminum magnesium alloys deformed in the viscous glide $(n=3)$ regime [193]. Briefly, for the case of torsional deformation with geometric dynamic recrystallization, the high angle subgrain facets observed after large-strain deformation are the result of the initial equiaxed grains of the starting polycrystal spiralling around the torsion axis with an accompanying reduction in axial thickness. This is well illustrated in the optical micrographs in Fig. 6 of [184], shown here in Fig. 29, and Fig. 9 of [188]. At very low plastic strains (e.g. 0.20), the high angle boundaries appear to become serrated as subgrain boundaries form. The serrations have been confirmed to consist of a triplejunction of two high angle (the serrated boundary) and a low angle subgrain boundary [184]. As the torsion specimen deforms, the serrated high angle boundaries become increasingly perpendicular to the torsion $(z)$ axis, and the separation decreases. As the grains continue to thin, the total area of high angle boundaries increases. Ultimately, the original grains thin to the point that their thickness is on the order of about twice the subgrain size. Grain boundary serration on opposite sides of the grains begin to come into contact with each other, perhaps causing the grains to 'pinch off' [194], as illustrated in Fig. 30. Annihilation of a part of the boundary results, and the high angle boundary area remains fixed with increasing strain as observed experimentally. Through this mechanism, up to one-third of the subgrain facets become high angle boundaries that have their predecessor in the original grain boundaries; they are not the result of discontinuous or continuous dynamic recrystallization. The same mechanism can occur in other deformation modes (e.g. compression [192]).

With the aluminum of Fig. 29, $d_{\mathrm{go}}=220 \mu \mathrm{m}$ and $\lambda=13 \mu \mathrm{m}$. Therefore, in torsion, the critical equivalent uniaxial strain for 'complete' GRX is about 4.9. Not surprisingly, then up to a strain of 4.05 , the original grains are still distinguishable, but beyond this strain we can no longer discern the original grains and it appears that the 'pinching off' and annihilation of high angle boundaries is occurring. 'Crystallites' appear in 


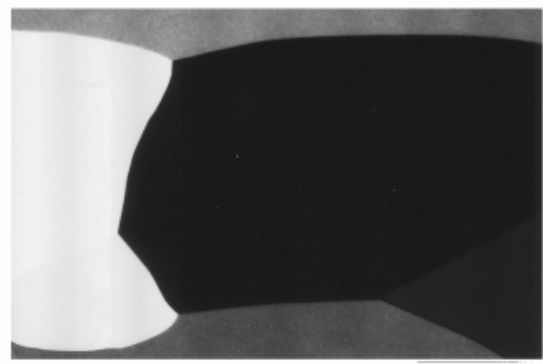

(a)

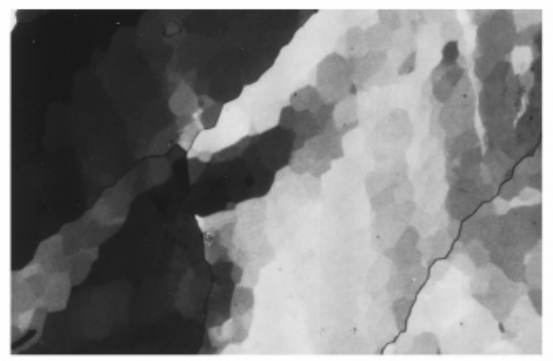

(c)

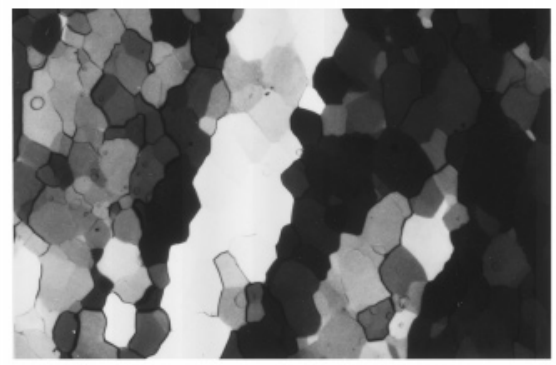

(e)

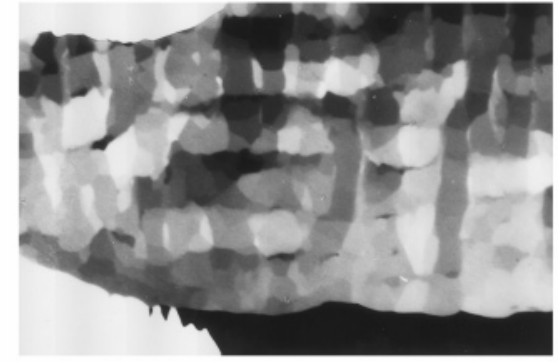

(b)

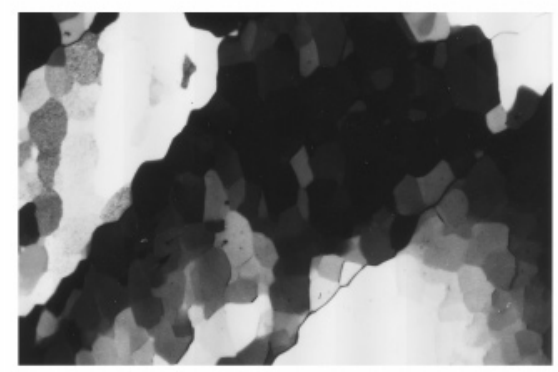

(d)

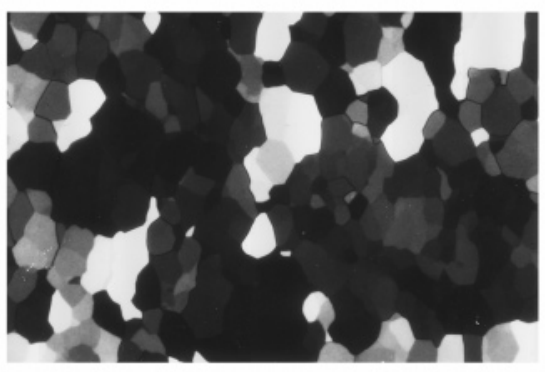

(f)

Fig. 29. High purity aluminum examined under polarized light optical metallography deformed to various strains at $\dot{\varepsilon}=5 \times 10^{-4} \mathrm{~s}-1,371^{\circ} \mathrm{C}$ : (a) 0 , (b) 0.2 , (c) 0.6 , (d) 1.26 , (e) 4.05 and (f) 16.3 .

polarized-light microscopy in which the perimeters are principally of high angle boundaries. Understandably, this structure in pure aluminum has been falsely described as resulting from classic discontinuous or continuous dynamic recrystallization.
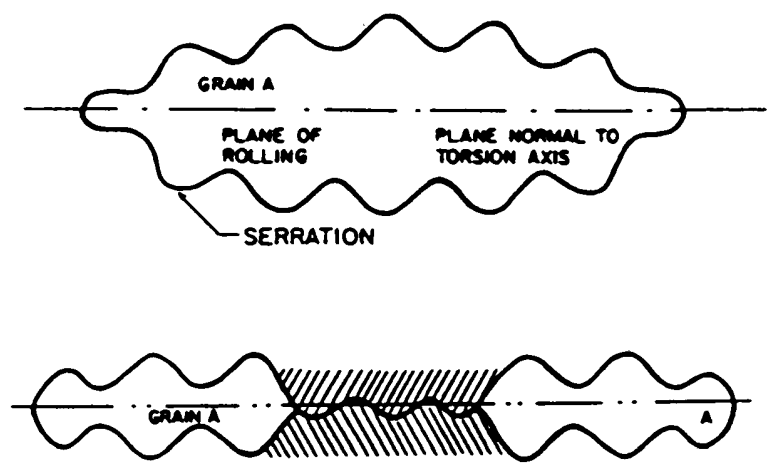

Fig. 30. When the grains are elongated and thinned extremely, they pinch off where opposite serrations meet.
There were several subsequent tests or demonstrations of this concept. The first is related to torsional deformation of aluminum single crystals at elevated temperature. If either a continuous or discontinuous dynamic-recrystallisation model is viable, then high angle boundaries would form (just as for the polycrystalline case), and about one-third or one-half of the subgrain facets would eventually become high angle boundaries. However, if GRX was operating, then only boundaries of lower misorientation might be expected to form even after very large strains. High purity aluminum crystals were aligned such that a $\langle 111\rangle$ direction was parallel to the torsion axis in [189]. The specimens were deformed at about $0.7 T_{\mathrm{m}}$ and microstructurally examined at equivalent uniaxial strains of 3.6 and 16.33. TEM analysis revealed that only about $9 \%$ of subgrain facets had misorientations greater than $10^{\circ}$. Therefore, more than three times as many high angle boundaries are observed in polycrystal than in single crystals deformed to the same large strains at elevated temperature, although TEM analysis revealed 
that the subgrain size and morphology are essentially the same. Therefore, it is believed that these results confirm that the dramatic increase in high angle boundary areas with large strain deformation of polycrystalline aluminum is substantially the result of geometric dynamic recrystallization.

Recently, Yamagata [195,196] concluded there is discontinuous dynamic recrystallization in compression tests of high purity aluminum single crystals at $260^{\circ} \mathrm{C}$, in contradiction to the work discussed above. Their conclusion appears to be based on some in-situ X-ray (Laue) diffraction studies. High angle boundaries were observed and they concluded DRX was occurring. However, it is possible that the source of the new grain boundaries is identical to that in the Kassner [189] study, which concluded these resulted from dislocation reaction induced by localized deformation. It must be mentioned, however, that ambient temperature compression of high purity $(99.999 \%$ but not $99.99 \%$ pure) results in DRX [197]. Recent analysis by Montheillet and coworkers (H.J. McQueen, private communication, 1996). suggested that some of the high angle boundaries observed in large strain deformation of aluminum at elevated temperature formed from such reactions, based on the strain for the onset of GRX being somewhat low. These investigators, however, retained the GRX concept.

Another application of the GRX concept is to the elevated temperature deformation of $\mathrm{Al}-\mathrm{Mg}$ alloys. For example, several investigators [198-201] claim to have observed classic discontinuous dynamic recrystallization in hot worked $\mathrm{Al}-\mathrm{Mg}$ alloys. A review of this work suggested that, while in some cases (higher $\mathrm{Mg}$ composition and the presence of second-phase particles) classic discontinuous dynamic recrystallization may occur, for other cases, the microstructures may have been misunderstood and GRX may have occurred [202]. To test this latter possibility, an $\mathrm{Al}-5.8 \mathrm{at} . \% \mathrm{Mg}$ alloy was deformed in torsion within the solute drag regime to various strains, up to the failure strain of 10.81 [193]. TEM revealed that subgrain formation is sluggish but that subgrains eventually $(\varepsilon=1)$ fill the grains. The steady-state subgrain size $(\lambda=6 \mu \mathrm{m})$ and average misorientation angle $\left(\varepsilon=1.6^{\circ}\right)$ are reached by $\varepsilon=2$. These observations confirm that subgrains eventually form during deformation in the solute drag regime, though they do not appear to significantly influence the strength. At low strains, nearly all of the boundaries form by dislocation reaction and are low angle $(\theta<$ $10^{\circ}$ ). At a strain of 10.81 , however, the boundary misorientation histogram is bimodal, with nearly $25 \%$ of the boundaries having high angles due to their ancestry in the original grain boundaries. This is consistent with optical microscopy observations of the elongation and thinning of the original grains as they spiral around the torsion axis. No evidence was found for classic discontinuous dynamic recrystallization. It is concluded that dynamic recovery in the solute drag regime gives rise to GRX in a manner very similar to that already established for pure aluminum, suggesting that GRX may occur generally in materials with highstacking-fault energy deformed to large strains. Recent experiments by Poschmann and McQueen [203] confirm these $\mathrm{Al}-5 \mathrm{Mg}$ results and interpretations.

There is some other evidence that GRX may occur in ferrous high-stacking-fault energy materials deformed in torsion at elevated temperature. In $\mathrm{Fe} 26 \mathrm{Cr}-1 \mathrm{Mo}$, (bcc), [204] the flow stress decreases 33\% across the strain-range $2-16$, which is greater than that in $\mathrm{Al}$; however, the change in Taylor factor with texture formation in bcc could be different from that in fcc [205]. The report indicated that no preferred orientation formed but this is likely a misinterpretation. Ambient temperature compression yield strength tests of the torsion specimens quenched to various elevated-temperature strains (compression axis identical to the torsion axis) revealed hardening at strain where torsional softening was observed. This is consistent with the development of a texture [186]. The dramatic increase (to 50\%) in high angle boundaries was concluded to be a result of continuous dynamic recrystallization. No consideration was given to the possibility that these boundaries might be those of the original grains. Finally, it should be mentioned that GRX is distinct from "rotation recrystallization" proposed by Meyers and coworkers [205] for shear bonds formed at high strain-rates.

\section{The hot worked state}

\subsection{Introduction}

The microstructures produced by hot working are explained by initially considering an ideal state that was conceived originally from creep research and has been continually modified to reflect the increasing knowledge of dislocation arrangements. It is pointed out that the structures observed in ideal hot working tests, i.e. constant temperature $T$ and strain rate $\dot{\varepsilon}$ (some $10^{4}$ faster than creep) to large uniform strains, are consistent with those of tensile creep to modest strains. From this model, the real hot worked structure diverges due to polycrystal constraints, albeit reduced by high $T$ deformation mechanisms, that give rise to persistent deformation bands of high misorientation $\theta$. From such high $\theta$ features, the possible structures are further altered by static recrystallization in multistage processing or by dynamic recrystallization in low stacking fault energy alloys. The transition from hot to cold worked state during warm working complete the discussion. 


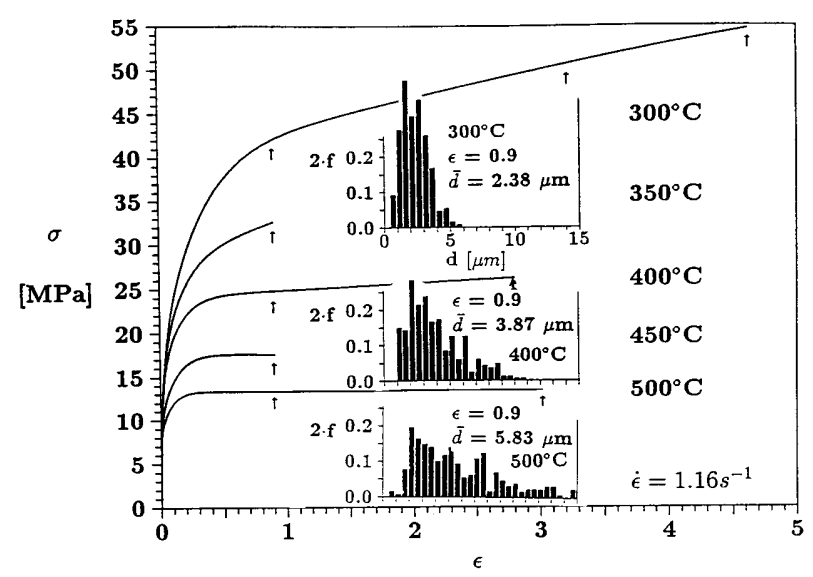

Fig. 31. Torsion flow curves for $\mathrm{Al}$ including the TEM subgrain sizes developed; the distributions are log normal with increasing numbers of larger cells at higher $T[213,214]$.

\subsection{The ideal state}

High temperature creep has been the subject of numerous dislocation climb theories related to the power law and activation energy $Q_{\mathrm{c}}\left(\mathrm{kJ} \mathrm{mol}^{-1}\right)$ similar to that for self diffusion [206-212]:

$A \sigma^{n}=\dot{\varepsilon}\left(\exp \left(Q_{\mathrm{c}} / R T\right)=Z\right.$

where $A$ is a constant, $n$ stress exponent, $R$ gas constant, and $Z$ the Zener Hollomon parameter. For constant stress $\sigma$ and temperature $T$, the strain rate $\dot{\varepsilon}$ reaches a minimum stable value. In hot working, at constant $T, \dot{\varepsilon}$, and $Z$, the stress attains a steady state value $\sigma_{\mathrm{s}}$ at a strain $\varepsilon_{\mathrm{s}}$ (although there may be a decline due to deformation heating) (Fig. 31) [215-220]. While this theory applies admirably to most metals above $0.6 T_{\mathrm{m}}$ (melting, $\mathrm{K}$ ) for creep at $10^{-8}-10^{-3} \mathrm{~s}^{-1}$ and for hot working at $10^{-3}-10^{2} \mathrm{~s}^{-1}$, a more suitable stress function for hot working is either the exponential or sinh law but with a $Q_{\mathrm{HW}}$ of similar value to $Q_{\mathrm{c}}$. The low flow stresses controllable by $T$ and $\dot{\varepsilon}$ are associated with a low density dislocation substructure in a deformation mechanism which has inherently high ductility; these features explain the use of high strain hot forming operation for $80 \%$ of all metal products [216,221].

Extensive microstructural studies by X-rays, optical and electron microscopy with selected diffraction determined that subgrains develop by the by the inception of steady state (Fig. 32) [193,194,203,213-221,224-236]. They are characterized by diameter $\lambda$, an interior dislocation spacing $\rho_{\mathrm{i}}^{-0.5}$ and a wall spacing $s$ (related to misorientation $\theta$ ) which were stably tied to deformation conditions:

$$
\begin{aligned}
& \lambda=a+b \log Z \\
& \sigma=c+e(\lambda)^{-1}
\end{aligned}
$$

where $a, b, c$, and $e$ are constants. Moreover, for both creep and hot working, the above spacings have been uniquely related to the modulus normalized stress (Fig. 33) $[206,210,224-227,231,236]$. In association with the mechanical theories, this behavior is related to the climb and cross slip of the dislocations, which lead to annihilations and the arrangement of the remainder into highly polygonized subgrains; this entire mechanism is called dynamic recovery (DRV).

The fundamental relationship of high temperature deformation characteristics to the substructure are not fully resolved. The classical theories place great importance on the Frank dislocation network within the subgrains [193,207-212]. Recent theories include all of the measured features of the substructure mentioned above [206,210,227,236,238,239]. The ambiguity continues since both theories are equally applicable to the mechanical properties because of the unique relationship of subgrain size and wall character to internal network density in steady state deformation.

Hot working research confirmed the above microstructural relationships up to very high strains $(\varepsilon=$ 60) with the further significant feature that the subgrains remained equiaxed while the grains elongated [177,178,184,188,193,194,213,214,218,224-228]. Moreover, absorption of the subgrain boundaries (SGB) causes the grain boundaries (GB) to migrate locally; the resulting serrations have wavelength of about $2 \lambda$, and amplitude of $\lambda / 2$ for Al (Figs. 29 and 34) [226,231,241244]. The latter may be much higher for $\mathrm{Al}-5 \mathrm{Mg}$, leading to highly meandering GB susceptible to pinching-off $[225,226,242,243]$. When the elongating grain thickness approaches about $2 \lambda$ (dependent on $T, \dot{\varepsilon}$ ), some opposite GB serrations may meet causing pinching-off (2D view) with shortening of the grains but no further thinning (Fig. 35) [177,178,184,188,218,225227,231,232]. This pseudo dynamic recrystallization (DRX) (no nucleation or growth) has been labelled geometric, i.e. GRX, although it has also been termed grain-refining DRV (longitudinal subdivision of elongated grains enclosing constant diameter subgrains).

From the foregoing evidence, it is clear that the SGB are not permanent features like the GB but rearrange to maintain equiaxed cellular substructure $[164,177,178$, $184,188,203,218,224-228,213,214,235]$. This comes about by their migration, merging, and annihilation with reformation normal to the elongation direction; unravelling as dislocations are pulled out and reknitting in new locations are also involved. In addition to observations upon serial straining, this theory has been confirmed by HVEM of thick foils and surface analysis of SGB $[210,212,225,236]$. This repolygonization is such that the substructure is completely rearranged in a strain interval equalling the initial transient $[225,236]$. This is confirmed in tests with sudden change in $T$ or $\dot{\varepsilon}$ [220]. Reduction in subgrain size is rapid since 

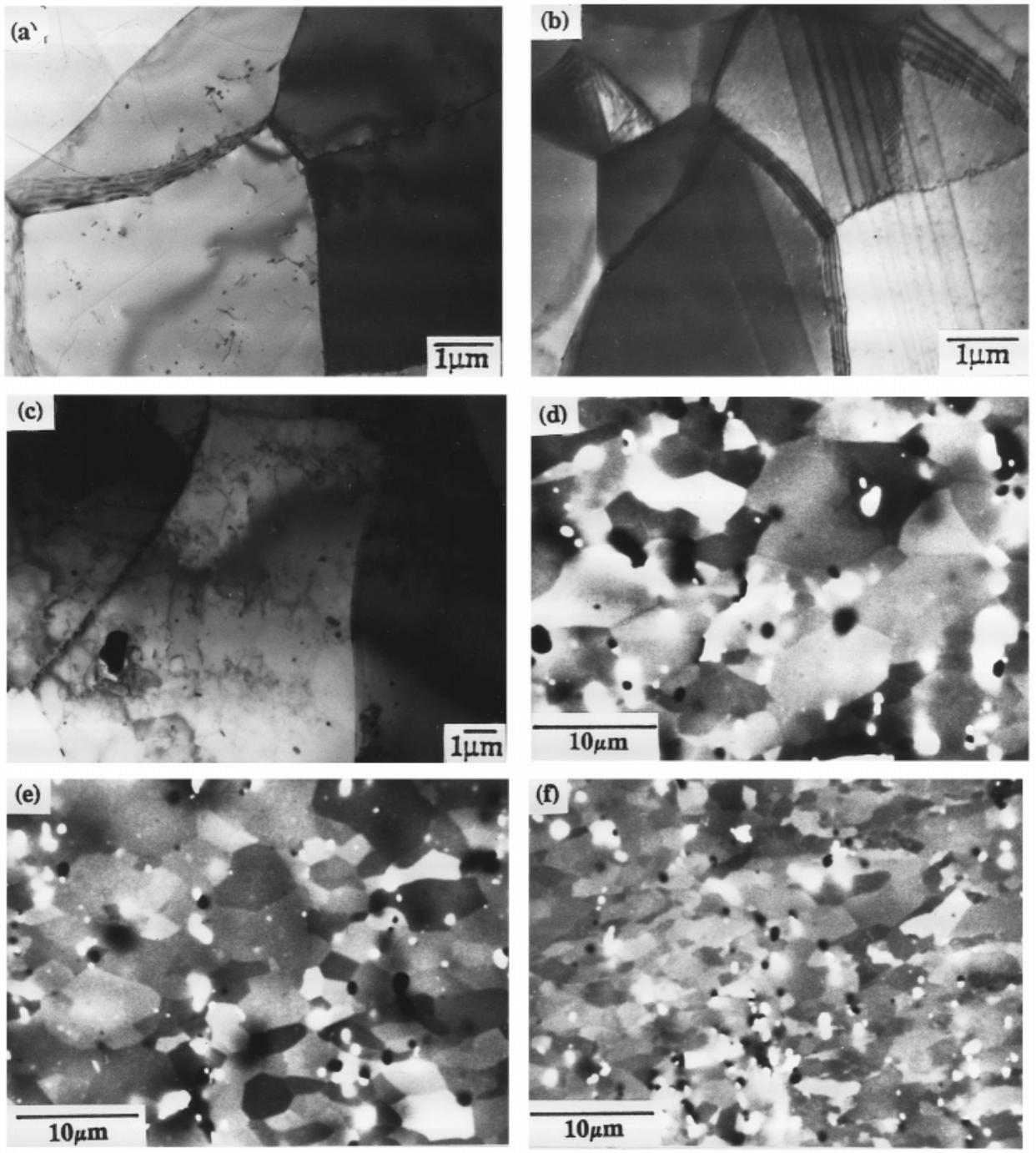

Fig. 32. TEM (a,b,c) and SEM-EBSI (d,e,f) micrographs for Al. (a) Torsion $=0.9,400^{\circ} \mathrm{C}, 1.2 \mathrm{~s}^{-1}$ related to Fig. 8-1 [213]; (b) extrusion $\varepsilon=3.4$ $350^{\circ} \mathrm{C}, 1.1 \mathrm{~s}^{-1}$ (slip traces bend about $2^{\circ}$ at SGB); (c) torsion $\varepsilon=30,400^{\circ} \mathrm{C}, 1 \mathrm{~s}^{-1}[9-18,7,19-38]$; (d,e,f) compression to $\varepsilon=-0.7$ followed by SRV at $200^{\circ} \mathrm{C}, 100 \mathrm{~h}$; (d) $400^{\circ} \mathrm{C}, 1.4 \mathrm{~s}^{-1}$ (as worked $2.4 \mu \mathrm{m}$, annealed $2.45 \mu \mathrm{m}$ ); (e) $400^{\circ} \mathrm{C}, 11.7 \mathrm{~s}^{-1}(2.0,2.4 \mu \mathrm{m})$; and (f) $200^{\circ} \mathrm{C}, 1.3 \mathrm{~s}^{-1}(1.3$, $1.7 \mu \mathrm{m})[222,223]$.

it requires creation of walls, whereas the opposite is slow requiring their annihilation. The continual rearrangement of the SGB maintains at low value (and independent of strain) the wall dislocation spacing and misorientation $\quad\left(<5^{\circ}\right) \quad[177,178,184,193,210,222-$ $227,245]$, although this is disputed [185,228]. However, permanent disorientation bands which increase in misorientation with rising $\varepsilon$ have been observed $[177,178,189,228]$. The rate of DRV at a given dislocation density is much greater under an applied stress than in the absence of stress during static recovery SRV.

In hot working there are other mechanisms that contribute to the strain, notably GB sliding and diffusion transport also associated with GBs. However, their fraction declines with rising $\dot{\varepsilon}$ to reach only 1 or $2 \%$ at
$10 \mathrm{~s}^{-1}$. GB sliding is usually associated with lattice slip and formation of dislocation density gradients which lead to sudden GB migrations [246]. In coarse grained specimens, this can cause rate surges during creep. Differential GB sliding causes w-cracking at triple junctions leading to failure in hot working unless accommodation by DRV enhanced lattice slip is able to relax the stresses $[216,218,219]$. Diffusion creep leads to atoms being deposited at GB under tension and removed from GB under compression, as well as from pores at GB under tension leading to fracture in creep. These mechanisms also reduce the constraints between the grains as explained later.

There are many conditions of high temperature deformation under which these ideal mechanisms alter towards those found in cold working. The simplest 


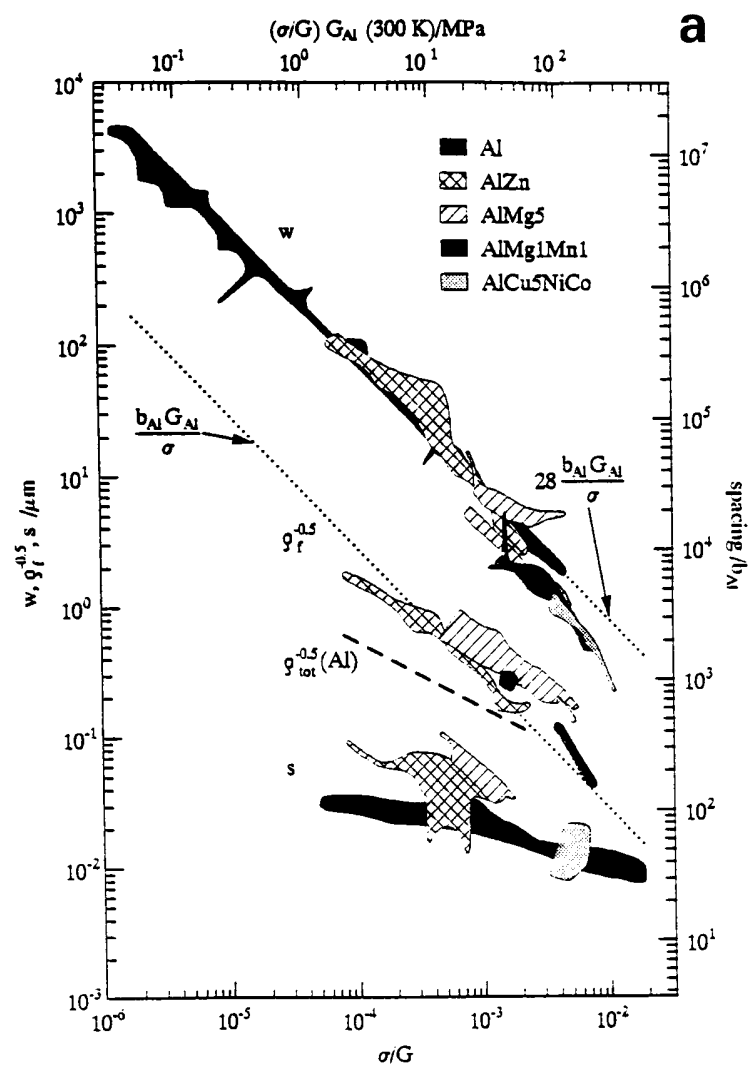

a

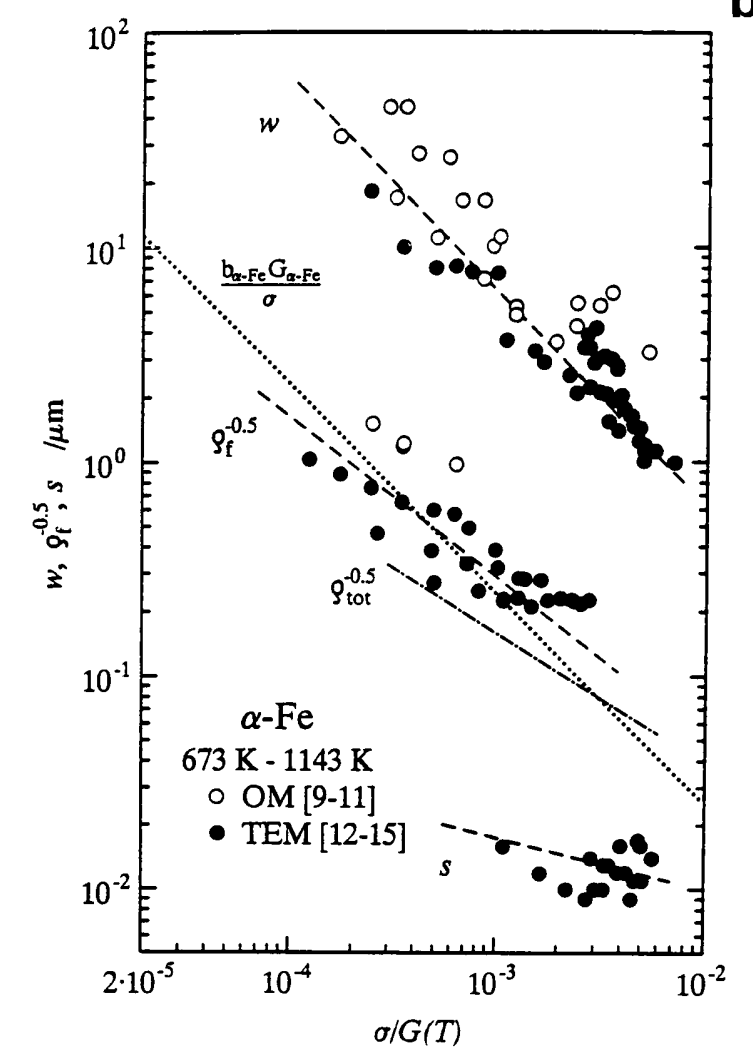

Fig. 33. The three spacings, subboundaries $\lambda(=\mathrm{w})$, internal dislocations $\rho^{-0.5}$, and wall dislocations $s$, are approximately multiples of the reciprocal of the modulus normalized stress: (a) aluminum alloys with both particles and solute; and (b) ferritic alloys. These cover a wide range of strains and strain rates in creep and hot working [237].

causes are decrease in $T$ and increase in $\dot{\varepsilon}$ which will be addressed in the discussion on warm working. The other causes are related to materials, the most significant being the stack fault energy (SFE) which give rise to DRX to be discussed after textures and multistage hot processing. Solutes and particles in increasing concentration decrease DRV, moving the alloy towards cold working behavior relative to the pure crystal.

\subsection{Polycrystal constraints}

According to Taylor analysis, the deformation of a grain in a polycrystal subjected to an arbitrary shape change would require five slip systems. This can be relaxed if the grain divides into regions each slipping on two to four systems; the scheme of division is partly defined by the deformation in neighboring grains $[37,42,55,247,248]$. These regions, deformation bands, form at low strains and begin to rotate in various directions developing boundaries called disorientation bands that increase in $\theta$ as $\varepsilon$ rises [42]. The effect is similar to that in cold working except that the constraints are reduced due to GB sliding, migrations, and vacancy flows; in consequence, there are only two or three bands per grain of $\sim 100 \mu \mathrm{m}$, much less than in cold work [236]. Moreover, the geometry of flow within the grain is relaxed by the occurrence of climb and cross slip; furthermore, extraordinary slip takes place such as on non-octahedral planes in fcc crystals $[66,212,249,250]$. On the other hand, evidence of similarities can be found in the formation of like textures in hot and cold forming [236,249,251], even though the distribution of components between grains are different $[249,250]$.

Texture formation in creep has not been of much concern because the strains are very small. In hot extrusion of Al alloys, where there are high strains without DRX or SRX, strong textures are developed. At low $T$, the $\langle 111\rangle$ component is stronger, more highly deformed, and easier to recrystallize to $\langle 111\rangle$; at high $T$, the $\langle 100\rangle$ component is stronger and less distorted [215,252,253], as confirmed be TEM-SADP [254]. These improve the service properties if they are not altered by SRX, before cooling or during solution treatment. For hot torsion up to $\varepsilon<10$, the texture is a combination of $\{1 \overline{1} \overline{1}\}[110](1 \overline{1} 2)$ and $\{001\}[110](1 \overline{1} 0)$ \{shear plane equivalent to rolling plane\}[tangent direction equivalent to rolling direction](tangent plane) in 


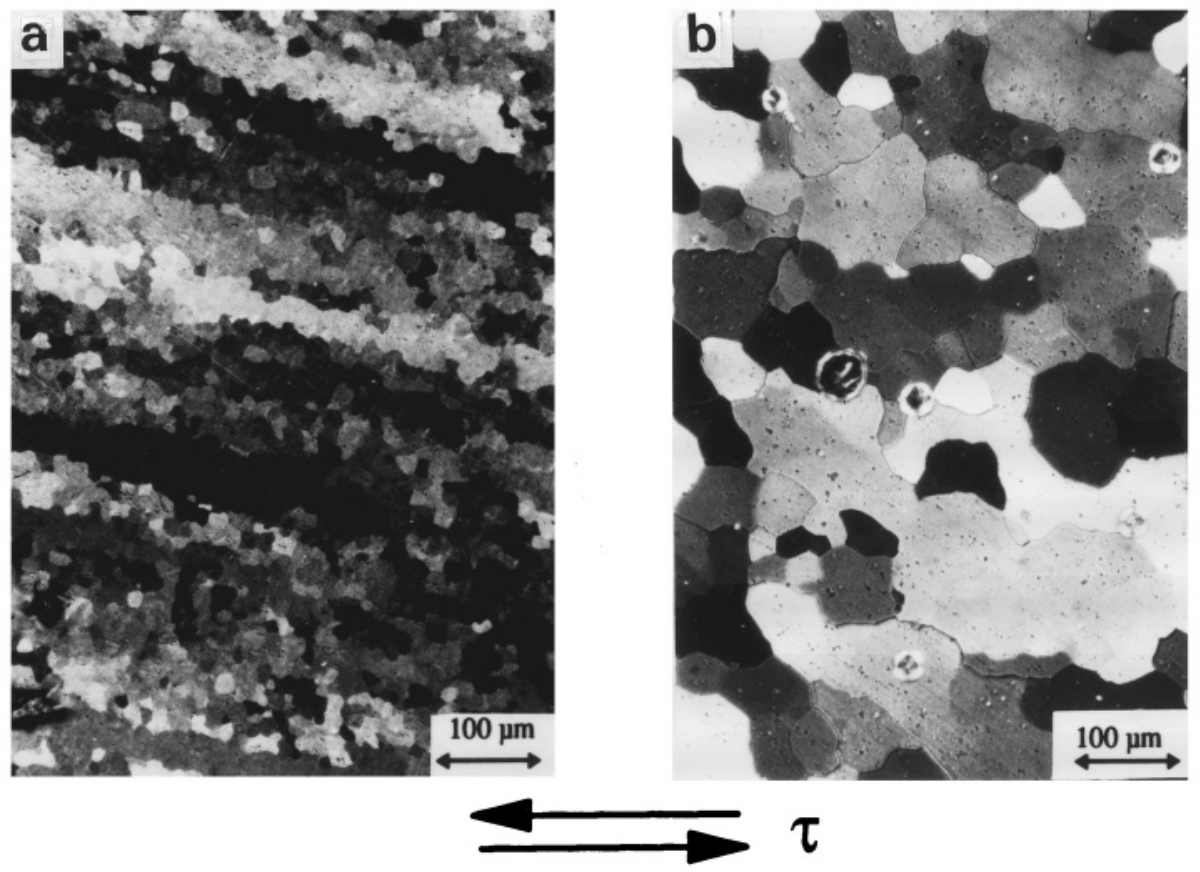

Fig. 34. Polarized optical micrographs of $\mathrm{Al}-5 \mathrm{Mg}$ in the solute drag regime showing substructure regions that may contain many subgrains as measured in TEM. (a) $400^{\circ} \mathrm{C}, 0.01 \mathrm{~s}^{-1}, \varepsilon=3.1$ exhibits elongated grains with visible subgrains and serrations; (b) $500^{\circ} \mathrm{C}, 0.01 \mathrm{~s}-1, \varepsilon=0.8$ exhibits shortened grains with thickness of approximately the subgrain diameter as a result of GRX (a). the microstructure remains almost identical to this (in b) as the strain progresses to 3.4 without change in stress [231,240] (courtesy of R. Merkel).

agreement with Taylor analysis $[177,178,185,255]$. However, at $\varepsilon>10$, a new component $\{\overline{1} 12\}[110](1 \overline{1} 1)$ develops due to the pancaked grains; the above were determined by both X-rays and STEM-SADP $[177,178,248]$. In hot rolling with avoidance of SRX, which is difficult since it requires large passes without intermediate stages, the texture in $\mathrm{Al}$ appears similar to that at $25^{\circ} \mathrm{C}$. In 304 stainless steel, the texture above $800^{\circ} \mathrm{C}$ is much more similar to that of $\mathrm{Cu}$, in contrast to that below $400^{\circ} \mathrm{C}$ which is like brass [256,257]. Upon recrystallization after hot rolling with the above textures, the cube texture develops as in SRX after cold rolling. However, hot rolling with cold rolls always causes some chilling so that the conditions are more realistically classified as warm $[251,258]$.

Additional analysis (STEM-SADP) of hot deformation microstructures with rising $\varepsilon$ indicates that deformation bands form containing the subgrains described earlier $[177,178,189,228]$. In TEM, the disorientation bands appear to be narrow boundaries which are similar to normal SGB but can only be identified by SADP. Unlike the SGB, however, they are permanent features which increase in misorientation and align parallel to the elongated GB with rising $\varepsilon[225,236]$. The deformation bands do not subdivide into blocks as in cold working $[37,247]$ for several reasons. The internal constraints are reduced by cross slip, climb, and novel slip planes. Moreover, the SGB are completely rearranged within a short strain interval; this permits elimination of old dislocations when new slip systems are introduced by rotation of the band [225,236,259-261]. Another important difference from cold working is the scale of the cellular structure; in hot working it is five to ten times larger so that the potential blocks would be of the scale of the deformation bands. Furthermore, an important mechanism of block subdivision, microband formation, is not observed in hot deformation.

\subsection{Multistage processing}

Hot rolling, which is the highest tonnage industrial mechanical forming process, is usually achieved in multiple passes with intervals between, which permit static recovery (SRV) [220,262] or recrystallization (SRX) if $\varepsilon>\varepsilon_{\mathrm{CS}}$ (Fig. 36) $[216,218,229,239]$. The first has limited effect on the developing texture whereas the second has a marked effect. The behaviours of these two mechanisms after a hot forming stage are examined partly to clarify the nature of the hot worked state and to show how they affect its complete development.

Despite the high level of DRV at the end of a hot working pass, SRV proceeds at a rate consistent with the dislocation density or the flow stress $[216,218,220,267]$. Initially, the dislocation density diminishes in the interior and in the SGB and finally enlargement of the subgrains follows [262]. However, this static process heterogeneously develops enlarged regions with high misorientations which can grow as 
recrystallization nuclei [267]. It is remarkable that the incubation period to produce SRX is short compared with the time interval $(\varepsilon / \dot{\varepsilon})$ during which steady state straining can proceed without recrystallization. When a further pass occurs at constant $T$ after SRV alone, the flow stress is reduced initially compared with instantaneous reloading but it may finally regain the uninterrupted value $[229,268-270]$. When temperature decreases across the schedule, the interpass SRV has an effect equivalent to deformation at a higher average temperature with an equivalent effect on texture.

If the interval duration is long enough, SRX can take place, especially at higher $T$ and usually results in grain refinement and elimination of the weak rolling texture developed [263-266,268,271]. For higher $T$ deformation but fixed holding temperature, the rate of SRX and
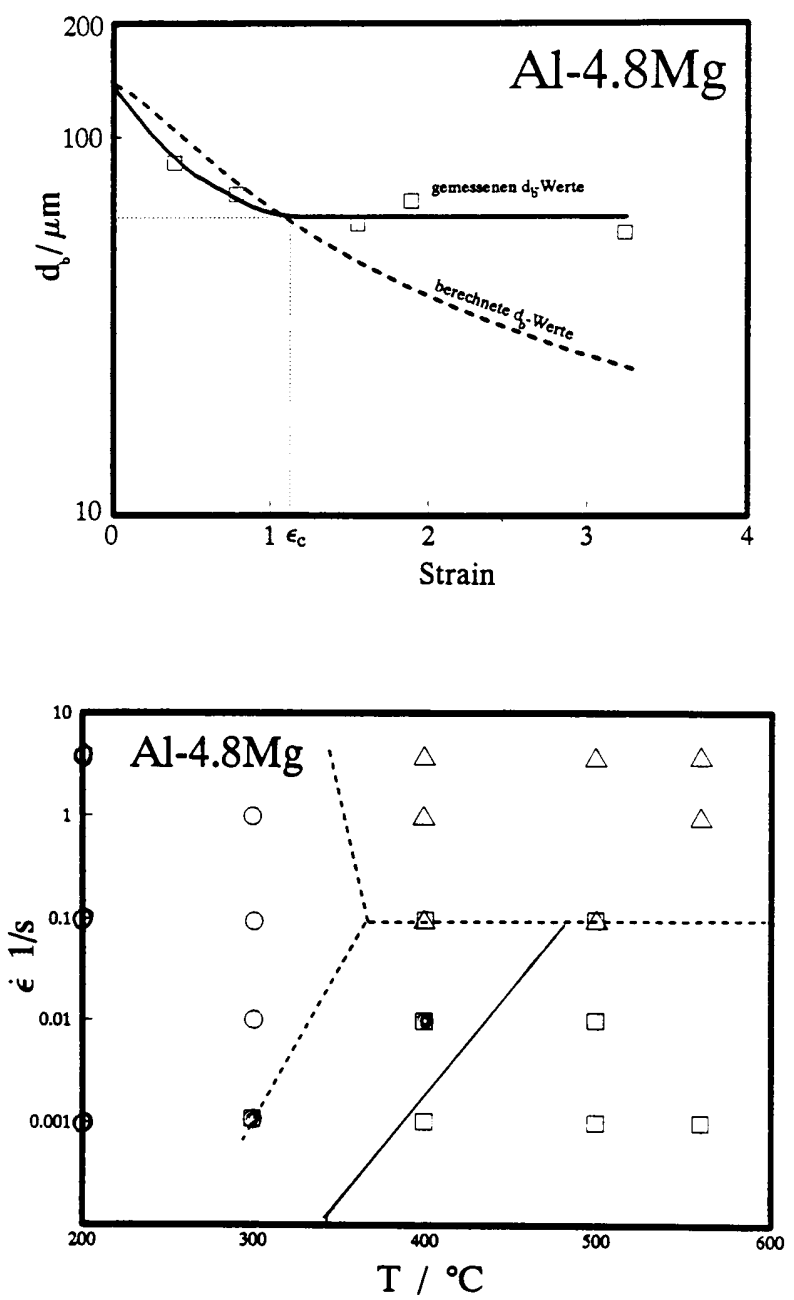

Fig. 35. The quantitative evidence for GRX appears in this stabilization of the grain thickness at $\varepsilon>1.2$ while the calculated value declines and in the microstructural $\dot{\varepsilon}-T$ map where the solid line boundary is calculated by equating the grain thickness from plasticity analysis to the subgrain size derived from the spacing graph (Fig. 33). Circles indicate elongated grains, squares condition for GRX, and triangles occurrence of SRX [240] (courtesy of R. Merkel). the number of nuclei are lower, reflecting the decreased stress level and the more highly recovered substructure (larger $\lambda$ ). In an industrial schedule with decreasing temperature, physical simulation shows that the fractions recrystallized, in constant intervals following equal passes, decrease $[216,229,271,268,263-266]$. Thus, increasing volume fractions of deformed but slightly recovered material are carried into succeeding passes raising the flow stress. If SRX becomes completely absent, the strain builds up across several passes and may initiate DRX; it is possible that nucleation starts during an interval and growth continues dynamically [265,272]. In controlled rolling of C or HSLA steels, the objective is to utilize SRX for grain refinement after the initial few passes but after intermediate cooling to prevent it in the finishing passes in order to pancake the grains leading to high nucleation rates for transformation into fine ferrite $[221,273,274]$.

\subsection{Dynamic recrystallization}

With metals and alloys of low SFE, such as Ni, $\gamma$-Fe, and $\mathrm{Cu}$, the ideal steady state DRV level is attained only in low $\dot{\varepsilon}$ creep; rate surges are found in high $\sigma$ creep. In hot working, a peak in the flow curve followed by softening to a new steady state regime is indicative of DRX. The level of DRV is inadequate to balance the stain hardening accumulation of dislocations so that the substructure reaches a density (at a critical strain $\varepsilon_{\mathrm{CD}}$ ), which supports nucleation, causing first a marked reduction in strain hardening to create a peak and then flow softening [142,216,217,221, 233,267,275-283]. (The occurrence of multiple peaks when DRX results in grain coarsening will not be discussed [142]). Before completion of the first cycle of DRX, the new grains have been deformed and attain a condition suitable for another cycle of nucleation [276,278-283]. The steady state $\sigma_{\mathrm{DRx}}$ is the result of a balance between dislocation generation and strain hardening on one side and, on the other, dislocation annihilation and removal by GB migration (Fig. 37) [236,276,279-282]. The critical strain for DRX, $\varepsilon_{\mathrm{CD}}$ is always higher than $\varepsilon_{\mathrm{CS}}$ and increases with rising $\dot{\varepsilon}$ in the hot working domain whereas $\varepsilon_{\mathrm{CS}}$ decreases [230]. The subgrain dimensions $\lambda_{\mathrm{DRX}}$ in steady state are related to $Z$ and $\sigma_{\mathrm{DRX}}$ according to Eq. (15) and Eq. (16), $[216,267,278]$. Because of the limited strain $\left(\approx \varepsilon_{\mathrm{CD}}\right)$ before DRX, the substructure appears equiaxed and without microbands. The grains in steady state are approximately equiaxed and have a diameter $d_{\mathrm{DRX}}$ depending on $Z$ and $\sigma_{\mathrm{DRX}}$ in the following relation similar in type to Eq. (15)[17], [267,278,279]:

$d_{\mathrm{DRX}}=a^{\prime}+b^{\prime} \log Z$

$\sigma_{\mathrm{DRX}}=c^{\prime}+e^{\prime} d-d_{\mathrm{DRX}}^{-p^{\prime}}$

where $a^{\prime}, b^{\prime}, c^{\prime}, e^{\prime}$, and $p^{\prime} \approx 0.8$ are constants. 

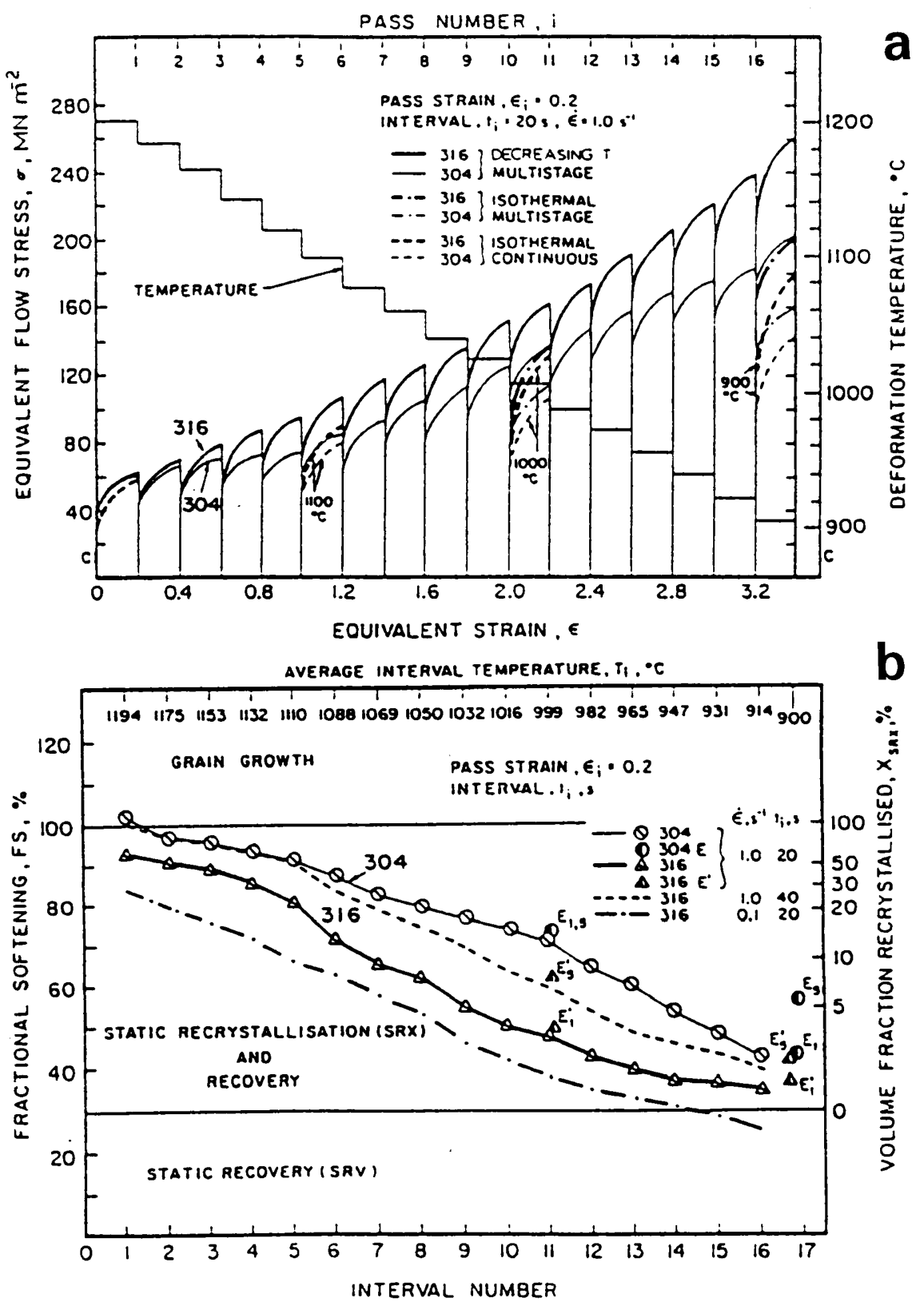

Fig. 36. Multistage simulation of a cross-country (parallel stands) bar mill at $\dot{\varepsilon}=1 \mathrm{~s}^{-1}$ for 304 and 316 stainless steels (a) the cumulative flow curves for equal passes $\varepsilon_{i}=0.2$, equal intervals $20 \mathrm{~s}$, and declining $T, 1200-900^{\circ} \mathrm{C}$ and (b) the degree of softening and SRX calculated from the drop in flow stress during each interval. The degree of SRX declines rapidly as $T$ falls and is lower for higher Mo contents. Microstructures, either optical or SEM-EBSI, confirm the absence of SRX in the last three intervals and appear similar to that produced by DRX at $900^{\circ} \mathrm{C}, 1 \mathrm{~s}^{-1}$ to the same total strain [263-266].

Since $d_{\mathrm{DRX}}$ is about ten times $\lambda_{\mathrm{DRX}}$, about one cell in $10^{3}$ becomes a nucleus usually at GBs or deformation bands $[216,244,275,279,281]$. Since $d_{\text {DRX }}$ also equals that of the first DRX grains in the necklaces that form along the initial grain boundaries, growth is considered to be limited by the formation of substructure in the new grains that eliminates the driving force. This re-establishment of substructure in the nuclei is an important cause of $\varepsilon_{\mathrm{CD}}$ being larger than $\varepsilon_{\mathrm{CS}}$ and for higher $\dot{\varepsilon}$
$[278,283]$. If held after straining, the steady state structure will undergo metadynamic recrystallization by continued growth of DRX nuclei reaching a size $d_{\mathrm{MRX}}$ larger than $d_{\text {DRX }}$ and following Eqs. (17) and (18), $[216,279,283]$.

DRX progress is thermally activated and its progress obeys the Avrami equations in similarity to SRX $[216,279]$. Thus, as the temperature declines the critical strain increases at a rising rate so that the possibility of 

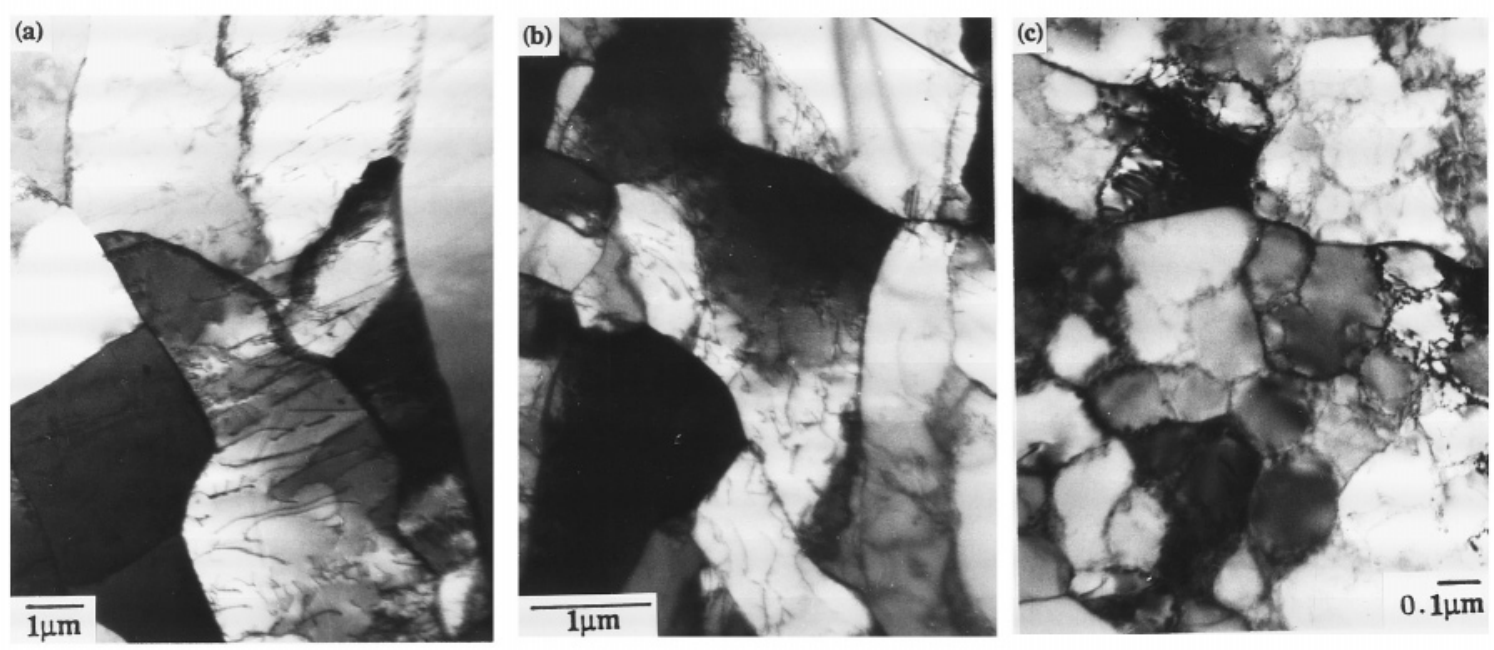

Fig. 37. Subgrain structures in 304 stainless steel during the steady state regime (a,b) or before the peak (c): (a) torsion $1200^{\circ} \mathrm{C}, 0.7 \mathrm{~s}-1$, (b) $1000^{\circ} \mathrm{C}$, and (c) tension $800^{\circ} \mathrm{C}, 0.001 \mathrm{~s}^{-1}$. The subgrain sizes have been shown to fit Eqs. (15) and (16) both before and after the peak, indicating that in DRX the substructure defines the strength [279].

DRX is cut off by fracture through GB cracking (whereas at the higher temperature DRX inhibits growth of cracking by isolating them from grain boundaries [216,276,279,284]. In addition, DRX is affected in the same way as SRX by alloying addition. Solute segregation to boundaries retards nucleation and may inhibit DRX even though SFE is decreased. Fine particles in sufficient density inhibit both nucleus formation and growth of the new grains [216,274,276]. Coarse particles may stimulate nucleation [279,280]; however, his may be possible only when solute reduces DRV which eliminates the high $\theta$ cells in hot working Al [218].

\subsection{Ferritic steels}

Ferritic steels (C, $\mathrm{Si}, \mathrm{Cr}$ stainless) undergo a high level of DRV with much similarity to $\mathrm{Al}$ alloys [190,217,233,237,284-286]; other body centered cubic alloys behave in the same way, notably $\beta$-Ti, $\beta-\mathrm{Zr}$, and Mo [286]. The literature surveyed seems to indicate that the solutes only slightly reduce DRV and do not give rise to alloy class behavior. In consequence of the high level of DRV, the ductility is an order of magnitude higher for $\mathrm{Fe}-25 \mathrm{Cr}$ (slightly less than $\alpha-\mathrm{Fe}$ ) than for $\mathrm{Fe}-25 \mathrm{Ni}$ (much less than $\gamma$-Fe with $\mathrm{C}$ ) even though it undergoes DRX $[217,233,286]$. While there is no equivalent to anodizing and POM for providing subgrain contrast as in $\mathrm{Al}$, the $\mathrm{SGB}$ in $\alpha \mathrm{Fe}$ do etch, more strongly as $\varepsilon$ rises. The GB become strongly serrated and thus indistinguishable from SGB; consequently a GRX-like mechanism occurs, although it has not been thoroughly investigated [190]. In distinction, at strains near 4, Lombry and Rossard [285] observed the formation of a network of boundaries with $\theta \approx 12^{\circ}$ enclosing regions of low $\theta$ subgrains and being distinct from the carbide decorated GB. These regions are smaller in scale than in deformation bands in Al, possibly because of the different slip symmetry in the body centered cubic crystals. The above behavior would lead one to conclude that the mechanisms described for $\mathrm{Al}$ are dominant and that reports of DRX stem from the same geometric subgrain serration developments. Classical DRX has been observed in zone refined $\alpha$-Fe in similarity to its discovery in 99.999 Al [196,287,288]. A comparison and contrast between ferrite and austenite have been drawn up [217,233]. Warm working of ferritic steels has the advantages of easier lubrication and diminished scaling; however, as the carbide content rises, the strength rises and ductility decreases to above and below those for austenitic steels [233,286,289].

\subsection{Warm working}

Reduction in temperature and increase in strain rate (increase in $Z$ ) reduce the level of DRV that is achieved. As the stress rises all the average microstructural spacings are decreased which means the extremes of wall densities are much higher with the consequent increase in misorientation $[228,236,290]$. At the upper end of the warm working range, $0.4-0.5 T_{\mathrm{m}}$, the substructures remain equiaxed and the SGB appear to be ephemeral, thus the walls of higher $\theta$ likely do not persist. At the lower end, $0.3-0.4 T_{\mathrm{m}}$, the subgrains become somewhat elongated with very ragged walls more of which have large $\theta[223,291]$. Moreover, such walls become more persistent; however, the evidence is not readily available since the ductility in torsion is limited. 
While $Z$ adequately defines the condition in the core of the hot working range, it does not at the borders. Decreasing $T$ ( $\dot{\varepsilon}$ const) reduces the mobility of the GB so that, while reduced DRV increases the dislocation density, creation and growth of the nuclei become very slow. Increasing $\dot{\varepsilon}$ does not directly affect GB mobility but increased driving force does speed up DRX $\left(\varepsilon_{\mathrm{c}} / \dot{\varepsilon}\right.$ decreases) until high $\dot{\varepsilon}$ straining develops substructure in the nuclei so quickly that formation is retarded and finally inhibited $\left(\varepsilon_{\mathrm{c}}\right.$ increases). As a consequence of these dependencies, in the warm working domain discontinuous DRX does not take place [230].

In the warm working regime, the concentration of high misorientation subboundaries increases as DRV is reduced; however, it cannot be maintained that this is DRX any more than cold worked material is considered recrystallized because it contains regions of high misorientation $[2,35,236]$. It is normally accepted that the cold deformation creates all of the high misorientation features, which lead to nucleation and recrystallization textures during annealing [2,35]. In addition, it is not logical to classify the deformed partially recovered substructure with some high misorientation boundaries as so called continuous dynamic recrystallization $^{1}$ (cDRX) [2,292] since this would also apply to the remaining cold worked regions in partially discontinuously recrystallized material [236]. It is currently accepted that some particle-bearing alloys, such as $\mathrm{Al}-$ $\mathrm{Cu}-\mathrm{Zr}, \mathrm{Al}-10 \mathrm{Mg}-\mathrm{Zr}$, or $\mathrm{Al}-\mathrm{Li}-\mathrm{Cu}-\mathrm{Mg}-\mathrm{Zr}$, subjected to a prior low temperature, high strain thermo mechanical process, convert to a fine grained microstructure by cDRX during the initial stages of superplastic straining [169,194,225,234,236,293]. Because deformation of stabilized alloys at a higher $T$ and lower $\dot{\varepsilon}$ cannot enhance DRV causing repolygonization to the less dense characteristic substructure, sliding and rotation proceeds on the high angle boundaries which have undergone enhanced conversion to GB due to the shear stress [169].

In industrial hot working, the departure from the ideal state towards warm working may be quite great due to inhomogeneous deformation, both $\varepsilon$ and $\dot{\varepsilon}$, related to shape and friction of the tooling and nonuniform cooling from cold tooling. In addition, objects in multistage forming processes associated with a single preheating undergo a gradual cooling during the sched-

\footnotetext{
${ }^{1}$ This terminology is widely used but does not meet the definition of recrystallization in this review (see Section 1). Continuous static recrystallization, sometimes called 'in situ', is a mechanism found primarily in heavily deformed alloys with substructure strongly stabilized by fine particles so that during prolonged annealing, nucleation and growth of new grains (discontinuous RX) is severely retarded. Hornbogen and other $[2,169,194,218,225,229,292,293]$ have observed that many high misorientation walls become relaxed boundaries but, though mobile, do not migrate. The final grain size is about the same as that of cells or subgrains.
}

ule; in fact, the temperature near the surface falls quite rapidly from contact with the tooling and rises again due to deformation heating near the center. Even laboratory rolling is subject to the above constraints which may be acerbated by the limited initial thickness and the very high strains employed to avoid intermediate arrests with the possibility of SRX [258]. In many multistage schedules, the formation of new fine grains during the few initial intervals reduce texture formation but do gradually eliminate the initial large, irregular segregated as-cast structure. Given all of these effects, it is not surprising that the processed metal contains a 'warm worked' substructure with possibly high misorientation subgrain facets [265].

\section{The role of grain boundaries in recrystallization}

\subsection{Introduction}

Consider the case of a deformed polycrystalline metal subjected to a temperature sufficient to initiate recrystallization. Further, consider the microstructure at a time after new recrystallized grains have nucleated. It is well known that recrystallization will proceed by the migration of the new grain boundaries into the deformed matrix leaving behind what are commonly known as 'strain-free grains'. The migration is driven by the energy gradient across the boundary due to the presence of the deformed structure on one side and the recrystallized structure on the other. The rate at which the boundaries advance depends on the misorientation of the lattices on each side of the boundary, the plane of the boundary relative to the lattices, the chemistry of the boundary, the deformation gradient across the boundary, pressure and temperature.

In this section, we examine each of these issues. For brevity, we make liberal use of references from a rich literature. Several comprehensive reviews of grainboundary migration exist. A good starting place is the text by Gleiter and Chalmers [294]. More recently, the subject has been covered in great detail by Humphreys and Hatherley [2]. The book of Sutton and Balluffi is an excellent general reference to interface science [295].

\subsection{Background}

\subsubsection{Grain boundaries in metals}

A grain boundary is formed where two single-crystal grains in a polycrystalline aggregate meet. The boundary is characterized by its macroscopic and microscopic degrees of freedom [296]. In its ideal form, the boundary is planar and defined by the misorientation of the grains on either side of the boundary (two degrees of freedom for the axis of misorientation and one for the misorientation angle) and the plane of the 
interface (two degrees of freedom). The rigid-body shifts, parallel and perpendicular to the boundary plane, comprise the three microscopic degrees of freedom. The general grain boundary is not planar and can take on curvatures consistent with the energetics of the system.

It is common practice to describe grain boundaries by the misorientation of one grain with respect to another. It is convenient to use the axis-angle notation to denote the rotation axis and the rotation angle necessary to transform one into the other. Consider two crystal lattices misoriented with respect to each other and allowed to interpenetrate. At certain axis-angle pairs, the lattices form special patterns characterized by the coincident site lattice (CSL) notation, e.g. see [297]. In this notation, the misorientation is denoted as $\Sigma n$ where $n$ is the reciprocal density of coincident lattice sites. $n$ is always odd and for symmetric tilt grain boundaries (boundaries where the boundary plane contains the rotation axis and bisects the rotation angle) can be calculated by knowing the plane of the boundary $(h k l)$ :

$n=h^{2}+k^{2}+1^{2}$ for $h^{2}+k^{2}+1^{2}$ odd

$n=\frac{h^{2}+k^{2}+1^{2}}{2}$ for $h^{2}=k^{2}+1^{2} \quad$ even.

For asymmetric tilt grain boundaries, $\mathrm{n}$ can be calculated from [298]

$n=\frac{h_{1}^{2}+k_{1}^{2}+1_{1}^{2}}{h_{2}^{2}+k_{2}^{2}+1_{2}^{2}}$

where subscripts 1 and 2 refer to grains on either side of the boundary.

It should be emphasized that the CSL notation is geometrical only and disregards the plane of the grain boundary and the microscopic degrees of freedom. Consequently, we might not expect that macroscopic properties would necessarily correlate with $\Sigma n$. However, there is some evidence that such a correlation exists for some properties, discussed below.

Grain boundaries are often grouped into broad classes, such as, low- and high-angle, twist and tilt, and special and random. The first class is based on structure and energy criteria while the second and third classes are strictly geometrical in nature. Conventionally, the delimiting angle separating low from high angle boundaries is $15^{\circ}$; for cubic crystals [299]. This is approximately the angle where it is not longer possible to discern well separated dislocations forming the boundary [300].

Strictly speaking, special boundaries (boundaries which have low $\Sigma$ and exhibit special properties) occur at well defined angles [301], but it has been shown that boundaries near an exact $\Sigma$ misorientation can exhibit
$\Sigma$-like properties. The acceptance angle, $\Delta \phi$, over which boundaries exhibit $\Sigma$-like properties is usually expressed as

$\Delta \phi=\Delta \phi_{0} \Sigma^{-m}$

Table 1 give a list of the criteria that have been proposed [299,302-304]. The Brandon criterion [299] is the most commonly used.

Not all boundaries that meet these criteria exhibit special properties. Generally speaking, special boundaries are those boundaries with $\Sigma \leq 20$ [305]. Other boundaries, including $\Sigma>29$, are considered random. This separation of special from random at $\Sigma 29$ has been deduced from experience of correlating special fractions (total number of boundaries $1 \leq \Sigma \leq 29$ /total number of boundaries) with observed properties [305]. The distribution of boundary types with respect to $\Sigma$ is called the grain boundary character distribution. It may be that the $\Sigma$ threshold, above which the grain boundaries lose their special properties, depends on the property under investigation and the external conditions, i.e. pressure and, especially, temperature $[306,307]$. The correlation of properties with $\Sigma$ is troubling at first since for example, a $\Sigma 3$ coherent twin always has special properties whereas the $\Sigma 3$ incoherent twin may not. The difference between these two cases is, of course, the plane of the grain boundary. Randle has recently reviewed the available literature regarding the role of grain boundary plane in cubic polycrystal and has found a correlation of segregation, precipitation, energy, and cracking with grain boundary plane (V. Randle, personal communication, 1997). That work indicates that characterization of misorientation is necessary but not sufficient for a detailed correlation with properties.

It has been well established in the literature that grain boundary segregation depends on grain boundary structure [308-313]. More specifically, the experimental evidence is that special boundaries tend to have less segregation of impurities than random boundaries. For the case NiIn, it was observed that the enthalpy of segregation at near- $\Sigma 19$ boundaries was $\sim 73 \%$ of the enthalpy of segregation measured in polycrystal $[309,312]$.

Table 1

Criteria for the acceptance angle, $\Delta \phi$, over which boundaries exhibit $\Sigma$-like properties

\begin{tabular}{lll}
\hline$\Delta \phi_{0}$ & $\mathrm{~m}$ & References \\
\hline 15 & $-1 / 2$ & {$[299]$} \\
- & $-2 / 3$ & {$[302]$} \\
- & -1 & {$[303]$} \\
- & $-5 / 6$ & {$[304]$} \\
\hline
\end{tabular}


Table 2

Table of driving forces from [294]

\begin{tabular}{|c|c|c|c|}
\hline Source & Equation for the driving force & Estimated driving force $\left(\mathrm{N} \mathrm{m}^{-2}\right)$ & Assumed experimental constants \\
\hline Grain-boundary energy & $P=\sigma / a$ & $2 \times 10^{4}$ & $\begin{array}{l}\sigma=10^{-2} \mathrm{~J} \mathrm{~m}^{-2} \\
a=10^{-4} \mathrm{~m}\end{array}$ \\
\hline Surface energy & $P=\frac{2 \Delta \sigma^{\prime}}{d}$ & $2+10^{4}$ & $\begin{array}{l}d=10^{-5} \mathrm{~m} \\
\Delta \sigma^{\prime}=10^{-3} \mathrm{~J} \mathrm{~m}^{-1}\end{array}$ \\
\hline Magnetic field & $P=\frac{\Delta k}{2} H^{2}\left(\cos ^{2} \theta_{1}-\cos ^{2} \theta_{2}\right)$ & $10^{3}$ & $\begin{array}{l}H=10 \mathrm{~T} \\
\theta_{1}=36^{\circ} \\
\theta_{2}=72^{\circ} \\
\text { Material: } \mathrm{Sb}\end{array}$ \\
\hline Strain field & $P \approx \frac{s^{2}}{2}\left(\frac{1}{E_{1}}-\frac{1}{E_{2}}\right)$ & $2.5 \times 10^{2}$ & $\begin{array}{l}s=10^{7} \mathrm{~J} \mathrm{~m}^{-2} \\
\left(\frac{1}{E_{1}}-\frac{1}{E_{2}}\right)=5 \times 10^{-12} \mathrm{~m}^{2} \mathrm{~N}^{-1}\end{array}$ \\
\hline Temperature gradient & $P=\frac{\Delta S \cdot 2 \delta \operatorname{grad} T}{V^{*}}$ & $4 \times 10^{1}$ & $\begin{array}{l}\Delta S=2 \text { cal g-atom } \\
\text { grad } T=10^{5} \mathrm{~K} \mathrm{~m}^{-1} \\
2 \delta=5 \times 10^{-10} \mathrm{~m} \\
V^{*}=10 \mathrm{~cm}^{3} \text { g-atom }^{-1}\end{array}$ \\
\hline
\end{tabular}

\subsubsection{Recrystallization}

The mobility, $m$, of a grain boundary is [314]

$m=\frac{v}{p}=\frac{v}{\rho G \boldsymbol{b}^{2}}$,

where $v$ is the velocity of the advancing grain boundary, $p$ is the driving force, $\rho$ is the dislocation density, $G$ is the shear modulus, and $\boldsymbol{b}$ is the Burger's vector. Table 2 shows estimates for the magnitude of the driving force in Eq. (22) for various methods of imposing the driving force [294]. In this discussion, we are most interested in driving forces due to stored energy from deformation (for recrystallization) and due to grainboundary energy (for controlled experiments).

Recrystallization is a thermally activated process exhibiting Arrhenius behavior. It has proved difficult to make systematic studies of boundary migration under the constant driving force of stored energy of deformation. However, if there is no change in mechanism when the driving force changes, then it is possible to make careful measurements of migration velocity using a special sample configuration where the driving force is the interfacial energy to determine the prefactor and activation enthalpy in the Arrhenius relation. These results can then be applied directly at higher driving forces through Eq. (22).

Dunn [294,315] suggested the geometry of the specimen shown in Fig. 38 to probe the effect of misorientation on mobility. The driving force is the ratio of the grain boundary energy to the radius of the shrinking grain, $\sigma / a$. For this experiment, it is common to write the Arrhenius expression in terms of the reduced mobility $m \sigma$,

$$
A=v a=m \sigma=A_{0} \exp \left(-\frac{H^{\mathrm{M}}}{k T}\right)
$$

where $H^{\mathrm{M}}$ is the activation enthalpy of migration and $A_{0}$ is the prefactor. Table 3 shows how $A_{0}$ and $H^{\mathrm{M}}$ are influenced by the experimental variables. The geometry in Fig. 38 is produced by careful cutting and annealing of a bicrystal. When the specimen is annealed, Grain I grows and Grain II shrinks by migration of the curved section of the boundary. Under these conditions, the boundary radius, $a$, is constant so the driving force remains constant during the experiment. This geometry has been successfully applied by a number of authors [316-326]. Using novel experimental techniques such as those employed in Aachen [314,318,320,327,328], it is possible to make valid determinations of $A_{0}$ and $H^{\mathrm{M}}$ as a function of the parameters in Table 3 .

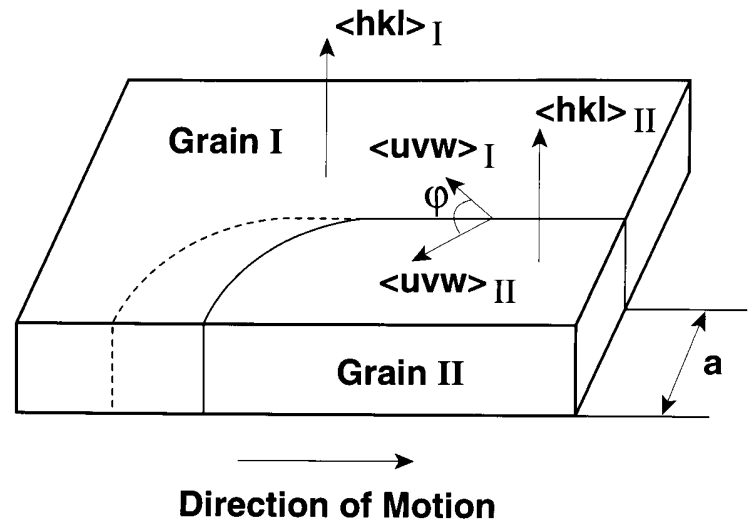

Fig. 38. Bicrystal geometry used for grain boundary migration measurements under a constant driving force. 
Table 3

Schematic illustration of the influence of misorientation, boundary plane, chemistry, deformation, and pressure on the activation enthalpy and prefactor for grain boundary migration

\begin{tabular}{llllll}
\hline & Misorientation & Boundary plane & Chemistry & Deformation & Pressure \\
\hline$A_{0}$ & $\bullet$ & $\ominus$ & & $\bullet$ \\
$H^{\mathrm{M}}$ & $\bullet$ & $\ominus$ & $\bullet$ & $\ominus$ \\
\hline
\end{tabular}

๑, strong influence; $\ominus$, some influence.

\subsection{Which boundaries play a role in recrystallization?}

Grain boundaries can be characterized by their relative twist and tilt components. Little work has been done to characterize the relative fraction of twist and tilt boundaries in a microstructure due to the difficulty of conducting experiments to determine both misorientation and boundary plane, but this has been accomplished in a few cases, e.g. the work of Randle in fcc metals (Fig. 39) showed that there are many more tilt boundaries in fcc polycrystal than twist boundaries [329]. This is consistent with what one might expect from theoretical calculations, e.g. those of Merkle and Wolf [296] shown in Fig. 40. On the basis of their relatively low fractions, we might expect that twist boundaries play a rather minor role in recrystallization. Experiments in recrystallizing single-crystal aluminum (Table 4) also revealed tilt-type boundaries (although the statistics are quite low) [330,331].

Randle has recently found that (V. Randle, personal communication, 1997):

1. Asymmetric tilt grain boundaries predominate in some fcc metals

2. Symmetric tile grain boundaries predominate in some other metals, e.g. $\mathrm{Ni}_{3} \mathrm{Al}, \mathrm{Si}$ and $\mathrm{Nb}$

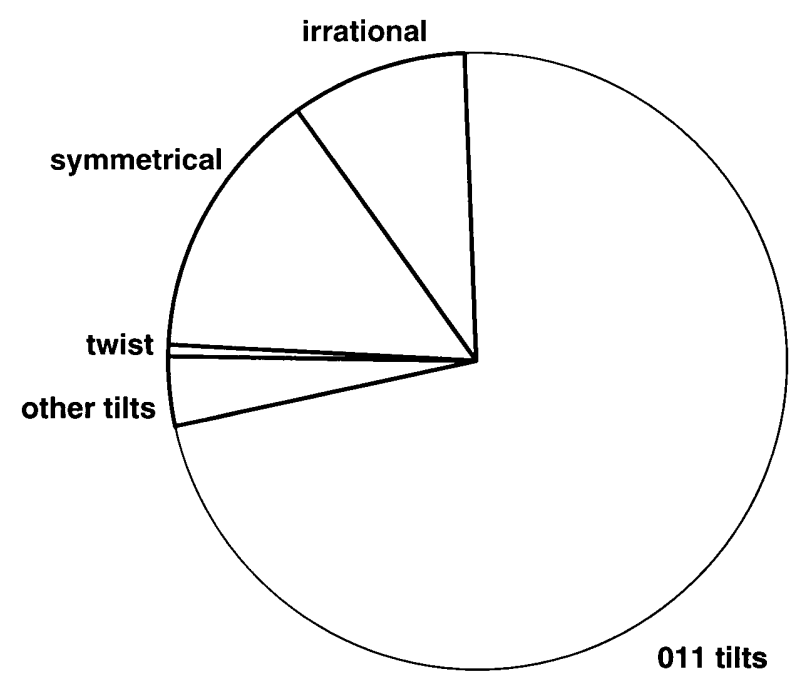

Fig. 39. Distribution of grain boundary plane types an annealed $\mathrm{Cu}$ [329].
The relative fraction of special versus random and low angle versus high angle boundaries depend on processing. As discussed below, it is well known that low angle boundaries migrate slower than high angle boundaries. Therefore, generally speaking, the boundaries dominating the recrystallization process are expected to be high angle, tilt-type boundaries.

\subsection{Grain boundary structure-chemistry linkage}

Table 3 shows that the migration enthalpy is strongly affected by both structure and chemistry. The effect is so closely coupled that the two issues must be discussed together. The first indications of this coupling came in the classic work of Rutter and Aust [332-334] shown in Fig. 41(a) and (b). In this work, at least three important observations were made:

1. Low angle boundaries migrate slower than high angle boundaries.

2. Special boundaries migrate faster than random boundaries.

3. Segregation generally reduces the migration rate of all boundaries.

Support for these conclusions have been reported by others [2,314,318-320,322,323,325,328,335,336]. Perhaps the most comprehensive set of data have been measured for Al (Fig. 42(a)-(c)) [322,337-341]. In those works, activation enthalpies and prefactors have been determined under constant driving force conditions for $\langle 100\rangle,\langle 110\rangle$, and $\langle 111\rangle$ symmetric tilt grain boundaries.

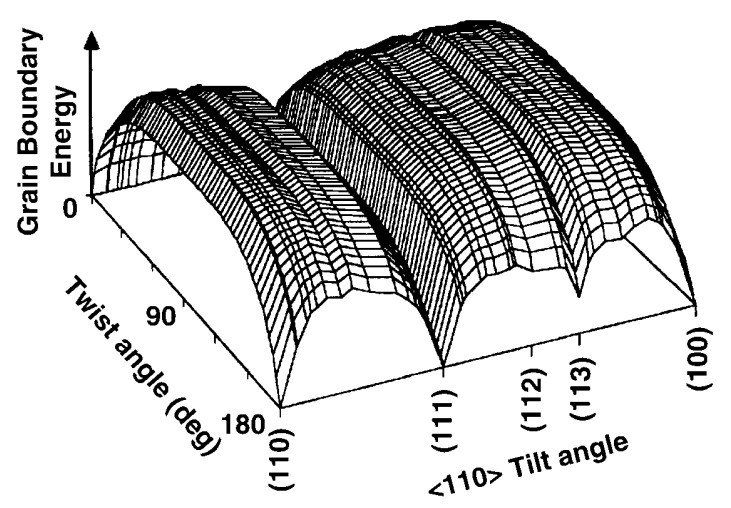

Fig. 40. Energy vs. twist and tilt angle calculated using a LennardJones potential for $\mathrm{Cu}[296]$. 
Table 4

Boundary type, velocity, and activation energy for boundaries studied in Al [330,331]

\begin{tabular}{|c|c|c|c|c|c|c|}
\hline \multirow[t]{2}{*}{ Specimens } & \multirow[t]{2}{*}{ Character } & \multirow[t]{2}{*}{$\phi(h k l)$} & \multicolumn{3}{|c|}{$v\left(10^{-5} \mathrm{~cm} \mathrm{~s}^{-1}\right)$} & \multirow[t]{2}{*}{$H^{\mathrm{M}}\left(\mathrm{kJ} \mathrm{mol}^{-1}\right)$} \\
\hline & & & $801 \mathrm{~K}$ & $833 \mathrm{~K}$ & $851 \mathrm{~K}$ & \\
\hline 1 & Mixed & $(100)$ & - & 3.9 & 13 & 419 \\
\hline 1 & Tilt & $(01 \overline{1})$ & - & 5.5 & 16 & 360 \\
\hline 2 & Mixed & $(0\{1$ bar $\} 0)$ & 0.5 & 7.6 & 27 & 440 \\
\hline
\end{tabular}

Segregants interact with the boundary exerting a 'solute drag' effect generally raising the migration enthalpy. The segregant's effect can be divided into five regimes: (1) concentrated alloys, (2) low purity, (3) high purity, (4) ultrahigh purity, and (5) completely pure $[342,343]$. These regimes are illustrated schematically in Fig. 43(a) and are to be compared with the experimental results in Fig. 43(b) [342] and Fig. 43(c) [343]. These figures illustrate how the activation enthalpy for boundary migration varies with misorientation for tilttype grain boundaries for various levels of purity. Let us first take the case of high purity materials, case (3). The highest activation enthalpy is at the lowest angle of misorientation. The activation enthalpy decreases and then oscillates with minima at the special misorientations. This is the range of purity where the special and random boundaries exhibit different segregation behaviors. When the material is of ultrahigh purity, case (4), special and random boundaries migrate with the same activation enthalpy. It is believed that if the material had no impurities, case (5), special boundaries would have a higher migration enthalpy than random boundaries due to their lower energy [342]. At higher impurity levels, case (2), both special and random boundaries are thought to saturate with impurities, again as in the ultrahigh purity case, washing out the anisotropy of migration.

Concentrated alloy, case (1), are known to exhibit anisotropy in their migration enthalpies [326,343-345]. The migration behavior is similar to the case of completely pure materials with special boundaries migrating slower than random boundaries. Lejcek and Adamek have proposed to explain this observation throughout the migration enthalpy [343]. The migration enthalpy can be separated into three contributions: intrinsic, segregation, and mixing.

$H^{\mathrm{M}}=H_{\mathrm{int}}^{\mathrm{M}}+H_{\mathrm{seg}}^{\mathrm{M}}+H_{\text {mix }}^{\mathrm{M}}$

The segregation and mixing terms can be expressed as

$H_{\mathrm{seg}}^{\mathrm{M}}=\left(X-X^{\phi}\right) H_{\mathrm{seg}}$

and

$H_{\mathrm{mix}}^{\mathrm{M}}=X(1-X) W_{\mathrm{AB}}$ where $X$ is the bulk atomic concentration of the solute, $X^{\phi}$ is the grain boundary concentration, $H_{\text {seg }}$ is the segregation enthalpy of the solute in the matrix, and $W_{\mathrm{AB}}$ reflects differences in bonding energies, $\varepsilon_{i j}$, between different nearest neighbour atoms in the matrix, $W_{\mathrm{AB}}=2 \varepsilon_{\mathrm{AB}}-\varepsilon_{\mathrm{AA}}-\varepsilon_{\mathrm{BB}}$. It should be pointed out that there is at least one example of impurities that accelerate the migration rate of grain boundaries: the case of $\mathrm{Ga}$ in $\mathrm{Al}$ [321].

\subsection{The effect of pressure}

Several authors have studied the effect of hydrostatic pressure, $P$, on grain boundary mobility $[319,323,325,335,346,347]$. High hydrostatic pressure can be used to probe the activation volume for grain boundary migration. By definition, the activation volume is the volume difference between the activated state and the ground state [314]. The activation enthalpy can be expressed as the sum of the activation energy, $E$, and $P V^{*}$, where $V^{*}$ is the activation volume,

$$
\begin{aligned}
& A=A_{0} \exp \left(-\frac{H^{\mathrm{M}}}{k T}\right)=A_{0} \exp \left(-\frac{E+P V^{*}}{k T}\right), \\
& V^{*}=-\left.k T \frac{\partial \ln A}{\partial P}\right|_{T}
\end{aligned}
$$

$V^{*}$ can be determined from measurements of $A$ as a function of hydrostatic pressure. Results have shown that for aluminum, $\langle 110\rangle$ tilt boundaries appear to move by some cooperative motion of several atoms while $\langle 100\rangle$ and $\langle 111\rangle$ tilt boundaries may move by a single atom mechanism. However, these results are not definitive [314]. An interesting observation is that at elevated pressures (up to $1.5 \mathrm{GPa}$ ) low angle boundary migration seems to become frozen, while the diffusionless processes of general boundary migration are not affected [347].

The activation volume can also be determined calculationally [296] or experimentally through structural studies [348,349]. Fig. 44 shows the calculated dependence of the grain boundary energy on the activation volume. Up to this time there have been no successful determinations of the activation volume from structural studies. This is due to experimental complications asso- 
ciated with abberations in the high resolution electron microscope that have just recently been identified [350].

\section{Recommendations for further research}

(1) A better experimental understanding is needed of the details of the deformed state, both from experimental studies and from developments of the understanding of deformation theory. The needed insights include knowledge and prediction of the length scale of homogeneous deformation within grains under a range of starting microstructures (initial grain size, particle den-
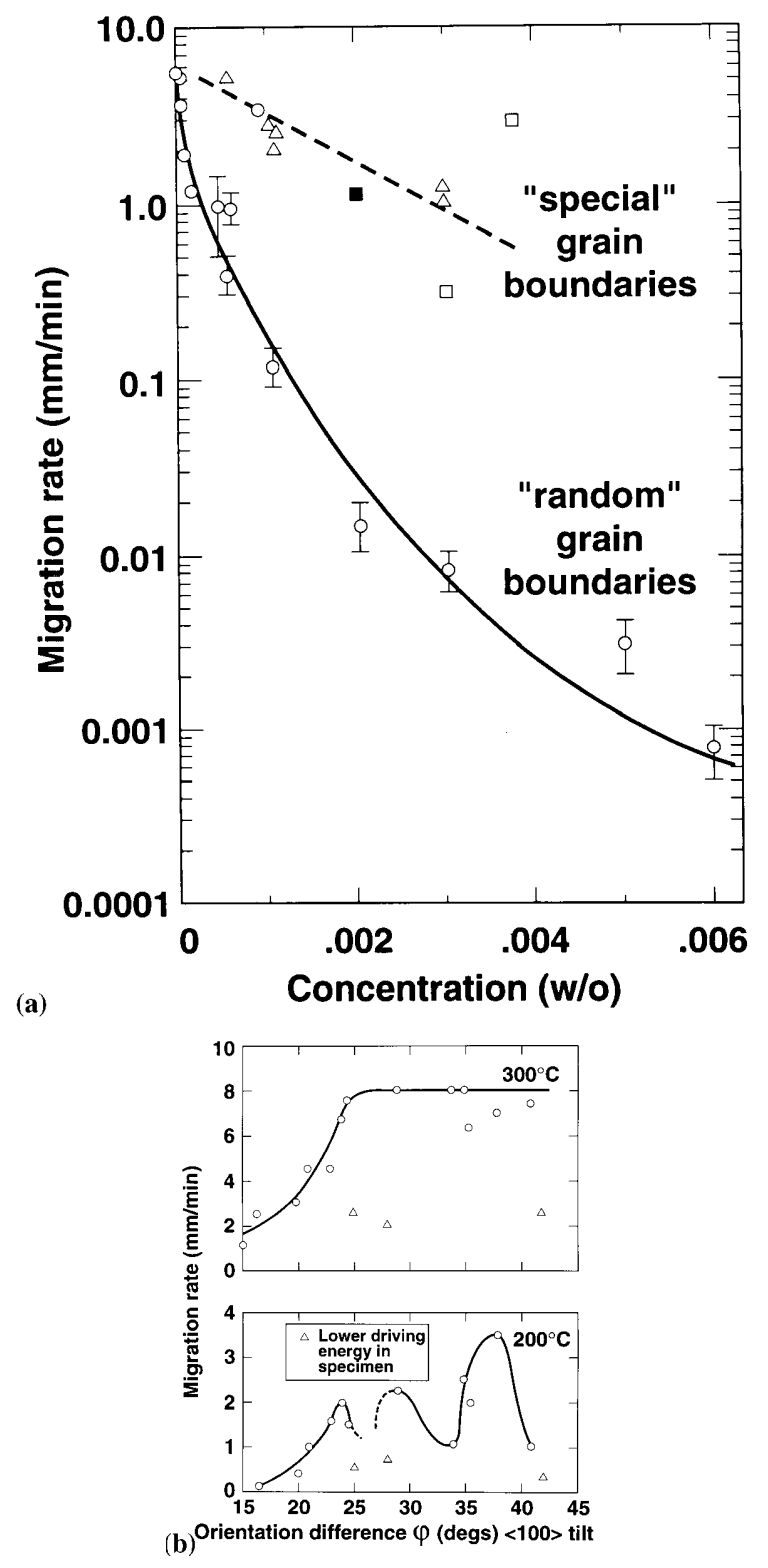

Fig. 41. (a) Boundary migration rate studied at $300^{\circ} \mathrm{C}$ in $\mathrm{Pb}-\mathrm{Sn}$ alloys as a function of $\mathrm{Sn}$ concentration [332-334]. (b) Rate of boundary migration at $300^{\circ} \mathrm{C}$ (top) and $200^{\circ} \mathrm{C}$ (bottom) as a function of misorientation for $\langle 100\rangle$ tilt boundaries in $\mathrm{Pb}$ [332].
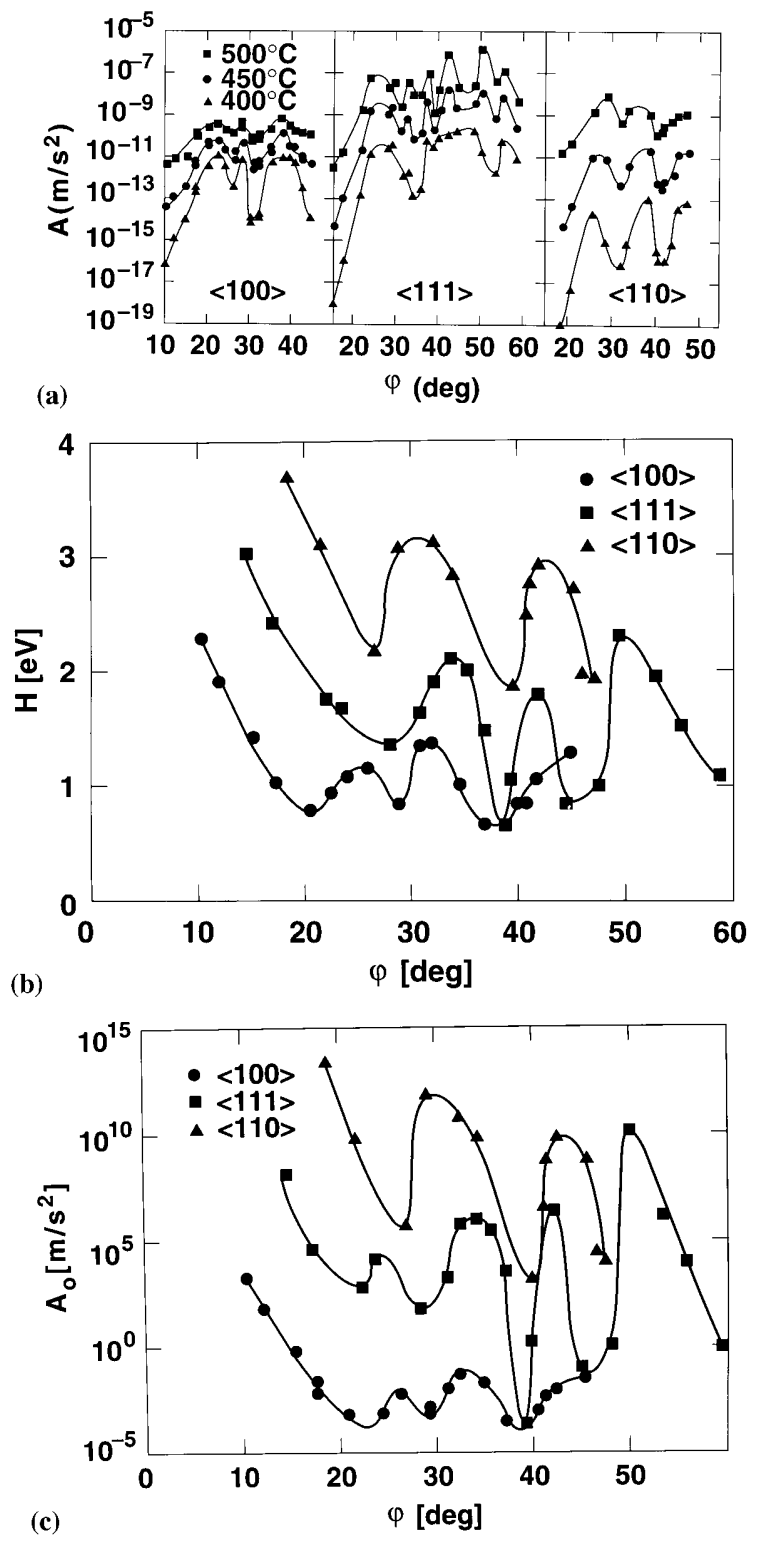

Fig. 42. (a) Misorientation dependence of the tilt grain boundary mobility in $\mathrm{Al}$ for misorientation axes $\langle 100\rangle,\langle 110\rangle$ and $\langle 111\rangle$ [337]. (b) Activation energy as a function of misorientation in $\mathrm{Al}$ for misorientation axes $\langle 100\rangle, \quad\langle 110\rangle$ and $\langle 111\rangle$ [322,338,339,337,340,341]. (c) Prefactors as a function of misorientation in $\mathrm{Al}$ for misorientation axes $\langle 100\rangle,\langle 110\rangle$ and $\langle 111\rangle$ $[322,338,339,337,340,341]$.

sities, solute contents) and deformation conditions. The boundaries between he homogeneously deforming regions are misoriented 'transition bands' [14] whose magnitude of misorientation and the distance over which it occurs needs to be known. Also, the orientation dependence of stored energy and the details of strain localization (shear banding) and its effect on microstructure are critically needed for a wide range of materials and deformation conditions.

(2) A new experiment will be useful that determines the dislocation rearrangement in recovery and the vari- 
ous processes of subgrain growth, including both the general processes that lower the average stored energy and special processes such as subgrain coalescence that can and does lead to local stored energy heterogeneities.

(3) Not discussed in this paper for lace of space, but for clear scientific and, in particular, industrial interest,

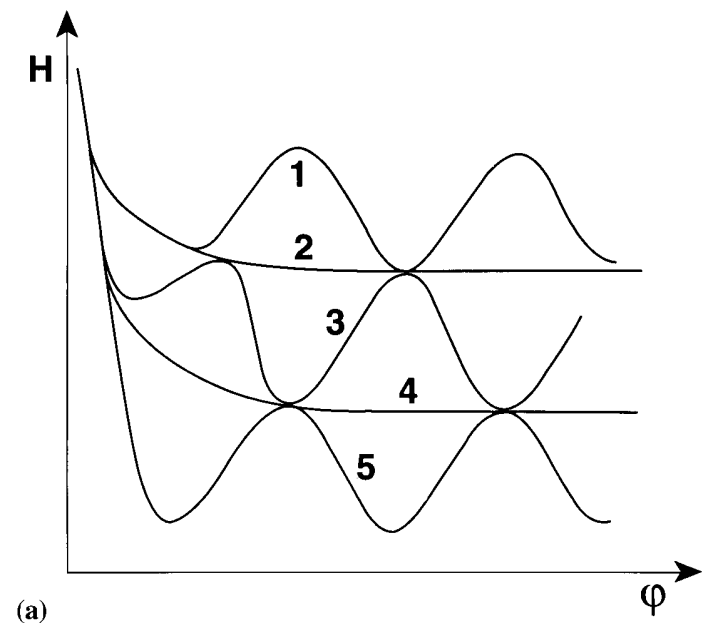

(a)

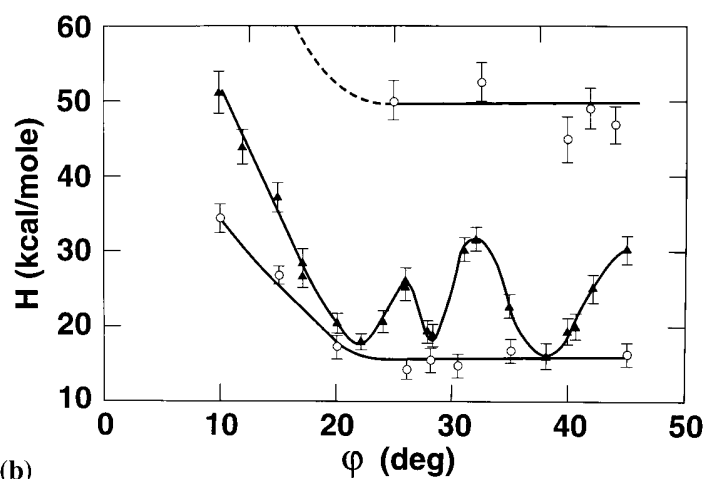

(b)

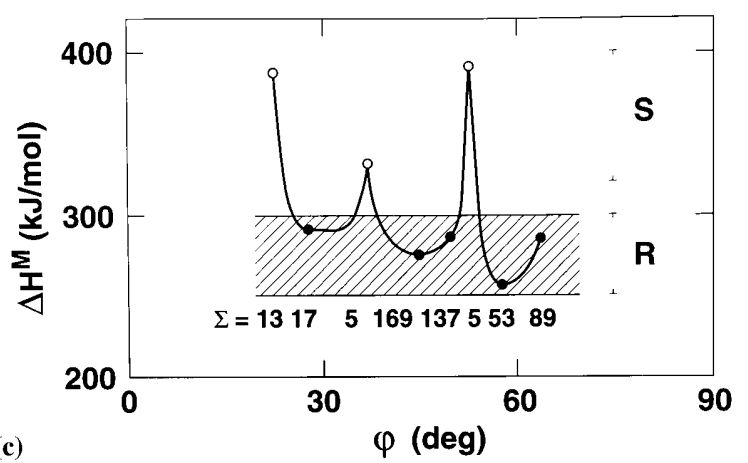

Fig. 43. (a) Schematic dependence of activation energy for grain boundary mobility for differently purity levels: (1) concentrated alloy, (2) low purity, (3) high purity, (4) ultra-high purity, and (5) completely pure. (b) Measured dependence of activation energy for grain boundary mobility for $\langle 100\rangle$ tilt boundaries in $\mathrm{Al}$ of different purity levels: $\square 99.9995$ at.\%, $\triangle 99.9992$ at.\%, $\bigcirc 99.98$ at.\% [342]. (c) Measured dependence of activation energy for grain boundary mobility for $\langle 100\rangle$ tilt boundaries in $\mathrm{Fe}-6$ at.\% $\mathrm{Si}$ at different purity levels [343]. S and R refer to special and random grain boundaries, respectively.

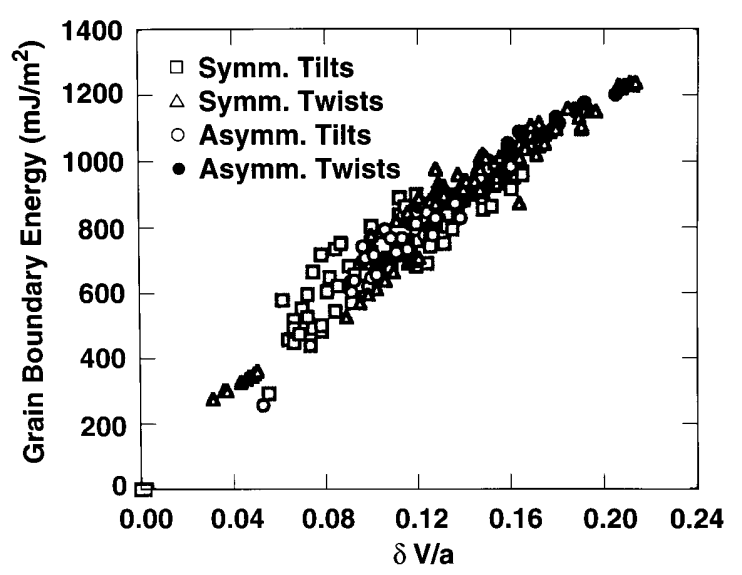

Fig. 44. Correlation between grain boundary energy and grain boundary free volume as determined from simulation [296].

is how the deformation and annealing processes in high solute alloys such as those used for precipitation hardening are affected by the deformation and annealing of unstable alloys in which precipitation can and occur during cooling and where the precipitates then dissolve on solution heating that can lead to different amounts of recrystallization.

(4) Development of deformation models for grain subdivision, misorientation angle development and stored energy distributions.

(5) Development of new recrystallization models which take the subdivision of the deformed matrix material into account.

(6) In-situ investigations of growth of a single nucleus/grain in the bulk of a large polycrystalline sample by high energy synchrotron radiation diffraction.

(7) The general trends and qualitative understanding in this subject need to be made as quantitative as possible for use in modelling. As discussed in the conference [351], recrystallization modelling, like all microstructural modelling, serves at least two valuable purposes. First is the ability to test if the current models can fit experimental data in a satisfactory way using physically reasonable fitting parameters and, second, after the first has been achieved, to use the models to predict experimental behavior not yet measured. More specifically, extension of 2D recrystallization models to three dimensions is important as will including a direct description of interfaces to recrystallization modelling, and the inclusion of experimental data into models (e.g. microstructure, properties, etc.).

(8) Investigation of early stages of recrystallization in bcc systems to determine why certain texture components nucleate at higher rates than others.

(9) Many aspects of recrystallization in particle-containing alloys are understood reasonably well qualitatively. However, in many cases the theories are not 
sufficiently soundly based, and the experimental database is small. In order to account quantitatively for the recrystallization behavior, further work is needed in several area, in particular:

- Measurements of size, shape, and orientation distribution at particle deformation zones and bands (PDZr, PDBr) as a function of strain, particle size, and grain orientations.

- Modelling the heterogeneous deformation of large particles.

- Experimental determination of the temperature, strain rate, and particle size conditions under which PDZs are formed, from a wider range of materials than at present.

- Basic experiments on the conditions of PSN, effects of strain, particle size, and shape.

- Determination of PSN nucleation rates and sites, e.g., how good an approximations the saturation?, are particles at boundaries and other heterogeneities more effective at PSN sites?, and Why is the PSN efficiency typically $\doteq 19$ ?

- Quantitative experiment and theory to determine the effects of small particles on slip distributions (e.g. shear band formations) and on the cell/subgrain size and misorientation and dislocation density.

- Basic experimental work on the site strain between precipitation and annealing.

- Determination of the magnitude of Zener pinning during recrystallization and any orientation.

(10) With regard to oriented nucleation, efforts must be directed at assessing the relative importance in various alloy systems of (i) low stored energy, (ii) particle stimulated, (iii) twin-related, and (iv) shear band nucleation. With respect to selective growth phenomena, the relative mobilities of different classes of grain boundaries should be determined; also of importance is the question of variant selection and the extent to which it operates at elevated temperatures in various alloy systems.

(11) The role of twinning in the nucleation and propagation of DRX should be clarified, particularly in low SFE polycrystalline materials.

(12) Generalized DRX models are required that can predict texture development during deformation along arbitrary strain paths. These should be based on more detailed knowledge regarding the physical characteristics of nucleation and growth, as outlined above.

(13) Outstanding issues regarding the kinetics of metadynamic recrystallization should be resolved, with particular emphasis on how these differ from those that apply to static recrystallization.

(14) More information is needed regarding the interaction between precipitation and DRx in rolling mills. The influence of accumulated time versus interpass time should be clarified, as should the effects of precipitate coarsening during deformation.
(15) More needs to be known about the effects of the vacancies generated during high temperature deformation on both DRX and MDRX. The accelerated diffusion attributable to the presence of the vacancies may help to resolve outstanding issues regarding the kinetics of these two mechanisms.

(16) In particular, the early stages of the continuous reaction, and the role of solutes and precipitates, need much more study.

(17) The generality of GDX in especially high stacking fault energy metals and alloys needs to be confirmed.

(18) The hot deformed steady state substructures need to be better characterized in terms of subgrain size, misorientation distributions and texture by measuring every subgrain orientation. This needs to be done as a function of temperature and strain rate as well as strain during the transient. The same type of study needs to be made in the warm working domain to see how cold working transforms into hot working, notably for high stacking fault energy metals.

(19) Important outstanding issues include the mechanism by which the energy gradient across the grain boundary (in the form of dislocations) causes the grain boundary to advance (by way of the adsorption of the dislocations at the interface).

(20) The interaction of structure, chemistry and mobility of boundaries; the mobility of small angle boundaries; the relative importance of the grain boundary plane, as compared with misorientation, in the recrystallization process; the relative contributions of twist versus tilt boundaries to recrystallization; and the relative contributions of special versus random boundaries to recrystallization are all important, especially for modelling. Also important are the experimental validation of the relation between grain boundary energy and grain boundary free volume (Fig. 44), the relationship between grain boundary mobility and grain boundary free volume needs to be elucidated experimentally, the effect of internal stresses on grain boundary motion, or more generally the interaction of grain boundaries with elastic stress fields, and the effect of inhomogeneities in deformation on grain boundary motion and the nature of defects left behind by the advancing grain boundary.

(21) Recently, a body of work has emerged which indicates that the performance of materials under a wide variety of conditions is affected by the grain boundary character distribution (GBCD) [352357,359]. Perhaps the most significant observation is that it is possible to exert control over the distribution of special and random grain boundaries through thermomechanical processing (including recrystallization), which turns this from a scientific curiosity to a potential tool for enhancing material performance [356,360-363]. It would be quite useful to develop a fundamental 
understanding of the mechanisms underlying the optimization of the GBCD, why the improvements in properties are apparently correlated with misorientation (not necessarily boundary plane), and the pervasiveness of this effect across many materials systems.

\section{Acknowledgements}

This paper was the result of the Workshop on Dislocation Reactions and the Formation of High-Angle Interfaces sponsored by the Division of Material Sciences, Basic Energy Science, US Department of Energy, in Monterey, California. One of the authors (WK) gratefully acknowledges technical discussions with $\mathrm{G}$. Campbell, A. Schwartz, L. Shvindlerman, G. Gottstein, C.L. Bauer, E. Lehockey, P. Lejcek, K. Merkle, V. Randle and D. Wolf. His work performed under the auspices of the Division of Materials Science of the Office of Basic Energy Sciences, US Department of Energy, and the Lawrence Livermore National Laboratory under contract No. W-7405-Eng-48. The authors also wish to express appreciation to other contributors to the workshop: T. Chandra, E. Evangelista, G. Gottstein, P. Kalu, T.G. Langdon, B. Randhakrishnan, T. Sakal, A. Schwartz, C. Tome, R. Vandermeer, K. Vecchio and J. Vetrano. Some of these participants were included as support for the BES-sponsored Center for Excellence in the Synthesis and Processing of Advanced Materials-Metals Forming Project (AS, BR, EE, MK, DH, HM, CT, JV).

\section{References}

[1] R.D. Doherty, G. Gottstein, J.R. Hirsch, W.B. Huttchinson, K. Lucke, E. Nes, P.J. Wilbrandt, in: J.S. Kallend, G. Gottstein (Eds.), Panel Discussion on Recrystallization Texture, ICOTOM 8, TMS, Warrendale, PA, 1988, p. 369.

[2] F.J. Humphreys, M. Hatherly, Recrystallization and Related Annealing Phenomena, Pergamon Press, Oxford, 1995.

[3] F. Haessner (Ed.), Recrystallization of Metallic Materials, 2nd ed., Rieder-Verlag, Stuttgart, 1978.

[4] V. Novikov, Grain Growth and Control of Microstructure and Texture in Polycrystalline Materials, CRC Press, Boca Raton, FL, 1996.

[5] J.W. Martin, R.D. Doherty, B. Cantor, Stability of Microstructure in Metallic Systems, 2nd ed., Cambridge University Press, Cambridge, 1997.

[6] R.D. Doherty, in: R.W. Cahn, P. Haasen (Eds.), Diffusive Phase Transformations in Physical Metallurgy, 4th ed., Elsevier, Amsterdam, 1996, p. 1369.

[7] S.P. Bellier, R.D. Doherty, Acta Metall. 25 (1977) 521-538.

[8] M. Volmer, A. Weber, Z. Phys. Chem. 119 (1926) 277.

[9] R. Becker, W. Doring, Ann. Phys. 24 (1934) 719.

[10] D. Turnbull, J.C. Fisher, J. Chem. Phys. 17 (1949) 71.

[11] D. Turnbull, in: F. Sietz, D. Turnbull (Eds.), Solid State Physics, vol. 3, Academic Press, New York, 1956, p. 226.

[12] J.W. Christian, The Theory of Transformations in Metals and Alloys, 2nd ed., Pergamon Press, Oxford, 1975.
[13] R.D. Doherty, Met. Sci. J. 8 (1974) 132-142.

[14] R.D. Doherty, in: F. Haessner (Ed.), Recrystallization of Metallic Materials, Dr. Rieder Verlag, Berlin, 1978, p. 23.

[15] R.W. Cahn, J. Inst. Met. 76 (1949) 121.

[16] M. Hatherly, in: T. Chandra (Ed.), Recrystallization '90, TMS, Warrendale, PA, 1990, p. 59.

[17] P. Haasen, Metall. Trans., 24a (1993) 1001.

[18] R.D. Doherty, S.F. Baumann, in: J.G. Morris, H.D. Merchant, E.J. Westerman, P.L. Morris (Eds.), Aluminum Alloys for Packaging, TMS, Warrendale, PA, 1993, p. 369.

[19] A.H. Cottrell, Prog. Metal Phys. (1953) 4.

[20] M. Ferry, F.J. Humphreys, Acta Mater. 44 (1996) 1293.

[21] F. Haessner, J. Schmidt, Acta Metall. Mater. 41 (1993) 1739.

[22] J. Hjelen, R. Orsund, E. Nes, Acta Metall. Mater. 39 (1991) 1377.

[23] M.G. Ardakani, F.J. Humphreys, Acta Metall. Mater. 42 (1994) 763.

[24] H. Hu, in: L. Himmel (Ed.), Recovery and Recrystallization of Metals, Wiley, New York, 1963, p. 331.

[25] P.A. Bech, P.R. Sperry, J. Appl. Phys. 21 (1950) 150.

[26] I.L. Dillamore, C.J.E. Smith, T.W. Watson, Met. Sci. J. 1 (1967) 49.

[27] P. Faivre, R.D. Doherty, J. Mater. Sci. 14 (1979) 897.

[28] A.R. Jones, B. Ralph, N. Hansen, Proc. R. Soc. A368 (1979) 345.

[29] R.D. Doherty, J. Szpunar, Acta Metall. 32 (1984) 1789.

[30] E. Woldt, F. Haessner, in: N. Hansen, D. Juul Jensen, Y.L. Liu, B. Ralph (Eds.), Microstructural and Crystallographic Aspects of Recrystallization, 16th Risø International Symposium on Materials Science, Risø National Lab., Roskilde, Denmark, 1995, p. 229.

[31] I. Samajdar, Ph.D. Thesis, Drexel University, 1994.

[32] R.D. Doherty, Scr. Metall. 19 (1985) 927.

[33] K. Lucke, Can. Met. Quart. 13 (1974) 261.

[34] D. Juul Jensen, in: N. Hansen, D. Juul Jensen, Y.L. Liu, B. Ralph (Eds.), Microstructural and Crystallographic Aspects of Recrystallization, 16th Risø International Symposium on Materials Science, Risø National Lab., Roskilde, Denmark, 1995, p. 119.

[35] R.D. Doherty, I. Samajdar, C.T. Necker, H.E. Vatne, E. Nes, in: N. Hansen, D. Juul Jensen, Y.L. Liu, B. Ralph (Eds.), Microstructural and Crystallographic Aspects of Recrystallization, 16 Risø International Symposium on Materials Science, Risø National Lab., Roskilde, Denmark, 1995, 1-23.

[36] B. Duggan, C.Y. Chung, in: H.J. Bunge (Ed.), Mater. Sci. Forum, ICOTOM 10 (1994) 157-163, 1765.

[37] B. Bay, N. Hansen, D.A. Hughes, D. Kuhlmann-Wilsdorf, Acta Metall. Mater. 40 (1992) 205-219.

[38] S. Panchanadeeswaran, R.D. Doherty, R. Becker, Acta Mater. 44 (1996) 1233.

[39] R. Becker, S. Panchanadeeswaan, Acta Metall. Mater. 43 (1995) 2701.

[40] A.J. Beaudoin, H. Mecking, U.F. Kocks, Philos. Mag. 73 (1996) 1503.

[41] J. Gil-Sevillano, P.V. Houtte, E. Aernoudt, Prog. Mater. Sci. 25 (1980) 69-412.

[42] C.S. Barrett, L.H. Levenson, Trans. AIME 137 (1940) 112127.

[43] R. Gotthardt, G. Hoscheck, O. Reinold, F. Haessner, Texture 1 (1972) 99.

[44] D.A. Hughes, in: N. Hansen, D. Juul Jensen, Y.L. Liu, B. Ralph (Eds.), Proc. 16th Risø International Symposium: Microstructural and Crystallographic Aspects of Recrystallization, Risø National Laboratory, Roskilde, Denmark, 1995, p. 63.

[45] B.J. Duggan, C.S. Lee, in: Y. Chu, Z. Liang, L. Peizi, L. Zhou, L. Zuo (Eds.), Eleventh International Conference on Textures and Materials, The Nonferrous Metals Society of China, Beijing, 1996, pp. $225-238$. 
[46] Proc. 1st International Conference on Low Energy Dislocation Structures, Mater. Sci. Eng. A81 (1986).

[47] Proc. 2nd International Conference onLow Energy Dislocation Structures, Mater. Sci. Eng. A113 (1989).

[48] Proc. 4th International Conference on Low Energy Dislocation Structures, Phys. Stat. Sol. (a) 149 (1995).

[49] Y. Chu, Z. Liang, L. Peizi, L. Zhou, L. Zuo (Eds.), Eleventh Internaitonal Conference on Textures of Materials ICOTOM 11, The Nonferrous Metals Society of China, Beijing, 1996.

[50] H. Weiland, Acta Metall. Mater. 40 (1992) 1083-1089.

[51] Q. Liu, N. Hansen, Phys. Stat. Sol. (a) 149 (1995) 187-199.

[52] B.L. Adams, S.I. Wright, K. Kunze, Metall. Trans. 24A (1993) 819-831.

[53] N.C. Krieger Lassen, D. Juul Jensen, Mater. Sci. Forum, $113-115$ (1993) 679-684.

[54] J.H. Driver, A. Skalli, M. Winterberger, Philos. Mag. 49 (1984) $505-524$.

[55] C.S. Lee, D.J. Duggan, Acta Metall. Mater. 41 (1993) 26912699.

[56] C.S. Lee, B.J. Duggan, R.E. Smallman, Acta Metall. Mater. 41 (1993) 2265-2270.

[57] J.A. Wert, Q. Liu, N. Hassen, Acta Metall. Mater. 43 (1995) 4153-4163.

[58] R. Becker, Modelling Simul. Mater. Sci. Eng. 3 (1995) $417-$ 435.

[59] A.J. Beaudoin, H.H. Mecking, U.F. Kocks, in: S.-F. Shen, P. Dawson (Eds.), Simulation of Materials Processing: Theory, Methods and Applications, Balkema, Rotterdam, 1995, p. 225.

[60] D. Kuhlmann-Wilsdirf, N. Hansen, Scr. Metall. Mater. 25 (1991) 1557-1562.

[61] P. Cizek, B.A. Parker, D.G. McCulloch, Mater. Sci. Eng. A194 (1995) 201-210.

[62] P. Cizek, B.P. Wynne, B.A. Parker, Scr. Mater. 35 (1996) $1129-1134$.

[63] G. Gottstein, R. Haasen, N. Hansen, Scripta Metall. Mater. Conf. Set No. 127 (1992) 1445.

[64] D.A. Hughes, N. Hansen, Metall. Trans. A 24A (1993) 20212037.

[65] D.A. Hughes, N. Hansen, Acta Mater. (1997) in press.

[66] J.H. Driver, in: N. Hansen, D. Juul Jensen, Y.L. Liu, B. Ralph (Eds.), Proc. 16th Risø International Symposium: Microstructural and Crystallographic Aspects of Recrystallization, Risø National Laboratory, Roskilde, Denmark, 1995, 25-36.

[67] N. Hansen, D. Juul Jensen, T. Leffers, B. Ralph (Eds.), Annealing Processes-Recovery, Recrystallization and Grain Growth, Risø National Laboratory, Roskilde, 1986.

[68] R.A. Vandermeer, in: T.R. McNelley ed. Proceeding of REX '96 The Third Inter. Conf. on Recrystallization and Related Phenomena, MIAS, Monterey, 1997, pp. 55-58.

[69] C.S. Barrett, Trans. AIME 137 (1940) 128.

[70] S. Kohara, M.N. Parthasarathi, P.A. Beck, Trans. AIME 212 (1961) 875.

[71] P.A. Beck, P.R. Sperry, H. Hu, J. Appl. Phys. 21 (1950) 420.

[72] B. Liebmann, K. Lücke, G. Masing, Z. Metallk. 47 (1956) 57.

[73] G. Gottstein, L.S. Shvindlerman, D.A. Molodov, in: T.R. McNelley ed. Proceeding of REX '96 The Third Inter. Conf. on Recrystallization and Related Phenomena, MIAS, Monterey, 1997, pp. 43-54.

[74] W.B. Hutchinson, Met. Sci. 8 (1974) 185.

[75] R.L. Everly, M. Hatherly, Texture 1 (1974) 183.

[76] A. Bardal, I. Lindseth, H.E. Vatne, E. Nes, in: N. Hansen et al. (Eds.), Proc. 16 Risø Int. Symp.: Microstructural and Crystallographic Aspects of Recrystallization, Risø, Roskilde, 1995, p. 261.

[77] R.A. Vandermeer, C.J. McHargue, Trans. AIME 230 (1964) 667.

[78] F. Haessner, K. Sztwiernia, Scr. Metall. Mater. 27 (1992) 1545.
[79] J.K. Mackenzie, Biometrika 45 (1958) 229.

[80] W.B. Hutchinson, L. Ryde, in: N. Hansen, D. Juul Jensen, Y.L. Liu, B. Ralph (Eds.), Proc. 16th Risø Int. Symp.: Microstructural and Crystallographic Aspects of Recrystallization, Risø, Roskilde, 1995, p. 105.

[81] D. Juul Jensen, Acta Metall. Mater. 43 (1995) 4117.

[82] D. Juul Jensen, in: T.R. McNelley ed. Proceeding of REX '96 The Third Inter. Conf. on Recrystallization and Related Phenomena, MIAS, Monterey, 1997, pp. 15-30.

[83] U. Schmidt, K. Lücke, Texture of Crystalline Solids 3 (1979) $85-112$.

[84] K. Lücke, Proc. ICOTOM-7, 7th Int. Conf. on Textures of Materials, Vol. 1, Holland, 1984, pp. 195-210.

[85] R.K. Ray, J.J. Jonas, M.P. Burton-Guillien, J. Savoie, Iron Steel Inst. Jpn. Intl. 34 (12) (1994) 927-942.

[86] B. Liebmann, K. Lücke, G. Masing, Z. Metallkde. 47 (1956) 57.

[87] G. Ibe, K. Lücke, Archiven fur Eisenhüttenwesen 39 (1968) $693-703$.

[88] L. Kestens, J.J. Jonas, in: T.R. McNelley ed. Proceeding of REX '96 The Third Inter. Conf. on Recrystallization and Relation Phenomena, MIAS, Monterey, 1997, pp. 109-122.

[89] J. Baczynski, J.J. Jonas, in: T.R. McNelley ed., Proceeding of REX ' 96 The Third Inter. Conference on Recrystallization and Related Phenomena, MIAS, Monterey, 1997, pp. 339-346.

[90] G. Gottstein, E. Brünger, L. Lütche, J. Fischer-Bühner, D. Ponge, Microstructural and Crystallographic Aspects of Recrystallization, Risø National Laboratory, Roskilde, Denmark, 1995, pp. 37-48.

[91] R.A. Vandermeer, D. Juul Jensen, Acta Metall. Mater. 42 (7) (1994) 2427-2436.

[92] R.A. Vandermeer, Microstructural and Crystallographic Aspects of Recrystallization, Risø National Laboratory, Roskilde, Denmark, 1995, pp. 193-213.

[93] W.B. Hutchinson, Mater. Sci. Forum 157-162 (1994) 19171928.

[94] W.B. Hutchinson, Acta Metall. 37 (4) (1989) 1047-1056.

[95] J. Kallend, Y. Huang, Met. Sci. 18 (1984) 381-385.

[96] C.T. Necker, R.D. Doherty, A.D. Rollett, Mater. Sci. Forum 157-162 (1994) 1021-1026.

[97] I. Dillamore, H. Katch, Met. Sci. 8 (1974) 73-83.

[98] A. Ridha, W. Hutchinson, Acta Metall. 30 (1982) 1929-1939.

[99] F.J. Humphreys, M.G. Ardakani, Acta Mater. 44 (1996) $2717-$ 2727.

[100] C. Necker, M.S. Thesis, Drexel University, 1992.

[101] H.E. Vatne, R. Shahani, E. Nes, Acta Mater. (1997) in press.

[102] B.J. Duggan, K. Lücke, G. Köhlhoff, C.S. Lee, Acta Metall. Mater. 41 (1993) 1921-1927.

[103] H.E. Vatne, O. Daaland, E. Nes, Mater. Sci. Forum 157-162 (1994) 1087-1094.

[104] A.D. Rollett, W.W. Mullins, Scr. Metall. Mater. 36 (1997) 975-980.

[105] G. Gottstein, F. Schwarzer, Mater. Sci. Fourm 94-96 (1992) $187-208$.

[106] R.C. Sun, C.L. Bauer, Acta Metall. 18 (1970) 639-647.

[107] B.L. Adams, D.J. Spolovitz, G.S. Grest, P.S. Sahni, Mater. Sci. Forum 204-206 (1996) 179-190.

[108] M.P. Anderson, Acta Metall. 32 (1984) 783-791.

[109] G. Abbruzzese, K. Lücke, Acta Metall. 34 (1986) 905-914.

[110] H.J. Bunge, U. Köhler, Scr. Metall. Mater. 27 (1992) 15391543.

[111] F.J. Humphreys, in: T.R. McNelley ed., Proceedings of REX '96 The Third Inter. Conference on Recrystallization and Related Phenomena, MIAS, Monterey, 1991, pp. 3-15.

[112] K. Mahin, K. Hanson, J. Morris, Acta Metall. 28 (1980) 443.

[113] H. Frost, C. Thompson, Acta Metall. 35 (1987) 529-540.

[114] T. Furu, K. Marthinsen, E. Nes, Mater. Sci. Tech. 6 (1990) 1093. 
[115] D. Juul Jensen, Scr. Metall. Mater. 27 (1992) 1551-1556.

[116] F.J. Humphreys, Scr. Meall. Mater. 27 (1992) 1557-1562.

[117] V.E. Fradkov, L.S. Shvindlerman, D. Udler, Scr. Metall. 19 (1985) 1285-1290.

[118] H.J. Frost, C.V. Thompson, D.T. Walton, Acta Metall. Mater. 40 (4) (1992) 779-793.

[119] A.D. Rollett, E.A. Holm, in: T.R. McNelley ed., Proceedings of REX '96 The Third Inter. Conference on Recrystallization and Related Phenomena, MIAS, Monterey, 1997, pp. 31-42.

[120] H.W. Hesselbarth, I.R. Göbel, Acta Metall. 39 (9) (1991) 2135.

[121] D.J. Srolovitz, G.S. Grest, M.P. Anderson, Acta Metall. 34 (1986) 1833-1845.

[122] A.D. Rollett, D.J. Srolovitz, M.P. Anderson, R.D. Doherty, Acta Metall. 40 (1992) 3475-3495.

[123] P. Tavernier, J.A. Szpunar, Acta Metall. Mater. 39 (4) (1991) $557-567$.

[124] G.N. Hassold, E.A. Holm, D.J. Srolovitz, Scr. Metall. Mater. 24 (1990) 101-106.

[125] R.D. Doherty, K. Li, M.R. Anderson, A.D. Rollett, D.J. Srolovitz, Recrystallization '90, TMS-AIME, Warrendale, PA, 1990, pp. 129-150.

[126] L.Q. Chen, Scr. Metall. Mater. 32 (1) (1995) 115-120.

[127] G. Sarma, B. Radhakrishnan, T. Zacharia, in: T.R. McNelley ed., Proceedings of REX '96 The Third Inter. Conference on Recrystallization and Related Phenomena, MIAS, Monterey, 1997, pp. $555-562$.

[128] E. Nes, W.B. Hutchinson, in: Bilde-Sorensen (Eds.), Proc. 10th Int. Risø Symp., Risø, Denmark, 1989, p. 233.

[129] T. Furu, K. Marthinsen, E. Nes, in: Fuentes and Gill Sevillano (Eds.), Recrystallization '92, 1992, p. 41.

[130] F.J. Humphreys, Proc. 11th Int. Conf. on Textures of Materials ICOTOM 11, Nonferrous Metals Society of China, Beijing, 1996.

[131] F.J. Humphreys. M. Ferry, C. Johnson, P. Paillard, Proc. 16th Risø Int. Symp.: Microstructural and Crystallographic Aspects of Recrystallization, Risø National Laboratoryk, Roskilde, Denmark, Denmark, 1995, p. 87.

[132] E. Hormbogen, U. Köster, in: F. Haessner, Dr. Riederer (Eds.), Recrystallization of Metallic Materials, Verlag, Berlin, 1978, p. 159.

[133] O. Engler, J. Hirsch, K. Lücke, Acta Metall. Mater. 43 (1995) 121.

[134] O. Daaland, E. Nes, Acta Mater. 44 (1996) 1413.

[135] H.E. Vatne, O. Engler, E. Nes, Proc. 10th Int. Conf. on Textures of Metals ICOTOM 10, Clauthal, 1994, p. 1501.

[136] K. Lücke, O. Engler, Mater. Sci. Tech. 6 (1990) 1113.

[137] F.J. Humphreys, D. Juul Jensen, Proc. 7th Int. Risø Symp. Risø, Denmark, 1986, p. 93.

[138] H.M. Chan, F.J. Humphreys, Acta Metall. 32 (1984) 235.

[139] F.J. Humphreys, I. Brough, in: T.R. McNelley ed., Proceedings of REX '96 The Third Inter. Conference on Recrystallization and Related Phenomena, MIAS, Monterey, 1997, pp. 315-322.

[140] A.W. Bowen, M.G. Ardakani, F.J. Humphreys, in: Clausthal (Ed.), Proc. ICOTOM 10, vol. 1, Bunge, 1994, p. 919.

[141] M.J. Luton, C.M. Sellars, Acta Metall. 17 (1969) 1033.

[142] T. Sakai, J.J. Jonas, Acta Metall. 32 (1984) 189.

[143] L.S. Tóth, J.J. Jonas, Scr. Metall. Mater. 27 (1992) 359.

[144] J. Baczynski, J.J. Jonas, Proc. 11th Int. Conf. on Textures of Materials ICOTOM-11, Nonferrous Metals Society of China, Beijing, 1996, p. 387.

[145] H.J. McQueen, E. Evangelista, J. Bowles, G. Crawford, Met. Sci. 18 (1984) 395.

[146] I. Kozasu, C. Ouchi, T. Sampei, T. Okits, Microalloying '75, Proc. Int. Symp. on HSLA Steels, Union Carbide, New York, 1977, p. 120.

[147] M.R. Barnett, J.J. Jonas, ISIJ Int. 37 (1997) 697.

[148] T. Watanabe, Mater. Sci. Forum 94-96 (1992) 209.
[149] L. Kestens, J.J. Jonas, Metall. Mater. Trans. 27A (1996) 155.

[150] T. Urabe, J.J. Jonas, ISIJ Int. 34 (1994) 435.

[151] D. Wolf, Philos. Mag. B. 59 (1989) 667.

[152] Y.B. Park, D.N. Lee, G. Gottstein, Proc. 11th Int. Conf. on Textures of Materials ICOTOM-11, Nonferrous Metals Society of China, Beijing, 1996, p. 531.

[153] D.N. Lee, Scr. Metall. Mater. 32 (1995) 1689.

[154] G. Gottstein, H. Mecking, D. Zabardjadi, Proc. Int. Conf. Strength of Metals and Alloys 4, Nancy, Vol. III, 1976, p. 1126.

[155] Ph. Bocher, Ph.D. Thesis, McGill University, Montreal, Canada, 1997.

[156] L.Pl Karjalainen, Pl Kantanen, T.M. Maccagno, J.J. Jonas, THERMEC '97, Wollongong, Australia, 1997, in press.

[157] J.J. Jonas, Mater. Sci. Eng. A184 (1994) 155.

[158] P.D. Hodgson, R.E. Gloss, G.L. Dunlop, Proc. 32nd Mechanical Working and Steel Processing Conf. ISS-AIME, Warrendale, PA, 1991, p. 527.

[159] J.J. Jonas, I. Weiss, Met. Sci. 13 (1979) 238.

[160] K. Minami, F. Siciliano Jr., T.M. Maccagno, J.J. Jonas, ISIJ Int. 36 (1996) 1507.

[161] J.A. Hines, K.S. Vecchio, Acta Mater. 45 (1997) 635.

[162] M. Militzer, W.P. Sun, J.J. Jonas, Acta Metall. Mater. 42 (1994) 133.

[163] E. Hornbogen, Metall. Trans. A 10A (1979) 947-972.

[164] H.J. McQueen, J.J. Jonas, Treatise on Materials Science and Technology, vol. 6, Academic Press, New York, 1975, pp. 393-493.

[165] E. Nes, Mater. Sci. 13 (1978) 2052-2055.

[166] E. Nes, Met. Sci. J. 13 (1979) 211-220.

[167] R.D. Doherty, G. Gottstein, J. Hirsch, W.B. Hutchinson, K. Lucke, E. Nes, P.J. Wilbrandt, in: J.S. Kallend, G. Gottstein (Eds.), Proc. ICOTOM8, TMS, Warrendale, PA, 1988, 563572.

[168] E. Nes, in: B. Baudelet, M. Surey (Eds.), Superplasticité, Centre National de la Recherche Scientifique, Paris, 1985, pp. 7.17.14 .

[169] S.J. Hales, T.R. McNelley, H.J. McQueen, Metall. Trans. A 22A (1991) 1037-1047.

[170] J. Dutkiewicz, Metall. Trans. A 8a (1977) 751-761.

[171] R. Grimes, NATO-AGARD Lecture Series No. 168, 1988, pp. $8.1-8.16$

[172] B.M. Watts, M.J. Stowell, B.L. Baike, D.G.E. Owen, Met. Sci. J. 10 (1976) 189-197.

[173] R.H. Bricknell, J.W. Edington, Acta Metall. 27 (1979) $1303-$ 1311.

[174] T.R. McNelley, M.E. McMahon, J. Met. 48 (2) (1996) 48-51.

[175] T.R. McNelley, M.E. McMahon, Metall. Trans. A 27A (1996) 2252-2262.

[176] H.G. McQueen, O. Knustad, N. Ryum, J.K. Solberg, Scr. Metall. 19 (1985) 73.

[177] J.K. Solberg, H.J. McQueen, N. Ryum, E. Nes, Philos. Mag. A. 60 (1989) 447-471.

[178] H.J. McQueen, J.K. Solberg, N. Ryum, E. Nes, Philos. Mag. A. 60 (1989) $473-485$.

[179] D. Hardwick, W.J. McG. Tegart, J. Inst. Met. 90 (1961-1962) 17.

[180] J.R. Cotner, W.J. McG. Tegart, J. Inst. Met. 97 (1969) 73.

[181] M.E. Kassner, N.Q. Nguyen, G.A. Henshall, H.J. McQueen, Mater. Sci. Eng. A132 (1991) 97.

[182] S.L. Kuzmin, V.A. Likachev, M.M. Myshlyaev, A.N. Yu, O.N. Senkov, Scr. Metall. 12 (1978) 735.

[183] M.M. Myshlyaev, O.N. Senkov, V.A. Kikhachev, in: H.J. McQueen, J.-P. Baillon, J.I. Dickson, J.J. Jonas, M.G. Akben (Eds.), Strength of Metals and Alloys, Pergamon, Oxford, 1985, p. 841.

[184] M.E. Kassner, M.E. McMahon, Metall. Trans. A81 (1987) 835-846. 
[185] C. Perdrix, Y.M. Perrin, F. Montheillet, Mem. Sci. Rev. Metall. 78 (1981) 309-320 (in French); English translation, Lawrence Livermore National Laboratory Report UCIR-1623, 1986.

[186] M.E. Kassner, J.J. Oldani, Proc. IX Inter-American Conf. on Material Technology, Editorial Universitaria, Santiago, Chile, 1987, p. 219.

[187] V.A. Likhachev, M.M Myshlyaev, O.N. Senkov, Laws of the Superplastic Behavior of $\mathrm{Al}$ in Trosion, Institute of Solid State Physics, Chernogolovka, 1981 (in Russian); English tranlation Lawrence Livermore National Laboratory Reprot UCIR-1681, 1987.

[188] M.E. Kassner, M.M. Myshlyaev, H.J. McQueen, Mater. Sci. Eng. A108 (1989) 45-61.

[189] M.E. Kassner, Metall. Trans. A 20 (1989) 2182-2185.

[190] H.J. McQueen, M.E. Kassner, in: H.C. Heikkenen, T.R. McNelley (Eds.), Superplasticity in Aerospcae, AIME, Warrendale, PA, 1988, pp. 77-96.

[191] D. Caillard, J.L. Martin, Acta Metall. 30 (1982) 791.

[192] F.J. Humphreys, in: R.C. Gifkins (Ed.), Strength of Metals and Alloys, Pergamon Press, Oxford, 1982, p. 625.

[193] G.A. Henshall, M.E. Kassner, H.J. McQueen, Metall. Trans. A23 (1992) 881-889.

[194] H.J. McQueen, E. Evangelista, M.E. Kassner, Z. Metall. 82 (1991) 336-345.

[195] H. Yamagata, Scr. Metall. Mater. 27 (1992) 1157-1162.

[196] H. Yamagata, Scr. Metall. Mater. 30 (1994) 411-416.

[197] M.E. Kassner, J. Pollard, E. Evangelista, E. Cerri, Acta Metall. Mater. 42 (1994) 3223.

[198] M. Ueki, S. Horie, T. Nakamura, in: E.A. Starke, T.H. Sanders (Eds.), Aluminum Alloys Physical and Mechanical Properties, EMAS Warley, 1986, p. 419.

[199] M. Ueki, S. Horie, T. Nakamura, Mater. Sci. Tech. 3 (1987) 329.

[200] E. Usui, T. Inaba, N. Shiman, Z. Metallkde. 77 (1986) 684.

[201] E. Usui, T. Inaba, N. Shiman, Z. Metallkde. 77 (1986) 179.

[202] M.E. Kassner, in: H.J. McQueen, E.V. Koenopleva, N.D. Ryan (Eds.), Hot Workability of Steels and Light Alloys-Composites, Can. Inst. Metallurgist, Montreal, 1996, p. 3.

[203] I. Poschmann, H.J. McQueen, Scr. Metall. It. Mater. 35 (1991) $1123-1128$.

[204] C.G. Schmidt, C.M. Young, B. Walser, R.H. Klundt, D.D. Sherby, Metall. Trans. 13A (1982) 447.

[205] M.A. Meyers, J.C. La Salvia, V.F. Nesterenks, B.K. Kad, in: T.R. McNelley ed., Proceedings of REX ' 96 The Third Inter Conference on Recrystallization and Related Phenomena, MIAS, Mortality, 1997, pp. 279-286.

[206] W. Blum, in: R.W. Arsenault, D. Cole, T. Gross, G. Kostorz, P. Liaw, S. Parameswaran, H. Sizek (Eds.), The Johannes, Weertman Symp., The Metals and Materials Society, Warrendale, PA, 1996, 103-107.

[207] W.D. Nix, B. Ilschner, in: G. Haasen, P. Haasen, V. Gerold, G. Kostorz (Eds.), Strength of Metals and Alloys, Proc. ICSMA 5, Pergammon Press, Oxford, 1979, pp. 1503-1530.

[208] C.R. Barrett, W.D. Nix, Acta Metall. 13 (1965) 1247-1258.

[209] J. Weertman, in: B. Wilshire, D.R.J. Owen (Eds.), Creep and Fracture of Engineering Materials and Structures, Pineridge Press, Swansea, 1984, pp. 1-14.

[210] W. Blum, in: T.G. Langdon, H.D. Merchant, J.G. Morris, M.A. Zaidi (Eds.), Hot Deformation of Al Alloys, TMSAIME, Warrendale, PA, 1991, pp. 181-210.

[211] T.G. Langdon, in: R.C. Gifkins (Ed.), Strength of Metals and Alloys, Proc. ICSMA 5, Pergamon Press, Oxford, 1982, pp. $1105-1120$.

[212] D. Caillard, J.L. Martin, Rev. Phys. Appl. 22 (1987) 169-183.

[213] I. Poschmann, H.J. McQueen, Phys. Stat. Sol. A149 (1995) 341-348.

[214] I. Poschmann, H.J. McQueen, Mater. Sci. Eng. (1996), in press.
[215] J. Grewen, G. Wasserman, Metall. 12 (1958) 523-525.

[216] H.J. McQueen, J.J. Jonas, J. Appl. Metal Working 3 (1984) 233-241; 410-420.

[217] H.J. McQueen, D.L. Bourell, J. Met. 39 (7) (1987) 28-35.

[218] H.J. McQueen, in: T.G. Langdon, H.D. Merchant (Eds.), Hot Deformation of Aluminum Alloys, TMS-AIME, Warrendale, PA, 1991, pp. 31-54.

[219] H.J. McQueen, H. Mecking, Creep and Fracture of Engineering Materials and Structures, Pinridge Press, Swansea, 1984, pp. $169-184$.

[220] H.J. McQueen, E. Evangelista, Czech. J. Phys. B38 (1988) $359-372$.

[221] H.J. McQueen, J. Mater. Proc. Tech. 37 (1993) 3-36.

[222] H.J. McQueen, E. Evangelista, N. Jin, M.E. Kassner, Metall. Trans. 26A (1995) 1757-1766.

[223] H.J. McQueen, J.E. Hockett, Met. Trans. 1 (1970) 2997-3004.

[224] H.J. McQueen, W. Blum, Q. Zhu, V. Demuth, in: J.J. Jonas, T.R. Bieler, K.J. Bowman (Eds.), Advances in Hot Deformation Textures and Microstrucures, TMS-AIME, Warrendale, PA, 1993, pp. 235-250.

[225] W. Blum, H.J. McQueen, Mater. Sci. Forum 217-222 (1996) $31-42$.

[226] W. Blum, Q. Zhu, R. Merkel, H.J. McQueen, Z. Metalkde. 87 (1996) 341-348.

[227] Q. Zhu, W. Blum, H.J. McQueen, Mater. Sci. Forum 217-222 (1996) $1169-1174$.

[228] S. Gourdet, E.V. Konopleva, H.J. McQueen, F. Montheillet, Mater. Sci. Forum 217-222 (1996) 441-446.

[229] H.J. McQueen, J.J. Jonas, in: C.Q. Chen (Ed.), Aluminum Alloys '90, Proc. ICAA2, Beijing, 1990, pp. 727-742.

[230] H.J. McQueen, E. Evangelista, N. Jin, M.E. Kassner, in: J.J Jonas, T.R. Bieler, K.J. Bowman (Eds.), Advances in Hot Deformation Textures and Microstructures, TMS-AIME, Warrendale, PA, 1993, pp. 251-266.

[231] W. Blum, Q. Zhu, R. Merkel, H.J. McQueen, Mater. Sci. Eng. A205 (1996) 23-30.

[232] I. Guttierez, M. Fuentes, in: T. Chandra (Ed.), Recrystallization '90, TMS-AIME, Warrendale, PA, 1990, 807-812.

[233] H.J. McQueen, N.D. Ryan, R. Zaripova, K. Farkhutdinov, in Proc. 37 Mech. Working and Steel Processing Conf., Iron Steel Soc., AIME, Warrendale, PA, 1996, pp. 883-894.

[234] H.J. McQueen, M.E. Kassner, in: T.T. McNelley, H.C. Heikkenen (Eds.), Superplasticity in Aerospcae II, TMS-AIME, 1991, pp. 189-206.

[235] E. Weckert, W. Blum, in: H.J. McQueen, J.J. Jonas, H.K. Akben, J.-P. Bailon (Eds.), Strength of Metals and Alloys, Proc. ICSMA 7, vol. 1, Pergamon Press, Oxford, 1985, pp. $773-778$.

[236] H.J. McQueen, W. Blum, in: T.R. McNelley (Ed.), Recrystallization and Related topics (Proc. 3rd Intnl. Conf. REX 96), in press.

[237] S. Straub, W. Blum, in H.J. McQueen, L.V. Konopleva, N.D. Ryan (Eds.), Hot Workability of Steels and Light Alloys-Composites, Met. Soc. CIM, Montreal, 1996, pp. 189-204.

[238] M. Meier, Q. Zhu, W. Blum, Z. Metallkde. 84 (1993) 263.

[239] Q. Zhu, W. Blum, in: Y. Husoi, H. Yoshinaga, H. Oikawa, K. Maruyama (Eds.), Aspects of High Temperature Deformation and Fracture in Crystalline Materials, JIMIS-7, The Japan Institute of Metals, 1993, pp. 649-656.

[240] R. Merkel, Unterschungen der Mikrostruktur von Al Legierungen nach Umformung in Torsionversuchen, Materials Science, University of Erlangen, Nurenberg, 1994.

[241] H.J. McQueen, N. Ryum, Scand. J. Metall. 14 (1985) 183-194.

[242] E.V. Konopleva, H.J. McQueen, E. Evangelista, Mater. Characterization 34 (1995) 251-264.

[243] F.J. Humphreys, M.R. Drury, in: T. Sheppard (Ed.), Aluminum Technology, Inst. Metals, London, 1986, pp. 191-196. 
[244] H.J. McQueen, N.D. Ryan, E.V. Konopleva, X. Xia, Can. Metal. Quart. 34 (1995) 219-229.

[245] H.J. McQueen, W.A. Wong, J.J. Jonas, Can. J. Phys. 45 (1967) $1225-1239$.

[246] H.J. McQueen, W. Blum, S. Straub, M.E. Kassner, Scr. Metall. Mater. 28 (1993) 1299-1304.

[247] D.A. Hughes, in: J.G. Morris, H.D. Merchant, E.J. Westerman, P.L. Morris (Eds.), Al Alloys for Packaging II, TMS-AIME, Warrendale, PA, 1996, pp. 129-144.

[248] G.R. Canova, S. Shrivastava, J.J. Jonas, C. G'Sell, in: J.R. Newby, B.A. Niemeier (Eds.), Formability of Metallic Materials-2000 AD. STP753, ASTM, Philadelphoa, 1982, pp. 182210.

[249] C.L. Maurice, M.C. Theyssier, J.H. Driver, in: J.J. Jonas, T.R. Bieler, K.J. Bowman (Eds.), Advance in Hot Deformation Textures and Microstructures, TMS-AIME, Warrendale, PA, 1995, pp. 411-425.

[250] M.C. Theyssier, B. Chenal, J.H. Driver, N. Hansen, Phys. Stat. Sol. A149 (1995) 367-378.

[251] H.J. McQueen, H. Mecking, Z. Metallkde. 78 (1987) 387-395.

[252] K.V. Gow, R.W. Cahn, Acta Metall. 1 (1954) 238-241.

[253] E. Hirosowa, J. Japan. Inst. Met. 92 (1963) 78-81.

[254] A. Plumtree, G. Deep, Met. Tech. 4 (1977) 1-5.

[255] E. Aernoudt, H.P. Stuwe, Z. Metallkde. 61 (1970) 128.

[256] S.R. Goodman, H. Hu, Trans. Met. Soc. AIME 230 (1964) 1413-1419; 233 (1965) 103-110.

[257] E.L. Brown, A.J. Deardo, Met. Trans. 12A (1981) 39.

[258] H.J. McQueen, Trans. Jpn. Inst. Metalls 9 (Suppl.) (1968) $170-177$.

[259] D.A. Hughes, N. Hansen, in: J.J. Jonas, T.R. Bieler, K.J. Bowman (Eds.), Advances in Hot Deformation Textures and Microstructure, TMS-AIME, Warrendale, PA, 1995, pp. 427444.

[260] N. Hansen, D. Juul Jensen, in: T.G. Langdon, H.D. Merchant, J.G. Morris, M.A. Zaidi (Eds.), Hot Working of Al Alloys, TMS-AIME, Warrendale, PA, 1991, pp. 3-20.

[261] D.A. Hughes, Y.L. Liu, in: T.G. Langdon, H.D. Merchant, J.G. Morris, M.A. Zaidi (Eds.), Hot Working of Al Alloys, TMS-AIME, Warrendale, PA, 1991, pp. 21-30.

[262] T. Hasegawa, T. Yakou, U.F. Kocks, Acta Metall. 27 (1979) 1705; 30 (1982) 235.

[263] N.D. Ryan, H.J. McQueen, Mater. Sci. Tech. 7 (1991) 815828.

[264] N.D. Ryan, H.J. McQueen, Can. Met. Quart. 30 (1991) 113124.

[265] E. Evangelista, H.J. McQueen, N.D. Ryan, J. Bowles, Stainless Steel, 1991, Iron Steel Inst. Japan, Tokyo, 1991, pp. 842-849.

[266] N.D. Ryan, H.J. McQueen, J. Mater. Proc. Tech. 36 (1993) $103-123$.

[267] H.J. McQueen, Mater. Sci. Eng. A101 (1987) 149-160.

[268] I. Poschmann, H.J. McQueen, Z. Metallkde. 88 (1997) 14-22.

[269] H.J. McQueen, G. Avramovic-Cingara, P. Sakaris, A. Cingara, Proc. 3rd Int. SAMPLE Metals Conf., 1992, pp. M192-M206.

[270] G. Avramovic-Cingara, K. Conrod, A. Cingara, H.J. McQueen, Proc. Int. Symp. on Light Metals Processing and Application, vol. 7, Met. Soc. Tech., 1993, pp. 495-510.

[271] I. Poschmann, H.J. McQueen, Z. Metallkde. 87 (1996) 349356.

[272] H.J. McQueen, N.D. Ryan, in: N.D. Ryan, A. Brown, H.J. McQueen (Eds.), Strip Casting and Hot and Cold Working, Met. Soc. CIM, Montreal, 1993, pp. 91-106.

[273] H.J. McQueen, S. Yue, N.D. Ryan, E. Fry, L.A. Dobrzanski (Ed.), Advanced Materials and Technologies, Silesian Tech. Univ., Gliwice, Poland, 1995, pp. 295-332.

[274] H.J. McQueen, S. Yue, N.D. Ryan, E. Fry, J. Mater. Proc. Tech. 53 (1995) 293-310.
[275] A. Berger, P.J. Wilbrandt, P. Haasen, Acta Metall. 31 (1983) $1433-1443$.

[276] H.J. McQueen, E. Evangelista, N.D. Ryan, in: T. Chandra (Ed.), Recrystallization ' 90 in Metals and Materials, AIME, Warrendale, PA, 1990, pp. 89-100.

[277] H. Mecking, G. Gottstein, in: F. Haessner (Ed.), Recrystallization of Metallic Materials, Dr. Reidereer Verlag, Berlin, 1977, pp. 195-212.

[278] C.M. Sellars, Philos. Trans. R. Soc. A288 (1978) 147-158.

[279] N.D. Ryan, H.J. McQueen, High Temp. Tech. 8 (1990) 185200.

[280] N.D. Ryan, H.J. McQueen, E. Evangelista, Mater. Sci. Eng. 81 (1986) 259-272.

[281] H.J. McQueen, Y. Cui, B. Li, Q. Meng, N.D. Ryan, in: N.D. Ryan, A. Brown, H.J. McQueen (Eds.), Strip Casting, Hot and Cold Working of Stainless Steels, Met. Soc. CIMM, Montreal, 1993, pp. 181-192.

[282] H.J. McQueen, N. Jin, N.D. Ryan, Mater. Sci. Eng. A190 (1995) 43-53.

[283] H.J. McQueen, S. Bergerson, Met. Sci. J. 6 (1972) 25-29.

[284] F.E. White, Rev. Met. 63 (1966) 991-998.

[285] R. Lombry, G. Rossard, B.J. Thomas, Rev. Met. CIT 78 (1981) 975-988.

[286] D.L. Bourell, H.J. McQueen, J. Mater. Shaping Tech. 5 (1987) $53-73$.

[287] G. Glover, C.M. Sellars, Metall. Trans. 4A (1973) 765-775.

[288] M.E. Kassner, H.J. McQueen, J. Pollard, E. Evangelista, E. Cerri, Scr. Metall. Mater. 31 (1994) 1331-1336.

[289] T. Matuszewski, H.J. McQueen, P.M. Machmeier, Metall. Mater. Trans. 25A (1994) 827-837.

[290] M. Richert, H.J. McQueen, in: H.J. McQueen, E.V. Konopleva, N.D. Ryan (Eds.), Hot Workability of Steels and Light Alloys-Composites, Met. Soc. CIM, Montreal, 1996, pp. 15-26.

[291] F. Schuh, M. Von Heimendahl, Z. Metallkde. 65 (1974) 356.

[292] H. Ahlborn, E. Hornbogen, U. Koster, J. Mater. Sci. 4 (1969) 944-950.

[293] B.M. Watts, M.J. Stowell, B.L. Bakie, D.G.E. Owen, Met. Sci. 10 (1976) 189-197, 198-205.

[294] H. Gleiter, B. Chalmers, High-Angle Grain Boudaries, Pergamon Press, Oxford, New York, 1972.

[295] A.P. Sutton, R.W. Balluffi, Interfaces in Crystalline Materials, Clarendon Press, Oxford University Press, Oxford, New York, 1995.

[296] K.L. Merkle, D. Wolf, MRS Bull. 15 (1990) 42.

[297] H. Grimmer, W. Bollmann, D.H. Warrington, Acta Cryst. 30A (1974) 197.

[298] V. Randle, The Measurement of Grain Boundary Geometry, IOP, London, 1993.

[299] D.G. Brandon, Acta Metall. 14 (1966) 1479.

[300] W.T. Reed, W. Shockley, Phys. Rev. 78 (1950) 275.

[301] B.L. Adams, J. Zhao, H. Grimmer, Acta Cryst. A46 (1990) 620.

[302] G. Palumbo, K.T. Aust, Acta Metall. Mater. 38 (1990) 2343.

[303] P.H. Pumphrey, in: G.A. Chadwick, D.A. Smith (Eds.), Grain Boundary Structure and Properties, Academic Press, New York, 1976, p. 139.

[304] Y. Ishida, M. McLean, Philos. Mag. 27 (1973) 1125.

[305] G. Palumbo, K.T. Aust, Can. Metall. Quart. 34 (1995) 165.

[306] V. Rybin, A.A. Zisman, Poverhnost 1 (1982) 87.

[307] L. Shvindlerman, B. Straumal, Acta Metall. 33 (1985) 1735.

[308] Y. Yoshitomi, S. Suzuki, S.T. Ueda, H. Tsurekawa, Nakashima H. Yoshinaga, Scr. Metall. Mater. 32 (1995) 1067.

[309] T. Muschik, S. Hofmann, W. Gust, B. Predel, Appl. Surf. Sci. 37 (1989) 439.

[310] B.D. Powll, D.P. Woodruff, Philos. Mag. 34 (1976) 169.

[311] T. Watanabe, T. Murakami, S. Karashima, Scr. Metall. 12 (1978) 361. 
[312] S. Hofmann, Vacuum 40 (1990) 9.

[313] D. Bouchet, B. Aufray, L. Priester, J. Phys. 49 (1988) C5.

[314] G. Gottstein, D.A. Molodov, U. Czubayko, L.S. Shvindlerman, J. Phys. IV (C3) 5 (1994) 89.

[315] J.E. Burke, D. Turnbull, Prog. Met. Phys. (1952) 234.

[316] H. Fukutomi, Trans. Jpn. Inst. Met. 22 (1981) 633.

[317] H. Gleiter, S. Mahajan, K.J. Bachmann, Acta Metall. 28 (1980) 1603.

[318] G. Gottstein, U. Czubayko, D.A. Molodov, L.S. Shvindlerman, W. Wundrlich, Mater. Sci. Forum 204-206 (1996) 99.

[319] L.S. Shvindlerman, U. Czubayko, G. Gottstein, D.A. Molodov, Mater. Sci. Forum 204-206 (1996) 45.

[320] D.A. Molodov, U. Czubayko, G. Gottstein, L.S. Shvindlerman, Mater. Sci. Forum 207-209 (1996) 133.

[321] D.A. Molodov, U. Czubayko, G. Gottstein, L.S. Shvindlerman, B. Straumal, W. Gust, Philos. Mag. Lett. 72 (1995) 361.

[322] D.A. Molodov, U. Czubayko, G. Gottstein, L.S. Shvindlerman, Scr. Metall. Mater. 32 (1995) 529.

[323] W. Lojkowski, D.A. Molodov, G. Gottstein, L.S. Shvindlerman, Mater. Sci. Forum 207-209 (1996) 537.

[324] J. Sommer, C. Herzig, T. Muschik, W. Gust, Acta Metall. Mater. 43 (1995) 1099.

[325] S. Porowski, W. Lojkowski, D.A. Molodov, L.S. Shvindlerman, G. Gottstein, High-Pressure Science and Technology1993, Joint International Association for Research and Advancement of High Pressure Science and Technology and American Physical Society Topical Group on Shock Compression of Condensed Matter Conference, Colorado Springs, CO, 1993, p. 1217.

[326] S. Tsurekawa, T. Ueda, K. Ichikawa, N. Nakashima, Y. Yoshitomi, H. Yoshinaga, Mater. Sci. Forum 204-206 (1995) 221.

[327] D.A. Molodov, C.V. Kopetskii, D.S. Shvindlerman, Dokl. Akad. Nauk SSSR 260 (1981) 1111.

[328] D.A. Molodov, U. Czubayko, G. Gottstein, L.S. Shvindlerman, Mater. Sci. Forum 217-222 (1996) 523.

[329] V. Randle, M. Caul, J. Fiedler, J. Microscopy Soc. Am. (1997) submitted.

[330] J. Gastaldi, C. Jourdan, G. Grange, C.L. Bauer, Phys. Stat. Sol. A 109 (1988) 403.

[331] J. Gastaldi, C. Jourdan, G. Grange, Philos. Mag. A, Phys. Condens. Matter Defects Mech. Prop. 57 (1988) 971.

[332] J.W. Rutter, K.T. Aust, Acta Metall. 13 (1965) 181.

[333] K.T. Aust, J.W. Rutter, Trans. AIME 215 (1959) 119.

[334] K.T. Auts, J.W. Rutter, Trans. AIME 215 (1959) 820.

[335] D.A. Molodov, B.B. Straumal, L.S. Shvindlerman, Scr. Metall. 18 (1984) 207.

[336] R. Viswanathan, C.L. Bauer, Acta Metall. 21 (1973) 1099.

[337] A.N. Aleshin, V.Y. Aristov, B.S. Bokstein, L.S. Shvindlerman, Phys. Stat. Sol. A 45 (1978) 359.
[338] V.Y. Aristov, V.L. Mirochnik, L.S. Shvindlerman, Sov. Phys. Solid State 18 (1976) 137.

[339] E.M. Fridman, C.V. Kopetskii, L.S. Shvindlerman, Z. Metallkde. 66 (1975) 533.

[340] L.S. Shvindlerman, V.G. Sursaeva, V.P. Yashnikov, R.G. Faulker, Interface Sci. 2 (1994) 153.

[341] L.S. Shvindlerman, E.M. Fridman, Sov. Phys. Solid State 15 (1974) 2466.

[342] G. Gottstein, L.S. Shvindlerman, Scr. Metall. Mater. 27 (1992) 1515.

[343] P. Lejcek, J. Adamek, J. Phys. IV, Colloq. 5 (1994) 107.

[344] H. Nakashima, T. Ueda, S. Tsurekawa, K. Ichikawa, H. Yoshinaga, Tetsu To Hagane 82 (1992) 238.

[345] K. Ichikawa, T. Ueda, S. Tsurekawa, Y. Yoshitomi, Y. Matsuo, K. Kawasaki, H. Nakashima, H. Yoshinaga, Tetsu To Hagan 82 (1996) 471.

[346] D.A. Molodov, J. Swiderski, G. Gottstein, W. Lojkowski, L.S. Shvindlerman, Acta Metall. 42 (1994) 3397.

[347] W. Loykowski, J. Kwiecinski, J.W. Wyrzkowski, T. Lada, Diffusionin Metals and Alloys-DIMETA-88, Diffusion and Defect Data-Solid State Data, Part A (Defect and Diffusion Forum) Balatonfured, Hungary 2 (1988) 957.

[348] K.L. Merkel, Interface Sci. 2 (1995) 311.

[349] M.I. Buckett, K.L. Merkle, Ultramicroscopy 56 (1994) 71.

[350] K.L. Merkel, in: W. Braue, R. Carpenter, U. Dahmen, M. Kim, T. Mitchell, R. Sinclair (Eds.), Atomic Structure and Chemistry of Boundaries/Interfaces, Tempe, AZ, 1997.

[351] R.D. Doherty, Kang Li, I. Samajdar, A.D. Rollett, J.P. Suni, R.T. Shuey, in: T.R. McNelley (Ed.), Proceedings of REX '96 The Third Inter Conference on Recrystallization and Related Phenomena, MIAS, Monterey, 1997, pp. 69-80.

[352] K.T. Aust, U. Erb, G. Palumbo, Mater. Sci. E. A176 (1994) 329.

[353] G. Avramovic-Cingara, K.T. Aust, D.D. Perovic, G. Palumbo, H.J. McQueen, Can. Metall. Quart. 34 (1995) 265.

[354] C. Cheung, U. Erb, G. Palumbo, Mater. Sci. E. A185 (1994) 39.

[355] L.C. Lim, T. Watanabe, Acta Metall. Mater. 38 (1990) 2507.

[356] G. Palumbo, P.J. King, K.T. Aust, U. Erb, P.C. Lichtenberger, Scr. Metall. Mater. 25 (1991) 1775.

[357] T. Watanabe, Text. Micros. 14 (1991) 739.

[358] M. Ferry, F.J. Humphreys, Acta Mater. 44 (1996) 3089.

[359] D.C. Crawford, G.S. Was, J. Electron Microsc. Tech. 19 (1991) 345.

[360] T. Watanabe, Mater. Sci. E. A166 (1993) 11.

[361] T. Watanabe, Scr. Metall. Mater. 27 (1992) 1497.

[362] C.B. Thompson, V. Randle, in: G.W. Bailey, J.M. Corbett, R.V.W. Dimlich, J.R. Michael, N.J. Zaluzec (Eds.), 1996 MSA Annual Meeting, San Francisco Press, MN, 1996, p. 356.

[363] T. Watanabe, Text. Micros. 20 (1993) 195.

[364] G.R. Canova, Y. Brechet, L.B. Kubin, B. Devincre, V. Pontikis, M. Condat, Solid Stat. Phen. 35-36 (1994) 101-106. 